\title{
Gepner type stability conditions on graded matrix factorizations
}

\author{
Yukinobu Toda
}

\begin{abstract}
We introduce the notion of Gepner type Bridgeland stability conditions on triangulated categories, which depends on a choice of an autoequivalence and a complex number. We conjecture the existence of Gepner type stability conditions on the triangulated categories of graded matrix factorizations of weighted homogeneous polynomials. Such a stability condition may give a natural stability condition for Landau-Ginzburg Bbranes, and correspond to the Gepner point of the stringy Kähler moduli space of a quintic threefold. The main result is to show our conjecture when the variety defined by the weighted homogeneous polynomial is a complete intersection of hyperplanes in a Calabi-Yau manifold with dimension less than or equal to two.
\end{abstract}

\section{Introduction}

\subsection{Motivation}

The Donaldson-Thomas (DT) invariants enumerate semi-stable coherent sheaves on Calabi-Yau threefolds, which have drawn much attention recently [Tho00]. We are interested in the following two problems in DT theory:

- Find constraints among DT invariants induced by autoequivalences of the derived category of coherent sheaves, for example Seidel-Thomas twists [ST01].

- Construct DT type invariants counting B-branes on Landau-Ginzburg (LG) models associated with a superpotential.

As for the former problem, there are several predictions in string theory on generating series of DT invariants, for example $S$-duality conjecture, the Ooguri-Strominger-Vafa conjecture [DM07], [OSV04]. There seem to be mysterious constraints among DT invariants behind such predictions, and we hope to reveal their origins via symmetries in the derived category. We believe that a key step toward this problem is to construct a Bridgeland stability condition on the derived category [Bri07] satisfying a certain symmetric property with respect to the given autoequivalence. For instance, the large volume limit (cf. Figure 1) for a quintic Calabi-Yau threefold $X$ is preserved by $\otimes \mathcal{O}_{X}(1)$, and there is an obvious constraint among DT invariants

Received 22 May 2013, accepted in final form 25 June 2014.

2010 Mathematics Subject Classification 14F05

Keywords: Bridgeland stability conditions, matrix factorizations

This journal is (C) Foundation Compositio Mathematica 2014. This article is distributed with Open Access under the terms of the Creative Commons Attribution Non-Commercial License, which permits non-commercial reuse, distribution, and reproduction in any medium, provided that the original work is properly cited. For commercial re-use, please contact the Foundation Compositio Mathematica.

This work is supported by World Premier International Research Center Initiative (WPI initiative), MEXT, Japan. This work is also supported by Grant-in Aid for Scientific Research grant (22684002) from the Ministry of Education, Culture, Sports, Science and Technology, Japan. 


\section{YUKINOBU TODA}

induced by $\otimes \mathcal{O}_{X}(1)$. Also a construction of a (weak) stability condition which is preserved under the derived dual, together with wall-crossing arguments [JS12], [KS08], play crucial roles in the proof of the rationality of the generating series of rank one DT type invariants counting curves [Bri11], [Tod10b], [Tod12].

As for the latter problem, in order to define the DT type invariants, we need to fix a stability condition for B-branes on LG models. A desired stability condition should be natural in some sense, so that it is an analogue of Gieseker stability on coherent sheaves. In mathematical terms, if the superpotential is given by a homogeneous polynomial $W$, the relevant B-brane category is Orlov's triangulated category of graded matrix factorizations $\operatorname{HMF}^{\mathrm{gr}}(W)$ [Orl09]. For instance, suppose that $W$ is the defining polynomial of a quintic Calabi-Yau threefold $X \subset \mathbb{P}^{4}$. Then a desired Bridgeland stability condition on $\operatorname{HMF}^{\mathrm{gr}}(W)$ may correspond to the Gepner point (cf. Figure 1) of the stringy Kähler moduli space of $X$, via mirror symmetry and the Orlov equivalence [Orl09]

$$
D^{b} \operatorname{Coh}(X) \stackrel{\sim}{\rightarrow} \operatorname{HMF}^{\mathrm{gr}}(W) .
$$

The Gepner point is an orbifold point in the stringy Kähler moduli space of $X$, with stabilizer group $\mathbb{Z} / 5 \mathbb{Z}$. Such an orbifold data may be translated into a certain symmetric property of the corresponding Bridgeland stability condition, which we focus on and pursue in this paper.

We have observed common keywords regarding the two problems above, namely a Bridgeland stability condition with a symmetric property. The motivating problem for this paper, which is rather ambitious and which we are not able to do at this moment, is to find a natural stability condition on $\operatorname{HMF}^{\mathrm{gr}}(W)$, and apply its symmetric property to obtain non-trivial constraints among DT invariants on $X$, via the Orlov equivalence (1) and wall-crossing arguments [JS12], [KS08] (cf. Subsection 1.5 (iii)).

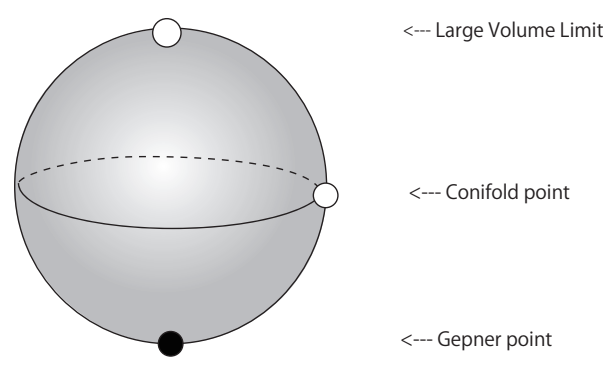

FIGURE 1. Stringy Kähler moduli space of a quintic threefold

\subsection{Gepner type stability conditions}

As we discussed, we are interested in constructing a natural stability condition on $\operatorname{HMF}^{\text {gr }}(W)$. So far there are few works on this problem, except [KST07], [Tak05], [Wa105], which will be discussed later. A serious issue is that, since the category $\operatorname{HMF}^{\mathrm{gr}}(W)$ is not a priori constructed as a derived category of some abelian category, it is not clear what 'natural' means. Our viewpoint is as follows: rather than constructing a stability condition in terms of graded matrix factorizations, we just extract and formulate the symmetric property of a desired stability condition, and try to find one satisfying such a property. We formulate it as a Gepner type property, which depends on a choice of a pair consisting of an autoequivalence and a complex number, given simply as follows. 


\section{GEPNER TYPE STABILITY CONDITIONS}

DeFinition 1.1. A stability condition $\sigma$ on a triangulated category $\mathcal{D}$ is called of Gepner type with respect to $(\Phi, \lambda) \in \operatorname{Aut}(\mathcal{D}) \times \mathbb{C}$ if the following condition holds:

$$
\Phi_{*} \sigma=\sigma \cdot(\lambda) \text {. }
$$

Obviously, any stability condition is of Gepner type with respect to $([k], k)$ for $k \in \mathbb{Z}$, where $[k]$ is the $k$-times composition of the shift functor [1]. On the other hand, there are several interesting examples in which the relation (2) holds with respect to non-trivial pairs $(\Phi, \lambda)$. As the name indicates, if $X \subset \mathbb{P}^{4}$ is a quintic threefold, a Gepner type stability condition on $D^{b} \operatorname{Coh}(X)$ with respect to the pair

$$
(\Phi, \lambda)=\left(\operatorname{ST}_{\mathcal{O}_{X}} \circ \otimes \mathcal{O}_{X}(1), \frac{2}{5}\right)
$$

conjecturally corresponds to the Gepner point, where $\mathrm{ST}_{\mathcal{O}_{X}}$ is the Seidel-Thomas twist [ST01] associated with $\mathcal{O}_{X}$. The fraction $2 / 5$ appears since the fivefold composition of the autoequivalence $\Phi$ becomes the twofold composition of the shift functor. This fact is best understood in terms of $\operatorname{HMF}^{\mathrm{gr}}(W)$ via the equivalence (1), as the autoequivalence $\Phi$ corresponds to the grade shift functor on the matrix factorization side.

At this moment, it seems to be a difficult problem to construct a stability condition on a quintic threefold corresponding to the Gepner point: we are not even able to construct a Bridgeland stability condition on a quintic threefold near the large volume limit point (cf. [BMT14]). However there are plenty of examples of weighted homogeneous polynomials, which are more amenable than quintic polynomials, but interesting enough to study. The goal of this paper is to construct Gepner type stability conditions on graded matrix factorizations in some of these interesting cases. In the quintic case, an attempt to construct a Gepner point leads to a conjectural stronger version of the Bogomolov-Gieseker inequality among Chern characters of stable sheaves on quintic threefolds. The details in this case will be discussed in the paper [Tod13].

\subsection{Gepner type stability conditions on graded matrix factorizations}

Let $W$ be a homogeneous element with degree $d$ in the weighted polynomial ring

$$
W \in A:=\mathbb{C}\left[x_{1}, x_{2}, \cdots, x_{n}\right], \quad \operatorname{deg} x_{i}=a_{i} \in \mathbb{Z}
$$

such that $(W=0) \subset \mathbb{C}^{n}$ has only an isolated singularity at the origin. The triangulated category of graded matrix factorizations of $W$, denoted by $\operatorname{HMF}^{\mathrm{gr}}(W)$, is defined to be the homotopy category of the dg category whose objects consist of data

$$
P^{0} \stackrel{p^{0}}{\rightarrow} P^{1} \stackrel{p^{1}}{\rightarrow} P^{0}(d)
$$

where $P^{i}$ are graded free $A$-modules of finite rank, $p^{i}$ are homomorphisms of graded $A$-modules, $P^{i} \mapsto P^{i}(1)$ is the shift of the grading, satisfying the following condition:

$$
p^{1} \circ p^{0}=\cdot W, \quad p^{0}(d) \circ p^{1}=\cdot W .
$$

In [Orl09], Orlov proved that the triangulated category $\operatorname{HMF}^{\mathrm{gr}}(W)$ is related to the derived category of coherent sheaves on the stack

$$
X:=(W=0) \subset \mathbb{P}\left(a_{1}, \cdots, a_{n}\right)
$$

depending on the sign of the Gorenstein index

$$
\varepsilon:=\sum_{i=1}^{n} a_{i}-d .
$$




\section{YUKINOBU TODA}

Let $\tau$ be the autoequivalence of $\operatorname{HMF}^{\mathrm{gr}}(W)$ induced by the grade shift functor $P^{\bullet} \mapsto P^{\bullet}(1)$. We propose the following conjecture on the existence of a Gepner type stability condition on $\operatorname{HMF}^{\mathrm{gr}}(W)$.

ConjeCture 1.2. There is a Gepner type stability condition

$$
\sigma_{G}=\left(Z_{G},\left\{\mathcal{P}_{G}(\phi)\right\}_{\phi \in \mathbb{R}}\right) \in \operatorname{Stab}\left(\operatorname{HMF}^{\text {gr }}(W)\right)
$$

with respect to $(\tau, 2 / d)$, whose central charge $Z_{G}$ is given by

$$
Z_{G}\left(P^{\bullet}\right)=\operatorname{str}\left(e^{2 \pi \sqrt{-1} / d}: P^{\bullet} \rightarrow P^{\bullet}\right) .
$$

Here the $e^{2 \pi \sqrt{-1} / d_{\text {-action }}} P^{\bullet}$ is induced by the $\mathbb{Z}$-grading on each $P^{i}$, and 'str' is the supertrace which respects the $\mathbb{Z} / 2 \mathbb{Z}$-grading on $P^{\bullet}$.

Similarly to the quintic case, the fraction $2 / d$ appears since the $d$-fold composition of $\tau$ coincides with [2]. We propose that, if there is a desired stability condition $\sigma_{G}$ in Conjecture 1.2, then it may be employed as a 'natural' stability condition for graded matrix factorizations. There are at least three reasons for this. Firstly, the Gepner type property with respect to $(\tau, 2 / d)$ resembles the following property of the classical Gieseker stability on coherent sheaves: for a polarized variety $(X, H)$, a coherent sheaf $E$ on $X$ is $H$-Gieseker semi-stable if and only if $E \otimes \mathcal{O}_{X}(H)$ is $H$-Gieseker semi-stable. In this sense, the desired stability condition $\sigma_{G}$ seems to be a natural analogue of the Gieseker stability on $\operatorname{Coh}(X)$ for graded matrix factorizations. Secondly, the Gepner type property with respect to $(\tau, 2 / d)$ turns out to be a very strong constraint for the stability conditions. Indeed, such a property characterizes the central charge $Z_{G}$ uniquely up to scalar multiplication (cf. Subsection 2.8). Also in some cases, we see that $\sigma_{G}$ is also unique up to shift (cf. Subsection 2.9) and one may expect that this holds in general. Thirdly, if there is such a $\sigma_{G}$, then the $\sigma_{G}$-semi-stable objects have a nice compatibility with the Serre functor on $\mathrm{HMF}^{\mathrm{gr}}(W)$. As a result, the moduli space of $\sigma_{G}$-semi-stable objects should have good local properties, for example smoothness, with a perfect obstruction theory, etc., depending on the given data $n, d, \varepsilon$ (cf. Subsection 1.5 (ii)). Therefore, whichever way $\sigma_{G}$ is constructed, it seems worth studying moduli spaces of $\sigma_{G}$-semi-stable objects, and the enumerative invariants defined by them.

\subsection{Result}

Before stating our result, we mention the earlier beautiful work by Takahashi [Tak05] and Kajiura-Saito-Takahashi [KST07]. They study the triangulated category $\operatorname{HMF}^{\text {gr }}(W)$ in the following cases: $n=a_{1}=1$ [Tak05] and $n=3, \varepsilon>0$ [KST07]. In these cases, they show that $\operatorname{HMF}^{\text {gr }}(W)$ is equivalent to the derived category of representations of a quiver of ADE type. As a result, $\mathrm{HMF}^{\mathrm{gr}}(W)$ has only a finite number of indecomposable objects up to shift, which are completely classified. Using such a classification, they construct a stability condition on $\operatorname{HMF}^{\text {gr }}(W)$ by the assignment of a phase for each indecomposable object. Their construction satisfies our Gepner type property, so Conjecture 1.2 is proved in these cases (cf. Subsection 2.7).

From our motivation, we are rather interested in cases where there are an infinite number of indecomposable objects up to shift, which are hard to classify, and form non-trivial moduli spaces. For instance if $\varepsilon \leqslant 0$, then the stack (4) is either Calabi-Yau or of general type, and it seems hopeless to construct $\sigma_{G}$ via classification of indecomposable objects. Our main result, formulated as follows, contains such cases (cf. Propositions 5.2, 5.4, 5.10, 5.18, 5.26). 


\section{GEPNER TYPE STABILITY CONDITIONS}

TABle 1. Possible types in Theorem 1.3

\begin{tabular}{rrrrrr}
\hline$n$ & $\varepsilon$ & $\left(a_{1}, \cdots, a_{n}\right)$ & $d$ & $W$ & $X$ \\
\hline 4 & 0 & $(1,1,1,1)$ & 4 & $x_{1}^{4}+x_{2}^{4}+x_{3}^{4}+x_{4}^{4}$ & K3 surface \\
4 & 0 & $(3,1,1,1)$ & 6 & $x_{1}^{2}+x_{2}^{6}+x_{3}^{6}+x_{4}^{6}$ & K3 surface \\
3 & -1 & $(1,1,1)$ & 4 & $x_{1}^{4}+x_{2}^{4}+x_{3}^{4}$ & genus 3 curve \\
3 & -1 & $(3,1,1)$ & 6 & $x_{1}^{2}+x_{2}^{6}+x_{3}^{6}$ & genus 2 curve \\
2 & -2 & $(1,1)$ & 4 & $x_{1}^{4}+x_{2}^{4}$ & 4 points \\
2 & -2 & $(3,1)$ & 6 & $x_{1}^{2}+x_{2}^{6}$ & 2 points \\
3 & 0 & $(1,1,1)$ & 3 & $x_{1}^{3}+x_{2}^{3}+x_{3}^{3}$ & elliptic curve \\
3 & 0 & $(2,1,1)$ & 4 & $x_{1}^{2}+x_{2}^{4}+x_{3}^{4}$ & elliptic curve \\
3 & 0 & $(3,2,1)$ & 6 & $x_{1}^{2}+x_{2}^{3}+x_{3}^{6}$ & elliptic curve \\
2 & -1 & $(1,1)$ & 3 & $x_{1}^{3}+x_{2}^{3}$ & 3 points \\
2 & -1 & $(2,1)$ & 4 & $x_{1}^{2}+x_{2}^{4}$ & 2 points \\
2 & -1 & $(3,2)$ & 6 & $x_{1}^{2}+x_{2}^{3}$ & 1 point \\
\hline
\end{tabular}

Theorem 1.3. Conjecture 1.2 is true if $n-4 \leqslant \varepsilon \leqslant 0$ and the stack $X$ defined by (4) does not contain stacky points.

The assumption that $X$ does not contain stacky points means that $X$ is indeed a smooth projective variety. The inequality $n-4 \leqslant \varepsilon \leqslant 0$ implies that $X$ is contained in a Calabi-Yau manifold of dimension less than or equal to two as a codimension $-\varepsilon$ complete intersection of hyperplanes. These conditions are restrictive, and the possible types $\left(a_{1}, \cdots, a_{n}, d, W, X\right)$ are completely classified. The classification with $n-\varepsilon=3,4$ and $W$ of Fermat type is given in Table 1.

Our strategy for proving Theorem 1.3 is as follows: by Orlov's theorem [Orl09], the condition $\varepsilon \leqslant 0$ allows us to describe $\operatorname{HMF}^{\text {gr }}(W)$ as a semi-orthogonal decomposition

$$
\operatorname{HMF}^{\mathrm{gr}}(W)=\left\langle\mathbb{C}(-1-\varepsilon), \cdots, \mathbb{C}(0), \Psi D^{b} \operatorname{Coh}(X)\right\rangle,
$$

where $\Psi$ is a fully faithful functor from $D^{b} \operatorname{Coh}(X)$ to $\operatorname{HMF}^{\text {gr }}(W)$, and $\mathbb{C}(i)$ is a certain exceptional object. We show that there is the heart of a bounded t-structure on $\operatorname{HMF}^{\mathrm{gr}}(W)$, given by the extension closure

$$
\mathcal{A}_{W}:=\langle\mathbb{C}(-1-\varepsilon), \cdots, \mathbb{C}(0), \Psi \operatorname{Coh}(X)\rangle_{\mathrm{ex}} .
$$

We describe the central charge $Z_{G}$ in terms of the generators of the heart $\mathcal{A}_{W}$, and take a suitable tilting $\mathcal{A}_{G}$ of $\mathcal{A}_{W}$ using the description of $Z_{G}$. We then show that the pair $\left(Z_{G}, \mathcal{A}_{G}\right)$ determines a stability condition $\sigma_{G}$ on $\operatorname{HMF}^{\mathrm{gr}}(W)$. It remains to show that $\sigma_{G}$ has a desired Gepner type property, and we reduce it to showing the $\sigma_{G}$-stability of certain objects in $\operatorname{HMF}^{\mathrm{gr}}(W)$.

We show that the construction above works in the situation of Theorem 1.3. However, unfortunately, several case by case arguments are involved in the proof, and that prevents us from constructing $\sigma_{G}$ beyond the cases in Theorem 1.3. For instance, they contain the proofs of the inequalities of numerical classes of certain objects in $\mathcal{A}_{W}$, which are required in proving the axiom of Bridgeland stability for $\left(Z_{G}, \mathcal{A}_{G}\right)$. They also contain checking the $\sigma_{G}$-stability of some objects in $\operatorname{HMF}^{\mathrm{gr}}(W)$, which we use to prove the Gepner type property of $\sigma_{G}$. The arguments above are in particular hard if the image of $Z_{G}$ is not discrete. In the situation of Theorem 1.3, fortunately, the image of $Z_{G}$ is always discrete and that makes our situation technically rather amenable. 


\section{YUKINOBU TODA}

For instance the image of $Z_{G}$ is not discrete if $d=5$, and the case of $\left(a_{1}, a_{2}, d\right)=(1,1,5)$ is not included in Table 1. Such a case will be discussed in the paper [Tod13], since it involves a subtle argument due to this non-discrete property.

In the list in Table 1 , the case $(n, \varepsilon)=(3,-1)$ seems to be the most interesting case, since we observe a new phenomenon relating graded matrix factorizations and coherent systems on the smooth projective curve $X$. Recall that a coherent system on $X$ consists of data

$$
V \otimes \mathcal{O}_{X} \rightarrow F
$$

where $F$ is a coherent sheaf on $X$ and $V$ is a finite dimensional $\mathbb{C}$-vector space. We show that the heart $\mathcal{A}_{W}$ is equivalent to the abelian category of coherent systems on $X$. In the construction of $\sigma_{G}$, we see that a Clifford type bound on the dimension of $V$ is involved. Such a Clifford type bound for semi-stable coherent systems was established by Lange-Newstead [LN08], and we apply their work. If $X$ has a higher dimension, the conjectural construction of $\sigma_{G}$ would predict a higher dimensional analogue of Clifford type bound for semi-stable coherent systems. In the case of a quintic surface, the details will be discussed in [Tod13].

\subsection{Future directions for research}

We believe that the work in this paper leads to several interesting directions for the future research. We discuss some of them.

(i) Descriptions of $\sigma_{G}$-semi-stable objects in terms of graded matrix factorizations. As we discussed in the previous subsection, our construction of $\sigma_{G}$ relies on Orlov's theorem, and it is not intrinsic in terms of graded matrix factorizations. It would be an interesting problem to see what kinds of graded matrix factorizations appear as $\sigma_{G}$-semi-stable objects, and compare them with the $R$-stability discussed in string theory [Wal05]. It may involve deeper understanding of Orlov equivalence, and we are not even able to give a mathematically rigorous candidate of the description of $\sigma_{G}$ purely in terms of graded matrix factorizations.

(ii) Constructing moduli spaces of $\sigma_{G}$-semi-stable graded matrix factorizations. It would be an important problem to construct moduli spaces of $\sigma_{G}$-semi-stable graded matrix factorizations, and study their properties. We expect that, using the argument of [Tod08], there exist Artin stacks of finite type

$$
\mathcal{M}_{G}^{\mathrm{s}}(\gamma) \subset \mathcal{M}_{G}^{\mathrm{ss}}(\gamma), \quad \gamma \in \mathrm{HH}_{0}(W)
$$

which parameterize $\sigma_{G^{-}}$(semi-)stable graded matrix factorizations $P^{\bullet}$ with $\operatorname{ch}\left(P^{\bullet}\right)=\gamma$. Here $\mathrm{HH}_{0}(W)$ is the zeroth Hochschild homology group of $\mathrm{HMF}^{\mathrm{gr}}(W)$, studied in [Dyc11], [PV11] and $[\mathrm{PV} 12]$. On the other hand, we have the following vanishing for $\left[P^{\bullet}\right] \in \mathcal{M}_{G}^{\mathrm{ss}}(\gamma)$ :

$$
\operatorname{Hom}^{i}\left(P^{\bullet}, P^{\bullet}\right)=0, \quad i>n-2-\frac{2 \varepsilon}{d} .
$$

This vanishing, which is proved in Lemma 2.18, is one of the important properties of Gepner type stability conditions. Since the space $\operatorname{Hom}^{i}\left(P^{\bullet}, P^{\bullet}\right)$ is responsible for the local deformation theory of $P^{\bullet}$, the moduli space $\mathcal{M}_{G}^{\text {ss }}(\gamma)$ would have good local properties depending on $n, d, \varepsilon$, for example it is smooth if $4>n-2 \varepsilon / d$, has a perfect obstruction theory if $5>n-2 \varepsilon / d$. Such properties would be important in constructing counting invariants of graded matrix factorizations, even in a non-CY3 situation.

(iii) DT type invariants counting $\sigma_{G}$-semi-stable graded matrix factorizations: Suppose that $\operatorname{HMF}^{\mathrm{gr}}(W)$ is a CY3 category. If Conjecture 1.2 is true, then (ii) would imply the 


\section{GEPNER TYPE STABILITY CONDITIONS}

existence of invariants

$$
\operatorname{DT}_{G}(\gamma) \in \mathbb{Q}, \quad \gamma \in \mathrm{HH}_{0}(W)
$$

which count $\sigma_{G^{-}}$-semi-stable matrix factorizations $P^{\bullet}$ with $\operatorname{ch}\left(P^{\bullet}\right)=\gamma$. These invariants may give a DT analogue of Fan-Jarvis-Ruan-Witten theory [FJR07] in Gromov-Witten theory. Also, the Gepner type property of $\sigma_{G}$ should yield an important identity

$$
\operatorname{DT}_{G}(\gamma)=\operatorname{DT}_{G}\left(\tau_{*} \gamma\right) .
$$

This identity, combined with Orlov's equivalence and wall-crossing argument [JS12], [KS08], may imply non-trivial constraints among the original sheaf counting DT invariants on $X$ induced by the autoequivalence $\mathrm{ST}_{\mathcal{O}_{X}} \circ \otimes \mathcal{O}_{X}(1)$. If this works, then it realizes an analogue of CalabiYau/Landau-Ginzburg correspondence in FJRW theory [CR10].

\subsection{Plan of the paper}

In Section 2, we introduce the notion of Gepner type stability conditions and propose a conjecture on the existence of Gepner type stability conditions on the triangulated category $\operatorname{HMF}^{\mathrm{gr}}(W)$. We also discuss some examples of our conjecture, and their uniqueness. In Section 3, we construct the heart $\mathcal{A}_{W}$ of a bounded t-structure on $\operatorname{HMF}^{\mathrm{gr}}(W)$, and describe it in terms of quiver representations or coherent systems. In Section 4, we explain how to compute the central charge in terms of generators of $\mathcal{A}_{W}$, and propose a general recipe for the construction of a Gepner type stability condition. In Section 5, we prove Theorem 1.3 by applying the strategy in Section 4 .

\section{Gepner type stability conditions}

In this section, we recall the definition of Bridgeland stability conditions on triangulated categories, group actions, and define the notion of Gepner type stability conditions. We then recall Orlov's triangulated categories of graded matrix factorizations, and discuss Gepner type stability conditions on them.

\subsection{Definitions}

Let $\mathcal{D}$ be a triangulated category and let $K(\mathcal{D})$ be its Grothendieck group. We first recall Bridgeland's definition of stability conditions on it.

Definition 2.1 [Bri07]. A stability condition $\sigma$ on $\mathcal{D}$ consists of a pair $\left(Z,\{\mathcal{P}(\phi)\}_{\phi \in \mathbb{R}}\right)$

$$
Z: K(\mathcal{D}) \rightarrow \mathbb{C}, \quad \mathcal{P}(\phi) \subset \mathcal{D}
$$

where $Z$ is a group homomorphism (called central charge) and $\mathcal{P}(\phi)$ is a full subcategory (called $\sigma$-semi-stable objects with phase $\phi$ ) satisfying the following conditions:

- For $0 \neq E \in \mathcal{P}(\phi)$, we have $Z(E) \in \mathbb{R}_{>0} \exp (\sqrt{-1} \pi \phi)$.

- For all $\phi \in \mathbb{R}$, we have $\mathcal{P}(\phi+1)=\mathcal{P}(\phi)[1]$.

- For $\phi_{1}>\phi_{2}$ and $E_{i} \in \mathcal{P}\left(\phi_{i}\right)$, we have $\operatorname{Hom}\left(E_{1}, E_{2}\right)=0$.

- For each $0 \neq E \in \mathcal{D}$, there is a collection of distinguished triangles

$$
E_{i-1} \rightarrow E_{i} \rightarrow F_{i} \rightarrow E_{i-1}[1], \quad E_{N}=E, E_{0}=0
$$

with $F_{i} \in \mathcal{P}\left(\phi_{i}\right)$ and $\phi_{1}>\phi_{2}>\cdots>\phi_{N}$.

The full subcategory $\mathcal{P}(\phi) \subset \mathcal{D}$ is shown to be an abelian category, and its simple objects are called $\sigma$-stable. In [Bri07], Bridgeland shows that there is a natural topology on the set of 


\section{YUKINOBU TODA}

'good' stability conditions $\operatorname{Stab}(\mathcal{D})$, and each of its connected components has the structure of a complex manifold.

Remark 2.2. The 'good' conditions above are called the 'numerical property' and the 'support property' in the literature. Although these properties are important in considering the space $\operatorname{Stab}(\mathcal{D})$, we omit the details since we focus on the construction of one specific stability condition.

Let $\operatorname{Aut}(\mathcal{D})$ be the group of autoequivalences on $\mathcal{D}$. There is a left $\operatorname{Aut}(\mathcal{D})$-action on the set of stability conditions on $\mathcal{D}$. For $\Phi \in \operatorname{Aut}(\mathcal{D})$, it acts on the pair (5) as follows:

$$
\Phi_{*}\left(Z,\{\mathcal{P}(\phi)\}_{\phi \in \mathbb{R}}\right)=\left(Z \circ \Phi^{-1},\{\Phi(\mathcal{P}(\phi))\}_{\phi \in \mathbb{R}}\right) .
$$

There is also a right $\mathbb{C}$-action on the set of stability conditions on $\mathcal{D}$. For $\lambda \in \mathbb{C}$, its acts on the pair (5) as follows:

$$
\left(Z,\{\mathcal{P}(\phi)\}_{\phi \in \mathbb{R}}\right) \cdot(\lambda)=\left(e^{-\sqrt{-1} \pi \lambda} Z,\{\mathcal{P}(\phi+\operatorname{Re} \lambda)\}_{\phi \in \mathbb{R}}\right) .
$$

The notion of Gepner type stability conditions is defined in terms of these group actions.

Definition 2.3. A stability condition $\sigma$ on a triangulated category $\mathcal{D}$ is called of Gepner type with respect to $(\Phi, \lambda) \in \operatorname{Aut}(\mathcal{D}) \times \mathbb{C}$ if the following condition holds:

$$
\Phi_{*} \sigma=\sigma \cdot(\lambda) .
$$

Here we give some trivial examples:

Example 2.4. (i) For $k \in \mathbb{Z}$, any stability condition $\sigma$ on a triangulated category $\mathcal{D}$ is of Gepner type with respect to $([k], k)$.

(ii) Let $\mathcal{A}$ be an abelian category and let $Z$ be a group homomorphism

$$
Z: K(\mathcal{A}) \rightarrow \mathbb{C}
$$

such that $Z(\mathcal{A} \backslash\{0\})$ is contained in $\mathbb{R}_{>0} e^{\sqrt{-1} \pi \theta}$ for some $\theta \in \mathbb{R}$. We set $\mathcal{P}(\phi)$ for $\phi \in[\theta, \theta+1)$ to be

$$
\mathcal{P}(\theta)=\mathcal{A}, \quad \mathcal{P}(\phi)=\{0\} \text { if } \phi \in(\theta, \theta+1) .
$$

Other $\mathcal{P}(\phi)$ are determined by the rule $\mathcal{P}(\phi+1)=\mathcal{P}(\phi)[1]$. Let $\Phi$ be an autoequivalence of $D^{b}(\mathcal{A})$ which preserves $\mathcal{A}$ and $Z$. Then the pair $\left(Z,\{\mathcal{P}(\phi)\}_{\phi \in \mathbb{R}}\right)$ is a Gepner type stability condition on $D^{b}(\mathcal{A})$ with respect to $(\Phi, 0)$.

Remark 2.5. A Gepner type stability conditions as in Example (ii) appears at a point in the space of stability conditions on $X=\omega_{\mathbb{P}^{2}}$ studied by [BM11]. Such a point corresponds to the orbifold point in the stringy Kähler moduli space of $X$, and $\mathcal{A}$ is the abelian category of representations of a McKay quiver. The autoequivalence $\Phi$ is given by

$$
\Phi=\mathrm{ST}_{\mathcal{O}^{2}} \circ \otimes \mathcal{O}_{X}(1)
$$

which induces the automorphism of the McKay quiver.

As we will see, there will be more interesting examples of Gepner type stability conditions on triangulated categories of graded matrix factorization.

\subsection{Triangulated categories of graded matrix factorizations}

Here we recall Orlov's construction of triangulated categories of graded matrix factorizations [Or109]. Let $A$ be a weighted graded polynomial ring

$$
A:=\mathbb{C}\left[x_{1}, x_{2}, \cdots, x_{n}\right], \quad \operatorname{deg} x_{i}=a_{i}
$$




\section{GePNER TyPE STABILITy CONDITIONS}

and let $W \in A$ be a homogeneous element of degree $d$. Throughout this paper, we always assume that $a_{1} \geqslant a_{2} \geqslant \cdots \geqslant a_{n}$, and $(W=0) \subset \mathbb{C}^{n}$ has an isolated singularity at the origin. For a graded $A$-module $P$, we denote by $P_{i}$ its degree $i$-part, and let $P(k)$ be the graded $A$-module whose grade is shifted by $k$; that is, $P(k)_{i}=P_{i+k}$.

Definition 2.6. A graded matrix factorization of $W$ consists of data

$$
P^{0} \stackrel{p^{0}}{\rightarrow} P^{1} \stackrel{p^{1}}{\rightarrow} P^{0}(d)
$$

where $P^{i}$ are graded free $A$-modules of finite rank, $p^{i}$ are homomorphisms of graded $A$-modules, satisfying the following conditions:

$$
p^{1} \circ p^{0}=\cdot W, \quad p^{0}(d) \circ p^{1}=\cdot W .
$$

The category $\operatorname{HMF}^{\mathrm{gr}}(W)$ is defined to be the homotopy category of graded matrix factorizations of $W$. Its objects consist of data (8), and the set of morphisms are given by the commutative diagrams of graded $A$-modules

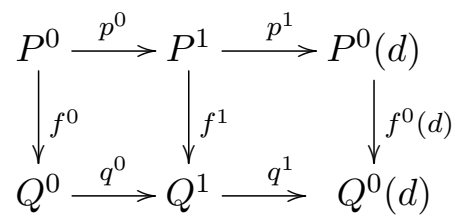

modulo null-homotopic morphisms: this diagram is null-homotopic if there are homomorphisms of graded $A$-modules

$$
h^{0}: P^{0} \rightarrow Q^{1}(-d), \quad h^{1}: P^{1} \rightarrow Q^{0}
$$

satisfying

$$
f^{0}=q^{1}(-d) \circ h^{0}+h^{1} \circ p^{0}, \quad f^{1}=q^{0} \circ h^{1}+h^{0}(d) \circ p^{1} .
$$

The category $\operatorname{HMF}^{\text {gr }}(W)$ has the structure of a triangulated category. The shift functor [1] sends data (8) to

$$
P^{1} \stackrel{-p^{1}}{\rightarrow} P^{0}(d) \stackrel{-p^{0}(d)}{\rightarrow} P^{1}(d)
$$

and the distinguished triangles are defined via the usual mapping cone constructions. The grade shift functor $P^{\bullet} \mapsto P^{\bullet}(1)$ induces the autoequivalence $\tau$ of $\operatorname{HMF}^{\text {gr }}(W)$ which satisfies the following identity:

$$
\tau^{\times d}=[2] .
$$

There is also a Serre functor $\mathcal{S}_{W}$ on $\operatorname{HMF}^{\mathrm{gr}}(W)$, described by $\tau, n$ and the Gorenstein index $\varepsilon$. The number $\varepsilon$ is defined as

$$
\varepsilon:=\sum_{i=1}^{n} a_{i}-d \in \mathbb{Z} .
$$

The Serre functor $\mathcal{S}_{W}$ on $\operatorname{HMF}^{\mathrm{gr}}(W)$ is given by (see, for instance, [KST09, Theorem 3.8])

$$
\mathcal{S}_{W}=\tau^{-\varepsilon}[n-2] \text {. }
$$

This description of the Serre functor will be used later in this paper.

\subsection{Relation to the triangulated categories of singularities}

The triangulated category $\operatorname{HMF}^{\mathrm{gr}}(W)$ is known to be equivalent to the derived category of singularities of the hypersurface singularity $(W=0) \subset \mathbb{C}^{n}$. This equivalence is often useful in 


\section{YUKINOBU TODA}

doing certain computations of matrix factorizations.

Let $R$ be the graded ring $R=A /(W)$ and let gr- $R$ be the abelian category of finitely generated graded $R$-modules. We denote by $D^{b}$ (grproj- $R$ ) the subcategory of $D^{b}$ (gr- $R$ ) consisting of perfect complexes of $R$-modules. The triangulated category of singularities is defined to be the quotient category

$$
D_{\mathrm{sg}}^{\mathrm{gr}}(R):=D^{b}(\mathrm{gr}-R) / D^{b}(\operatorname{grproj}-R) .
$$

The following result is proved in [Orl09].

TheOrem 2.7 [Or109, Theorem 3.10]. There is an equivalence of triangulated categories

$$
\text { Cok: } \operatorname{HMF}^{\mathrm{gr}}(W) \stackrel{\sim}{\rightarrow} D_{\mathrm{sg}}^{\mathrm{gr}}(R)
$$

sending a matrix factorization (8) to the cokernel of $p^{0}$.

The cokernel of $p^{0}$ is easily checked to be annihilated by $W$, so it is an $R$-module. Obviously, the equivalence (11) commutes with grade shift functors on both sides, so we use the same notation $\tau$ for the grade shift functor on $D_{\mathrm{sg}}^{\mathrm{gr}}(R)$.

Let

$$
m=\left(x_{1}, \cdots, x_{n}\right) \subset R
$$

be the maximal ideal and set the graded $R$-module $\mathbb{C}(j)$ to be $(R / m)(j)$. The graded $R$-module $\mathbb{C}(j)$ determines an object in $D_{\mathrm{sg}}^{\mathrm{gr}}(R)$. The matrix factorization given by

$$
\operatorname{Cok}^{-1}(\mathbb{C}(j)) \in \operatorname{HMF}^{\mathrm{gr}}(W)
$$

plays an important role. By [Dyc11, Corollary 2.7], it is given by the matrix factorization of the form

$$
\begin{aligned}
\bigoplus_{k \geqslant 0} \bigwedge^{2 k+1} m / m^{2} \otimes A(d k+j) & \stackrel{p^{0}}{\rightarrow} \bigoplus_{k \geqslant 0}^{2 k} \bigwedge^{2 k} m / m^{2} \otimes A(d k+j) \\
& \stackrel{p^{1}}{\rightarrow} \bigoplus_{k \geqslant 0} \bigwedge^{2 k+1} m / m^{2} \otimes A(d k+k+j) .
\end{aligned}
$$

We omit the descriptions of these morphisms $p^{0}, p^{1}$, as we will not use them. By an abuse of notation, we leave out $\mathrm{Cok}^{-1}$ and denote by $\mathbb{C}(j)$ the matrix factorization given by (13).

\subsection{Conjecture on the existence of Gepner type stability condition}

We are interested in constructing a natural stability condition on $\operatorname{HMF}^{\mathrm{gr}}(W)$. We require that such a stability condition be preserved under the grade shift functor $\tau$ in some sense. The following might be an analogous property for the classical $H$-Gieseker stability on a polarized variety $(X, H): H$-Gieseker semi-stable sheaves are preserved under $\otimes \mathcal{O}_{X}(H)$. Because of the identity (9), the expected stability condition is not exactly preserved by $\tau$ but the phases of semi-stable objects should be shifted by $2 / d$. This is nothing but the Gepner type property with respect to $(\tau, 2 / d)$.

There is a natural construction of a central charge $Z_{G}$ which might give a desired stability condition, and has already appeared in some articles [KST07], [Tak05], [Wal05]. For a graded matrix factorization (8), its image under $Z_{G}$ is symbolically defined by

$$
\operatorname{str}\left(e^{2 \pi \sqrt{-1} / d}: P^{\bullet} \rightarrow P^{\bullet}\right) .
$$




\section{Gepner type Stability CONDitions}

Here the $e^{2 \pi \sqrt{-1} / d}$-action is given by the $\mathbb{C}^{*}$-action on $P^{\bullet}$ induced by the $\mathbb{Z}$-grading on each $P^{i}$, and the ' $\mathrm{str}^{\prime}$ means the super trace of the $e^{2 \pi \sqrt{-1} / d}$-action which respects the $\mathbb{Z} / 2 \mathbb{Z}$-grading of $P^{\bullet}=P^{0} \oplus P^{1}$. More precisely, since $P^{i}$ are free $A$-modules of finite rank, they are written as

$$
P^{i} \cong \bigoplus_{j=1}^{m} A\left(n_{i, j}\right), \quad n_{i, j} \in \mathbb{Z} .
$$

Then (14) can be written as

$$
\sum_{j=1}^{m}\left(e^{2 n_{0, j} \pi \sqrt{-1} / d}-e^{2 n_{1, j} \pi \sqrt{-1} / d}\right) .
$$

Example 2.8. Let $\mathbb{C}(0)$ be the matrix factorization given by (13) for $j=0$. By the definition of $Z_{G}$, we have

$$
\begin{aligned}
Z_{G}(\mathbb{C}(0))= & \sum_{i=1}^{n} e^{-2 a_{i} \pi \sqrt{-1} / d}+\sum_{i_{1}<i_{2}<i_{3}} e^{-2\left(a_{i_{1}}+a_{i_{2}}+a_{i_{3}}\right) \pi \sqrt{-1} / d}+\cdots \\
& -1-\sum_{i_{1}<i_{2}} e^{-2\left(a_{i_{1}}+a_{i_{2}}\right) \pi \sqrt{-1} / d}-\sum_{i_{1}<i_{2}<i_{3}<i_{4}} \cdots \\
= & -\prod_{j=1}^{n}\left(1-e^{-2 a_{j} \pi \sqrt{-1} / d}\right) \neq 0 .
\end{aligned}
$$

It is easy to check that (14) descends to a group homomorphism

$$
Z_{G}: K\left(\operatorname{HMF}^{\mathrm{gr}}(W)\right) \rightarrow \mathbb{C} .
$$

Indeed, $Z_{G}$ is one of the components of the Chern character map of $\operatorname{HMF}^{\mathrm{gr}}(W)$ constructed in [PV12] (cf. Remark 2.16). As we stated in the introduction, we propose the following conjecture.

Conjecture 2.9. There is a Gepner type stability condition

$$
\sigma_{G}=\left(Z_{G},\left\{\mathcal{P}_{G}(\phi)\right\}_{\phi \in \mathbb{R}}\right) \in \operatorname{Stab}\left(\operatorname{HMF}^{\mathrm{gr}}(W)\right)
$$

with respect to $(\tau, 2 / d)$, where $Z_{G}$ is given by (14).

Note that the central charge $Z_{G}$ satisfies the condition (6) with respect to $(\tau, 2 / d)$ by construction. The problem is to construct full subcategories $\mathcal{P}_{G}(\phi) \subset \operatorname{HMF}^{\mathrm{gr}}(W)$ satisfying the desired property.

Remark 2.10. The image of $Z_{G}$ is contained in $\mathbb{Z}\left[e^{2 \pi \sqrt{-1} / d}\right]$, which may or may not be discrete depending on $d$. For instance, it is discrete if $d=3,4,6$, but not so if $d=5$.

\subsection{The case $n=1$}

As a toy example of Conjecture 2.9, let us consider the case $n=1$; that is,

$$
W=x^{d} \in \mathbb{C}[x], \quad \operatorname{deg} x=a .
$$

The case $a=1$ is worked out in [Tak05]. In this case, the triangulated category $\operatorname{HMF}^{\text {gr }}(W)$ is equivalent to the derived category of the path algebra of the Dynkin quiver of type $A_{d-1}$. As a result, we have a complete classification of indecomposable objects in $\operatorname{HMF}^{\mathrm{gr}}(W)$, given by

$$
Q_{j, l}:=\left\{A(j-l) \stackrel{x^{l}}{\rightarrow} A(j) \stackrel{x^{d-l}}{\rightarrow} A(j-l+d)\right\}
$$




\section{YUKINOBU TODA}

for $1 \leqslant l \leqslant d-1$ and $j \in \mathbb{Z}$. Note that $Q_{j, 1}$ coincides with $\mathbb{C}(j)$ given by $(13)$. We set $\phi\left(Q_{j, l}\right) \in \mathbb{Q}$ to be

$$
\phi\left(Q_{j, l}\right):=-\frac{1}{2}-\frac{l}{d}+\frac{2 j}{d} .
$$

For $\phi \in \mathbb{R}$, we define $\mathcal{P}_{G}(\phi) \subset \operatorname{HMF}^{\text {gr }}(W)$ to be the subcategory consisting of direct sums of objects $Q_{j, l}$ with $\phi\left(Q_{j, l}\right)=\phi$. Then Takahashi [Tak05] shows that the pair $\sigma_{G}=\left(Z_{G},\left\{\mathcal{P}_{G}(\phi)\right\}_{\phi \in \mathbb{R}}\right)$ is a desired stability condition in Conjecture 2.9. The case $a>1$ follows from the following lemma.

Lemma 2.11. Let $A$ be the graded ring (7), let $a \in \mathbb{Z}_{\geqslant 1}$ be the greatest common divisor of $\left(a_{1}, \cdots, a_{n}\right)$, and set $a_{i}^{\prime}=a_{i} / a$. Let $A^{\prime}$ be the graded ring defined by

$$
A^{\prime}=\mathbb{C}\left[x_{1}^{\prime}, x_{2}^{\prime}, \cdots, x_{n}^{\prime}\right], \quad \operatorname{deg} x_{i}^{\prime}=a_{i}^{\prime} .
$$

Given a homogeneous element $W \in A$ of degree $d$, we regard it as a homogeneous element $W^{\prime} \in A^{\prime}$ of degree $d^{\prime}=d / a$ by the identification $x_{i}=x_{i}^{\prime}$. Then if Conjecture 2.9 holds for $W^{\prime} \in A^{\prime}$, it also holds for $W \in A$.

Proof. There is an obvious fully-faithful functor of triangulated categories

$$
i: \operatorname{HMF}^{\mathrm{gr}}\left(W^{\prime}\right) \rightarrow \operatorname{HMF}^{\mathrm{gr}}(W)
$$

by multiplying each grading of $A^{\prime}$-modules which appears on the left-hand side by $a$. If $\tau^{\prime}$ is the grade shift on $\mathrm{HMF}^{\mathrm{gr}}\left(W^{\prime}\right)$ and $Z_{G}^{\prime}$ is the central charge (14) for $\operatorname{HMF}^{\mathrm{gr}}\left(W^{\prime}\right)$, we have

$$
\tau^{a} \circ i=i \circ \tau^{\prime}, \quad Z_{G} \circ i=Z_{G}^{\prime} .
$$

Also note that if there is a non-zero morphism of graded $A$-modules $A(m) \rightarrow A(n)$, then $m-n$ is divisible by $a$. This implies that $\operatorname{HMF}^{\mathrm{gr}}(W)$ has the following orthogonal decomposition:

$$
\left\langle i \mathrm{HMF}^{\mathrm{gr}}\left(W^{\prime}\right), \tau i \mathrm{HMF}^{\mathrm{gr}}\left(W^{\prime}\right), \cdots, \tau^{a-1} i \mathrm{HMF}^{\mathrm{gr}}\left(W^{\prime}\right)\right\rangle .
$$

Suppose that $\left(Z_{G}^{\prime},\left\{\mathcal{P}_{G}^{\prime}(\phi)\right\}_{\phi \in \mathbb{R}}\right)$ is a Gepner type stability condition on $\operatorname{HMF}^{\mathrm{gr}}\left(W^{\prime}\right)$ with respect to $\left(\tau^{\prime}, 2 / d^{\prime}\right)$. We set $\mathcal{P}_{G}(\phi)$ as follows:

$$
\mathcal{P}_{G}(\phi)=\left\{\bigoplus_{j=0}^{a-1} \tau^{j}\left(Q_{j}\right): Q_{j} \in i \mathcal{P}_{G}^{\prime}\left(\phi-\frac{2 j}{d}\right)\right\} .
$$

By (15) and (16), it is easy to check that $\left(Z_{G},\left\{\mathcal{P}_{G}(\phi)\right\}_{\phi \in \mathbb{R}}\right)$ is a Gepner type stability condition on $\operatorname{HMF}^{\text {gr }}(W)$ with respect to $(\tau, 2 / d)$.

Combining this with the argument for $n=a_{1}=1$, we obtain the following corollary.

Corollary 2.12. Conjecture 2.9 is true if $n=1$.

\subsection{Remarks for the case $n=2$}

We discuss Conjecture 2.9 in some more cases with small $n$. By Corollary 2.12, the next interesting case is $n=2$. In this case, the problem is trivial when $\varepsilon>0$. Indeed it is easy to check that

$$
\operatorname{HMF}^{\mathrm{gr}}(W)=\{0\}, \quad n=2, \varepsilon>0
$$

by using the equivalence (11). On the other hand, the triangulated category $\operatorname{HMF}^{\mathrm{gr}}(W)$ is nontrivial when $\varepsilon=0$. In this case, by applying a coordinate change if necessary, we may assume that

$$
W=x_{1} x_{2} \in \mathbb{C}\left[x_{1}, x_{2}\right]
$$




\section{GEPNER TYPE STABILITY CONDITIONS}

with $a_{1}$ and $a_{2}$ coprime by Lemma 2.11. Similarly to the case $n=1$, it turns out that there are only a finite number of indecomposable objects up to shift in $\operatorname{HMF}^{\mathrm{gr}}(W)$. They consist of the objects

$$
\mathbb{C}(j)[k], \quad 0 \leqslant j \leqslant d-1, \quad k \in \mathbb{Z}
$$

where $\mathbb{C}(j)$ is given by (13). Furthermore, the indecomposable objects are mutually orthogonal. This fact can be easily checked, for instance using Orlov's theorem [Or109], as given in Example 3.4 below. A desired stability condition $\sigma_{G}$ in Conjecture 2.9 is constructed as follows: we first choose $\phi_{0} \in \mathbb{R}$ so that $Z_{G}(\mathbb{C}(0)) \in \mathbb{R}_{>0} e^{2 \pi i \phi_{0}}$ and set

$$
\phi(\mathbb{C}(j)[k])=\phi_{0}+k+\frac{2 j}{a_{1}+a_{2}} .
$$

We define $\mathcal{P}_{G}(\phi)$ to be the subcategory consisting of direct sums of indecomposable objects with $\phi(*)=\phi$. Then by the argument above, $\sigma=\left(Z_{G},\left\{\mathcal{P}_{G}(\phi)\right\}_{\phi \in \mathbb{R}}\right)$ gives a desired stability condition. As a summary, we have the following.

Proposition 2.13. Conjecture 2.9 is true if $n=2$ and $\varepsilon \geqslant 0$.

The situation drastically changes when $n=2$ and $\varepsilon<0$. For instance, let us consider the case

$$
W=x_{1}^{d}+x_{2}^{d} \in \mathbb{C}\left[x_{1}, x_{2}\right], \quad \operatorname{deg} x_{i}=1, d \geqslant 3 .
$$

Even in such a simple case, Conjecture 2.9 does not seem obvious, and requires a deep understanding of the category $\operatorname{HMF}^{\mathrm{gr}}(W)$. This case is treated in this paper when $d \leqslant 4$. The $d=5$ case will be studied in [Tod13].

\subsection{Remarks for the case $n=3$}

Suppose $n=3$ and $\varepsilon>0$, the case studied by Kajiura-Saito-Takahashi [KST07]. In this case, $W$ is classified into the following ADE types [Sai87]:

$$
W\left(x_{1}, x_{2}, x_{3}\right)= \begin{cases}x_{1} x_{2}+x_{3}^{l+1} & A_{l}(l \geqslant 1) \\ x_{1}^{2}+x_{2}^{2} x_{3}+x_{3}^{l-1} & D_{l}(l \geqslant 4) \\ x_{1}^{2}+x_{2}^{3}+x_{3}^{4} & E_{6} \\ x_{1}^{2}+x_{2}^{3}+x_{2} x_{3}^{3} & E_{7} \\ x_{1}^{2}+x_{2}^{3}+x_{3}^{5} & E_{8} .\end{cases}
$$

Furthermore, the triangulated category $\operatorname{HMF}^{\mathrm{gr}}(W)$ is equivalent to the derived category of quiver representations of a Dynkin quiver of the corresponding ADE type. As a result, the category $\operatorname{HMF}^{\mathrm{gr}}(W)$ is shown to have only a finite number of indecomposable objects up to shift, which are completely classified. Similarly to the case $n=1$ (which is also interpreted as an $A_{l}$-case in the $n=3, \varepsilon>0$ list given above, by Knörrer periodicity [Knö87]) they assign phases to classified indecomposable objects, and prove the following result.

Theorem 2.14 [KST07, Theorem 4.2]. Conjecture 2.9 is true if $n=3$ and $\varepsilon>0$.

Conjecture 2.9 in the case $n=3$ and $\varepsilon \leqslant 0$ is not obvious, and a part of this case is treated later in this paper.

\subsection{Uniqueness of the central charge}

It is a natural question whether the Gepner type property uniquely characterizes $\sigma_{G}$ or not in some sense. For the central charge, it is true: $Z_{G}$ is characterized by the Gepner type property 


\section{YUKINOBU TODA}

with respect to $(\tau, 2 / d)$ up to scalar multiplication. Indeed we are only interested in central charges which factor through the Chern character map

$$
\text { ch: } \operatorname{HMF}^{\mathrm{gr}}(W) \rightarrow \mathrm{HH}_{0}(W) .
$$

The right-hand side is the Hochschild homology group of $\operatorname{HMF}^{\mathrm{gr}}(W)$, or more precisely of its dg enhancement. A general theory on Hochschild homology groups and Chern character maps on $\operatorname{HMF}^{\mathrm{gr}}(W)$ is available in [Dyc11], [PV11], [PV12].

Because $K\left(\mathrm{HMF}^{\mathrm{gr}}(W)\right)$ is not finitely generated in general, it would be more natural to define the set of central charges on $\operatorname{HMF}^{\mathrm{gr}}(W)$ as the dual space $\mathrm{HH}_{0}(W)^{\vee}$, rather than the original one in Definition 2.1. On the other hand, the autoequivalence $\tau$ on $\operatorname{HMF}^{\mathrm{gr}}(W)$ defines the linear isomorphism

$$
\tau_{*}: \mathrm{HH}_{*}(W) \stackrel{\cong}{\rightarrow} \mathrm{HH}_{*}(W) .
$$

This isomorphism induces the isomorphism $\tau_{*}^{\vee}$ on the space of central charges $\mathrm{HH}_{0}(W)^{\vee}$. The Gepner type property requires the central charge, regarded as an element in $\mathrm{HH}_{0}(W)^{\vee}$, to be an eigenvector with respect to $\tau_{*}^{\vee}$, with eigenvalue $e^{2 \pi \sqrt{-1} / d}$. The lemma below shows that such an eigenspace is one-dimensional.

Lemma 2.15. The eigenspace of the $\tau_{*}$-action on $\mathrm{HH}_{*}(W)$ with eigenvalue $e^{ \pm 2 \pi \sqrt{-1} / d}$ is onedimensional, and contained in $\mathrm{HH}_{0}(W)$.

Proof. We consider the $G:=\mu_{d}$-action on $\mathbb{C}^{n}$, given by

$$
e^{2 \pi \sqrt{-1} / d} \cdot\left(x_{1}, \cdots, x_{n}\right)=\left(e^{2 a_{1} \pi \sqrt{-1} / d} x_{1}, \cdots, e^{2 a_{n} \pi \sqrt{-1} / d} x_{n}\right) .
$$

By [PV11, Theorem 2.6.1 (i)], $\mathrm{HH}_{*}(W)$ is given by

$$
\mathrm{HH}_{*}(W) \cong \bigoplus_{\gamma \in G} \mathrm{H}\left(\mathbb{C}_{\gamma}^{n}, W_{\gamma}\right)^{G} .
$$

Here $H\left(\mathbb{C}^{n}, W\right)$ is defined by

$$
H\left(\mathbb{C}^{n}, W\right):=\left(\mathbb{C}\left[x_{1}, \cdots, x_{n}\right] /\left(\partial_{x_{1}} W, \cdots, \partial_{x_{n}} W\right)\right) d x_{1} \wedge \cdots \wedge d x_{n}
$$

and the space $\mathrm{H}\left(\mathbb{C}_{\gamma}^{n}, W_{\gamma}\right)$ is given by applying the construction above for

$$
\mathbb{C}_{\gamma}^{n}:=\left\{x \in \mathbb{C}^{n}: \gamma(x)=x\right\}, \quad W_{\gamma}:=\left.W\right|_{\mathbb{C}_{\gamma}^{n}} .
$$

Since $\tau^{\times d}=[2]$ on $\operatorname{HMF}^{\text {gr }}(W)$, we have $\tau_{*}^{\times d}=\mathrm{id}$ on $\mathrm{HH}_{*}(W)$. This implies that $\tau_{*}$ generates a $\mathbb{Z} / d \mathbb{Z}$-action on $\mathrm{HH}_{*}(W)$. By [PV11, Theorem 2.6.1 (ii)], decomposition (17) coincides with the character decomposition of $\mathrm{HH}_{*}(W)$ with respect to this $\mathbb{Z} / d \mathbb{Z}$-action. Therefore, noting that $\mathbb{C}_{e^{ \pm 2 \pi \sqrt{-1} / d}}^{n}=\{0\}$, the desired eigenspace is one dimensional by

$$
\mathrm{H}\left(\mathbb{C}_{e^{ \pm 2 \pi \sqrt{-1} / d}}^{n}, W_{e^{ \pm 2 \pi \sqrt{-1} / d}}\right) \cong \mathbb{C} .
$$

By the grading of $\mathrm{HH}_{*}(W)$ given in [PV11, Theorem 2.6.1], this eigenspace is contained in $\mathrm{HH}_{0}(W)$.

Remark 2.16. For $E \in \mathrm{HMF}^{\mathrm{gr}}(W)$, the $\gamma=e^{2 \pi \sqrt{-1} / d}$-component of $\operatorname{ch}(E)$ in the decomposition (17) coincides with the central charge $Z_{G}$ defined by (14) (cf. [PV12, Theorem 3.3.3]). In particular, $Z_{G}$ is given by an element in $\mathrm{HH}_{0}(W)^{\vee}$, which gives a basis of the eigenspace of $\tau_{*}^{\vee}$-action with eigenvalue $e^{2 \pi \sqrt{-1} / d}$.

Remark 2.17. Obviously, the set of Gepner type stability conditions with respect to $(\tau, 2 / d)$ is preserved under the natural right action of $\mathbb{C}$ on $\operatorname{Stab}\left(\operatorname{HMF}^{\mathrm{gr}}(W)\right)$. By Bridgeland's deformation 


\section{GePNER TyPE STABILITy CONDITIONS}

result [Bri07, Theorem 7.1], Lemma 2.15 implies that the set of such stability conditions forms a discrete subset in the quotient space $\operatorname{Stab}\left(\operatorname{HMF}^{\text {gr }}(W)\right) / \mathbb{C}$.

\subsection{Uniqueness of $\sigma_{G}$}

There are some cases in which not only $Z_{G}$ but also $\sigma_{G}$ is characterized by the Gepner type property. At least this is the case when all the indecomposable objects should become semistable. We first note the following lemma, which is an important property of Gepner type stability conditions.

Lemma 2.18. Suppose that $W \in A$ satisfies Conjecture 2.9 and $\sigma_{G}=\left(Z_{G},\left\{\mathcal{P}_{G}(\phi)\right\}_{\phi \in \mathbb{R}}\right)$ is a Gepner type stability condition with respect to $(\tau, 2 / d)$. For $\phi_{i} \in \mathbb{R}$ with $i=1,2$ and $k \in \mathbb{Z}$, suppose that the following inequality holds:

$$
\phi_{1}>\phi_{2}+n-k-2-\frac{2 \varepsilon}{d} .
$$

Then for any $F_{i} \in \mathcal{P}_{G}\left(\phi_{i}\right)$, we have $\operatorname{Hom}^{k}\left(F_{2}, F_{1}\right)=0$.

Proof. By the Serre functor given by (10), we have the isomorphism

$$
\operatorname{Hom}\left(F_{2}, F_{1}[k]\right) \cong \operatorname{Hom}\left(F_{1}, \tau^{-\varepsilon}\left(F_{2}\right)[n-k-2]\right)^{\vee} .
$$

By the Gepner type property with respect to $(\tau, 2 / d)$, we have

$$
\tau^{-\varepsilon}\left(F_{2}\right)[n-k-2] \in \mathcal{P}_{G}\left(\phi_{2}+n-k-2-\frac{2 \varepsilon}{d}\right) .
$$

By inequality (18), the phase of $F_{1}$ is bigger than that of this object, hence the right-hand side of (19) vanishes.

Using the above lemma, we show the following proposition:

Proposition 2.19. In the situation of Lemma 2.18, suppose that

$$
(n-3) d \leqslant 2 \varepsilon \text {. }
$$

Then all the other stability conditions satisfying the conditions in Conjecture 2.9 are obtained as $[2 m]_{*} \sigma_{G}$ for $m \in \mathbb{Z}$.

Proof. Let us take $F_{i} \in \mathcal{P}_{G}\left(\phi_{i}\right), i=1,2$ with $\phi_{1}>\phi_{2}$. Then Lemma 2.18 and assumption (20) show that $\operatorname{Hom}^{1}\left(F_{2}, F_{1}\right)=0$. This implies that any object in $\operatorname{HMF}^{\text {gr }}(W)$ whose HarderNarasimhan factors are $F_{1}, F_{2}$ decomposes into the direct sum of $F_{1}$ and $F_{2}$. Repeating this argument, any object $E \in \mathrm{HMF}^{\mathrm{gr}}(W)$ decomposes into the direct sum of $\sigma_{G}$-semi-stable objects. In particular, any non-zero indecomposable object $E \in \mathrm{HMF}^{\mathrm{gr}}(W)$ is $\sigma_{G}$-semi-stable. Its phase is denoted by $\phi_{E}$.

Let us fix a non-zero indecomposable object $M \in \operatorname{HMF}^{\mathrm{gr}}(W)$. By the result of [BFK12, Theorem 5.16], the objects $\tau^{i}(M), 0 \leqslant i \leqslant d-1$ generate the triangulated category $\mathrm{HMF}^{\mathrm{gr}}(W)$. Therefore for any non-zero indecomposable object $E \in \mathrm{HMF}^{\mathrm{gr}}(W)$, there are $0 \leqslant i \leqslant d-1$ and $j \in \mathbb{Z}$ such that $\operatorname{Hom}\left(E, \tau^{i}(M)[j]\right) \neq 0$. This implies that

$$
\phi_{E} \leqslant j+\phi_{M}+\frac{2 i}{d} \text {. }
$$

Also by the Serre functor $(10)$, we have $\operatorname{Hom}\left(\tau^{i}(M)[j], \mathcal{S}_{W}(E)\right) \neq 0$, which implies that

$$
j+\phi_{M}-n+2+\frac{2 i}{d}+\frac{2 \varepsilon}{d} \leqslant \phi_{E} .
$$




\section{YUKINOBU TODA}

Combining this with the assumption (20), we obtain

$$
\phi_{E} \in\left[j+\phi_{M}+\frac{2 i}{d}-1, j+\phi_{M}+\frac{2 i}{d}\right] .
$$

Now suppose that $\sigma_{G}^{\prime}$ is another stability condition which satisfies the condition in Conjecture 2.9. Then there is an $m \in \mathbb{Z}$ such that the phase of $M$ with respect to $[-2 m]_{*} \sigma_{G}^{\prime}$ coincides with $\phi_{M}$. If $\phi_{E}^{\prime}$ is the phase of the indecomposable object $E$ with respect to $[-2 m]_{*} \sigma_{G}^{\prime}$, then $\phi_{E}^{\prime}$ is also contained in the right-hand side of (21). Since both of the central charges of $\sigma_{G}$ and $[-2 m]_{*} \sigma_{G}^{\prime}$ are the same $Z_{G}$, it follows that $\phi_{E}^{\prime}=\phi_{E}$. Therefore $\sigma_{G}^{\prime}=[2 m]_{*} \sigma_{G}$ follows.

The proof of Proposition 2.19 immediately implies the following.

Corollary 2.20. In the situation of Proposition 2.19, there is a function $\phi(*)$ from the set of indecomposable objects in $\mathrm{HMF}^{\mathrm{gr}}(W)$ to real numbers such that $\mathcal{P}_{G}(\phi)$ consists of direct sums of indecomposable objects $E$ with $\phi(E)=\phi$.

Remark 2.21. Inequality (20) is satisfied in the cases of Corollary 2.12, Theorem 2.13 and Theorem 2.14. In the list in Table 1 , it is satisfied except for $(n, \varepsilon)=(4,0)$ and $(3,-1)$.

Remark 2.22. If we believe Conjecture 2.9, the proof of Proposition 2.19 predicts that $Z_{G}(E) \neq 0$ for any non-zero indecomposable object $E \in \operatorname{HMF}^{\mathrm{gr}}(W)$ as long as inequality (20) is satisfied. This seems to be not an obvious property of graded matrix factorizations.

\section{T-structures on triangulated categories of graded matrix factorizations}

In this section, we construct and study the hearts of bounded t-structures on $\operatorname{HMF}^{\mathrm{gr}}(W)$, via Orlov's theorem relating $\operatorname{HMF}^{\text {gr }}(W)$ with the derived category of coherent sheaves on $(W=0)$. In what follows, we use the same notation in the previous section.

\subsection{Orlov's theorem}

In [Orl09], Orlov proves his famous theorem relating the triangulated category $\operatorname{HMF}^{\mathrm{gr}}(W)$ to the derived category of coherent sheaves on the Deligne-Mumford stack

$$
X:=(W=0) \subset \mathbb{P}\left(a_{1}, \cdots, a_{n}\right) .
$$

Using the notation in Subsection 2.3, Orlov's theorem is stated in the following way.

Theorem 3.1 [Orl09, Theorem 2.5]. (i) If $\varepsilon>0$, there is a fully faithful functor

$$
\Phi_{i}: \operatorname{HMF}^{\mathrm{gr}}(W) \hookrightarrow D^{b} \operatorname{Coh}(X)
$$

such that we have the semi-orthogonal decomposition

$$
D^{b} \operatorname{Coh}(X)=\left\langle\mathcal{O}_{X}(-i-\varepsilon+1), \cdots, \mathcal{O}_{X}(-i), \Phi_{i} \operatorname{HMF}^{\mathrm{gr}}(W)\right\rangle .
$$

(ii) If $\varepsilon \leqslant 0$, there is a fully faithful functor

$$
\Psi_{i}: D^{b} \operatorname{Coh}(X) \hookrightarrow \operatorname{HMF}^{\mathrm{gr}}(W)
$$

such that we have the semi-orthogonal decomposition

$$
\operatorname{HMF}^{\mathrm{gr}}(W)=\left\langle\mathbb{C}(-i-\varepsilon), \cdots, \mathbb{C}(-i+1), \Psi_{i} D^{b} \operatorname{Coh}(X)\right\rangle .
$$

In particular, $\Psi_{i}$ is an equivalence if $\varepsilon=0$. 


\section{GEPNER TYPE STABILITY CONDITIONS}

In this paper we deal with the case $\varepsilon \leqslant 0$, so we only explain the construction of $\Psi_{i}$. It is the composition of the following functors:

$$
\Psi_{i}: D^{b} \operatorname{Coh}(X) \stackrel{\mathbf{R} \omega_{i}}{\rightarrow} D^{b}(\mathrm{gr}-R) \stackrel{\pi}{\rightarrow} D_{\mathrm{sg}}^{\mathrm{gr}}(R) \stackrel{\mathrm{Cok}^{-1}}{\rightarrow} \operatorname{HMF}^{\mathrm{gr}}(W),
$$

where $\mathrm{Cok}^{-1}$ is the inverse of (11), $\pi$ is the natural projection and $\mathbf{R} \omega_{i}$ is defined by

$$
\mathbf{R} \omega_{i}(E):=\bigoplus_{j \geqslant i} \mathbf{R} \operatorname{Hom}_{\mathcal{O}_{X}}\left(\mathcal{O}_{X}, E(j)\right) .
$$

The functor $\Psi_{i}$ is not compatible with grade shift functors. If $\varepsilon=0$, that is, $X$ is a Calabi-Yau stack, then their difference is described by a Seidel-Thomas twist functor [ST01] on $D^{b} \operatorname{Coh}(X)$

$$
\mathrm{ST}_{E}(*):=\text { Cone }\left(\mathbf{R} \operatorname{Hom}_{\mathcal{O}_{X}}(E, *) \otimes E \rightarrow *\right)
$$

for a spherical object $E \in D^{b} \operatorname{Coh}(X)$, for example a line bundle. The following result is suggested by Kontsevich and proved in [BFK12].

Proposition 3.2 [BFK12, Proposition 5.8]. If $\varepsilon=0$, the following diagram commutes:

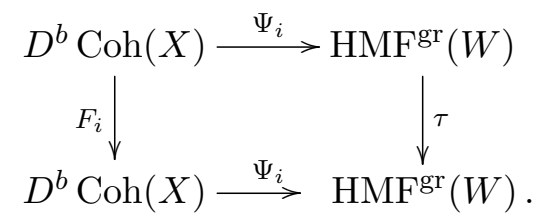

Here $F_{i}:=\mathrm{ST}_{\mathcal{O}_{X}(-i+1)} \circ \otimes \mathcal{O}_{X}(1)$.

Remark 3.3. In [BFK12, Proposition 5.8], a comparison result similar to Proposition 3.2 is also obtained for $\varepsilon \neq 0$. We only mention the case $\varepsilon=0$ since we only use the result in this case.

Example 3.4. Let us consider the case $n=2$ and $\varepsilon=0$. In the same situation as in Subsection 2.6, we have

$$
X \cong\left[\mathrm{pt} / \mathbb{Z}_{a_{1}}\right] \coprod\left[\mathrm{pt} / \mathbb{Z}_{a_{2}}\right]
$$

Here $\mathbb{Z}_{a_{i}}:=\mathbb{Z} / a_{i} \mathbb{Z}$ acts on the smooth one-point pt trivially, and $[* / *]$ means the quotient stack. Therefore we have the orthogonal decomposition

$$
D^{b} \operatorname{Coh}(X)=\left\langle V_{1}^{0}, \cdots, V_{1}^{a_{1}-1}, V_{2}^{0}, \cdots, V_{2}^{a_{2}-1}\right\rangle
$$

for one-dimensional $\mathbb{Z}_{a_{i}}$-representations $V_{i}^{j}$ with weight $j$. By Proposition 3.2 , the equivalence $\Psi_{1}$ identifies $\tau$ on $\operatorname{HMF}^{\mathrm{gr}}(W)$ with $F_{1}=\mathrm{ST}_{\mathcal{O}_{X}} \circ \otimes \mathcal{O}_{X}(1)$. The equivalence $F_{1}$ transforms $V_{i}^{j}$ in the following way:

$$
\begin{aligned}
V_{1}^{0} & \mapsto V_{1}^{1} \mapsto \cdots \mapsto V_{1}^{a_{1}-1} \mapsto \\
& \mapsto V_{2}^{0}[1] \mapsto \cdots \mapsto V_{2}^{a_{2}-1}[1] \mapsto V_{1}^{0}[2]
\end{aligned}
$$

In particular, $\mathrm{HMF}^{\mathrm{gr}}(W)$ has the description stated in Subsection 2.6.

\subsection{Construction of t-structures}

In this subsection, we construct the hearts of bounded t-structures on $\operatorname{HMF}^{\mathrm{gr}}(W)$ when $\varepsilon \leqslant 0$. We introduce the following notation: for a triangulated category $\mathcal{D}$ and a set of objects $\mathcal{S} \subset \mathcal{D}$, we denote by $\langle\mathcal{S}\rangle_{\text {ex }}$ the extension closure of $\mathcal{S}$; that is, the smallest extension-closed subcategory of $\mathcal{D}$ which contains objects in $\mathcal{S}$. The constructions of our hearts are based on the semi-orthogonal decomposition (23) and the following well-known fact. 


\section{YUKINOBU TODA}

Lemma 3.5. Let $\mathcal{D}$ be a triangulated category and let

$$
\mathcal{D}=\left\langle\mathcal{D}_{N}, \cdots, \mathcal{D}_{2}, \mathcal{D}_{1}\right\rangle
$$

be a semi-orthogonal decomposition. Suppose that $\mathcal{C}_{i} \subset \mathcal{D}_{i}$ are hearts of bounded t-structures satisfying $\operatorname{Hom}^{\leqslant 0}\left(\mathcal{C}_{j}, \mathcal{C}_{i}\right)=0$ for $j>i$. Then there is a bounded t-structure on $\mathcal{D}$ whose heart $\mathcal{C}$ is given by $\left\langle\mathcal{C}_{i}: 1 \leqslant i \leqslant N\right\rangle_{\mathrm{ex}}$.

Proof. The result is obviously reduced to the case $N=2$, which is proved in [CP10, Lemma 2.1].

Remark 3.6. In the situation of Lemma 3.5, any object $E \in \mathcal{C}$ admits a filtration

$$
0=E_{0} \subset E_{1} \subset \cdots \subset E_{N}=E
$$

such that $E_{i} / E_{i-1}$ is an object in $\mathcal{C}_{i}$.

Remark 3.7. If the abelian category $\mathcal{C}_{i}$ is generated by an exceptional object $F_{i} \in \mathcal{C}_{i}$, then the heart $\mathcal{C}$ is the extension closure $\left\langle F_{N}, \cdots, F_{1}\right\rangle_{\text {ex }}$. In this case, the sequence $\left(F_{N}, \cdots, F_{2}, F_{1}\right)$ is called an ext-exceptional collection.

We have the following proposition.

Proposition 3.8. Suppose that $\varepsilon \leqslant 0$. For each $i \in \mathbb{Z}$, there is a bounded t-structure on $\mathrm{HMF}^{\mathrm{gr}}(W)$ whose heart $\mathcal{A}_{i}$ is given by

$$
\mathcal{A}_{i}=\left\langle\mathbb{C}(-i-\varepsilon), \cdots, \mathbb{C}(-i+1), \Psi_{i} \operatorname{Coh}(X)\right\rangle_{\mathrm{ex}} .
$$

Proof. By following Orlov's argument in [Or109, Theorem 2.5], we see the following: there is an admissible subcategory $\mathcal{T}_{i} \subset D^{b}$ (gr- $R$ ) with a semi-orthogonal decomposition

$$
\mathcal{T}_{i}=\left\langle\mathbb{C}(-i-\varepsilon), \cdots, \mathbb{C}(-i+1), \mathbf{R} \omega_{i} D^{b} \operatorname{Coh}(X)\right\rangle
$$

and an equivalence

$$
\mathcal{T}_{i} \stackrel{\sim}{\rightarrow} \operatorname{HMF}^{\mathrm{gr}}(W)
$$

which identifies the semi-orthogonal decomposition (26) with the right-hand side of (23). Here $\mathbf{R} \omega_{i}$ is the functor defined by (25). Therefore by Lemma 3.5, it is enough to show that

$$
\begin{aligned}
& \operatorname{Hom}_{\mathrm{gr}-R}^{\leqslant 0}\left(\mathbb{C}(j), \mathbb{C}\left(j^{\prime}\right)\right)=0 \\
& \operatorname{Hom}_{\mathrm{gr}-R}^{\leqslant 0}\left(\mathbb{C}(j), \mathbf{R} \omega_{i} \operatorname{Coh}(X)\right)=0
\end{aligned}
$$

for $j, j^{\prime} \in[-i+1,-i-\varepsilon]$ with $j^{\prime}<j$. Assertion (27) is obvious since $\mathbb{C}(j)$ is a simple object in the heart gr- $R \subset D^{b}(\mathrm{gr}-R)$. As for assertion (28), since we have $\mathbf{R} \omega_{i}(F) \in D^{\geqslant 0}$ (gr- $R$ ) for $F \in \operatorname{Coh}(X)$, it follows that

$$
\operatorname{Hom}_{\mathrm{gr}-R}^{\leqslant 0}\left(\mathbb{C}(j), \mathbf{R} \omega_{i}(F)\right) \cong \operatorname{Hom}_{\mathrm{gr}-R}^{\leqslant 0}\left(\mathbb{C}(j), \omega_{i}(F)\right) .
$$

Here $\omega_{i}(F) \in$ gr- $R$ is the zeroth cohomology of $\mathbf{R} \omega_{i}(F)$. Since $\omega_{i}(F) \in$ gr- $R$ is concentrated in the degree $\geqslant i$ parts, and $\mathbb{C}(j)$ is in the degree $<i$ parts, the vector space (29) vanishes.

In what follows, we always assume that $\varepsilon \leqslant 0$. We only focus on the case $i=1$ in the proposition above.

Definition 3.9. Suppose that $\varepsilon \leqslant 0$. We define $\mathcal{A}_{W}:=\mathcal{A}_{1}$ and $\Psi:=\Psi_{1}$; that is,

$$
\mathcal{A}_{W}=\langle\mathbb{C}(-1-\varepsilon), \cdots, \mathbb{C}(0), \Psi \operatorname{Coh}(X)\rangle_{\mathrm{ex}} .
$$




\section{GePNer TyPE STABILITY CONDITIONS}

\subsection{Description of certain objects in $\mathcal{A}_{W}$}

This subsection is devoted to investigating some objects in $\mathcal{A}_{W}$ which will be used later. By definition, we call a closed point $x \in X$ stacky if the stabilizer group at $x$ is non-trivial. Let us describe $\Psi\left(\mathcal{O}_{x}\right)$ for a non-stacky point $x \in X$. Note that $X$ is a closed substack

$$
X \subset\left[\left(\mathbb{C}^{n} \backslash\{0\}\right) / \mathbb{C}^{*}\right],
$$

where $\mathbb{C}^{*}$ acts on $\mathbb{C}^{n}$ via weight $\left(a_{1}, \cdots, a_{n}\right)$. Hence $x \in X$ is represented by a point $\left(p_{1}, \cdots, p_{n}\right) \in$ $\mathbb{C}^{n}$. We define the graded $R$-module $M(x)$ to be

$$
M(x):=\bigoplus_{j \geqslant 1} \mathbb{C} e_{j},
$$

where $e_{j}$ is concentrated in degree $j$, and the action of $x_{i}$ sends $e_{j}$ to $p_{i} e_{j+a_{i}}$. Obviously, if $x \in X$ is non-stacky, then $\mathbf{R} \omega_{1}\left(\mathcal{O}_{x}\right)$ is a graded $R$-module and isomorphic to $M(x)$. The object $\Psi\left(\mathcal{O}_{x}\right)$ is obtained by applying the inverse $\mathrm{Cok}^{-1}$ of (11) to $M(x)$, after regarding it as an object in $D_{\mathrm{sg}}^{\mathrm{gr}}(R)$. Using this description, we have the following lemma.

Lemma 3.10. For any non-stacky point $x \in X$, we have the exact sequence in $\mathcal{A}_{W}$

$$
0 \rightarrow \Psi\left(\mathcal{O}_{x}\right) \rightarrow \tau \Psi\left(\mathcal{O}_{x}\right) \rightarrow \mathbb{C}(0) \rightarrow 0 .
$$

Proof. The result obviously follows from the following exact sequence as graded $R$-modules

$$
0 \rightarrow M(x) \rightarrow M(x)(1) \rightarrow \mathbb{C}(0) \rightarrow 0 .
$$

Next, let us consider the following object

$$
\mathbb{C}(-\varepsilon) \in \operatorname{HMF}^{\mathrm{gr}}(W) .
$$

This object is described in terms of the generators of $\mathcal{A}_{W}$. We have the following lemma.

Lemma 3.11. We have $\mathbb{C}(-\varepsilon)[-1] \in \mathcal{A}_{W}$. Furthermore, there is a filtration in $\mathcal{A}_{W}$

$$
0 \subset E_{-1} \subset E_{0} \subset \cdots \subset E_{-1-\varepsilon}=\mathbb{C}(-\varepsilon)[-1]
$$

such that the following holds for $0 \leqslant i \leqslant-1-\varepsilon$

$$
E_{-1} \cong \Psi\left(\omega_{X}\right), \quad E_{i} / E_{i-1} \cong \mathbb{C}(i) \otimes R_{-i-\varepsilon} .
$$

Proof. Let $m \subset R$ be the maximal ideal (12). We have the exact sequence

$$
0 \rightarrow m(-\varepsilon) \rightarrow R(-\varepsilon) \rightarrow \mathbb{C}(-\varepsilon) \rightarrow 0
$$

in gr- $R$ which implies $\mathbb{C}(-\varepsilon)[-1] \cong m(-\varepsilon)$ in $D_{\mathrm{sg}}^{\mathrm{gr}}(R)$. Let $m_{\geqslant i} \subset m$ be the ideal generated by monomials with degree greater than or equal to $i$. We have the following filtration in gr- $R$ :

$$
m_{\geqslant 1-\varepsilon} \subset m_{\geqslant-\varepsilon} \subset \cdots \subset m_{\geqslant 2} \subset m_{\geqslant 1}=m
$$

such that the following holds:

$$
\left(m_{\geqslant j} / m_{\geqslant j+1}\right)(-\varepsilon) \cong \mathbb{C}(-j-\varepsilon) \otimes R_{j}, \quad 1 \leqslant j \leqslant-\varepsilon .
$$

Therefore it is enough to show that $m_{\geqslant 1-\varepsilon}(-\varepsilon)$ is isomorphic to $\mathbf{R} \omega_{1}\left(\omega_{X}\right)$ in gr- $R$. Since $\omega_{X} \cong$ $\mathcal{O}_{X}(-\varepsilon)$ and $H^{k}\left(X, \omega_{X}(j)\right) \cong 0$ for $k \neq 0$ and $j \geqslant 1$, we have

$$
\mathbf{R} \omega_{1}\left(\omega_{X}\right) \cong \bigoplus_{j \geqslant 1} H^{0}\left(X, \mathcal{O}_{X}(-\varepsilon+j)\right) .
$$

Obviously, the right-hand side is isomorphic to $m_{\geqslant 1-\varepsilon}(-\varepsilon)$ as a graded $R$-module. 


\section{YuKINOBU TODA}

The lemma above can be applied to carry out computations on the left adjoint of $\Psi$, denoted by

$$
\Psi^{L}: \operatorname{HMF}^{\mathrm{gr}}(W) \rightarrow D^{b} \mathrm{Coh}(X) .
$$

We have the following lemma.

Lemma 3.12. The object $\Psi^{L}(\mathbb{C}(0))$ is isomorphic to $\mathcal{O}_{X}[1]$.

Proof. Let $\Psi^{R}$ be the right adjoint of $\Psi$. Note that $\Psi^{L}$ and $\Psi^{R}$ are related by

$$
\Psi^{L}=\mathcal{S}_{X}^{-1} \circ \Psi^{R} \circ \mathcal{S}_{W}
$$

where $\mathcal{S}_{X}=\otimes \omega_{X}[n-2]$ is the Serre functor of $D^{b} \operatorname{Coh}(X)$. By (10), we have

$$
\begin{aligned}
\Psi^{L}(\mathbb{C}(0)) & \cong \mathcal{S}_{X}^{-1} \circ \Psi^{R}(\mathbb{C}(-\varepsilon))[n-2] \\
& \cong \mathcal{S}_{X}^{-1}\left(\omega_{X}[1]\right)[n-2] \\
& \cong \mathcal{O}_{X}[1] .
\end{aligned}
$$

Here the second isomorphism follows from Lemma 3.11.

Remark 3.13. By this lemma, it follows that

$$
\operatorname{Hom}_{\operatorname{HMF}^{\mathrm{gr}}(W)}^{1}\left(\mathbb{C}(0), \Psi\left(\mathcal{O}_{x}\right)\right) \cong \operatorname{Hom}_{X}\left(\mathcal{O}_{X}, \mathcal{O}_{x}\right),
$$

which is one-dimensional for $x \in X$. Since $\tau \Psi\left(\mathcal{O}_{x}\right)$ is indecomposable, the exact sequence (32) is a unique non-trivial extension.

Remark 3.14. For $F \in \operatorname{Coh}(X)$, suppose that $\mathbf{R} \omega_{1}(F)$ is a graded $R$-module. Then Lemma 3.12 and the argument in Proposition 3.8 imply

$$
\operatorname{Ext}_{\mathrm{gr}-R}^{1}\left(\mathbb{C}(0), \mathbf{R} \omega_{1}(F)\right) \cong H^{0}(X, F) .
$$

For $u \in H^{0}(X, F)$, we have the corresponding extension in gr- $R$

$$
0 \rightarrow \mathbf{R} \omega_{1}(F) \rightarrow M_{u} \rightarrow \mathbb{C}(0) \rightarrow 0 .
$$

The graded $R$-module $M_{u}$ is described in the following way: as a graded $\mathbb{C}$-vector space, it is the direct sum $\mathbb{C}(0) \oplus \mathbf{R} \omega_{1}(F)$, and the action of $x_{i} \in R$ sends $1 \in \mathbb{C}(0)$ to $u \cdot x_{i} \in H^{0}(X, F(1))$.

\subsection{Description of $\mathcal{A}_{W}$ via quiver representations}

In this subsection, we assume that $n=2$ and describe the heart $\mathcal{A}_{W}$ in terms of certain quiver representations. In this case, $X$ is a smooth zero-dimensional Deligne-Mumford stack, and $\operatorname{Coh}(X)$ is generated by mutually orthogonal exceptional objects. Therefore by (30), the abelian category $\mathcal{A}_{W}$ is the extension closure of an ext-exceptional collection. We first compute other Hom groups between these exceptional objects, and then describe $\mathcal{A}_{W}$ via the ext-quivers with relations.

For a technical reason, we assume that $X$ does not contain stacky points, so it consists of a finite number of smooth points. Then, after applying a coordinate change if necessary, $W$ can be written as

$$
W=x_{1} W_{1}+x_{2} W_{2}
$$

for some homogeneous elements $W_{i} \in A$ such that $x_{1}$ and $x_{2}$ do not divide $W_{2}$ and $W_{1}$, respectively. The heart $\mathcal{A}_{W}$ is described in the following way:

$$
\mathcal{A}_{W}=\left\langle\mathbb{C}\left(d-a_{1}-a_{2}-1\right), \cdots, \mathbb{C}(0), \Psi\left(\mathcal{O}_{x}\right): x \in X\right\rangle_{\mathrm{ex}} .
$$

Below we calculate the Hom groups between these generators. 


\section{GEPNER TYPE STABILITY CONDITIONS}

Lemma 3.15. For $0<j<d-a_{1}-a_{2}$, we have the following:

$$
\operatorname{Hom}_{\operatorname{HMF}^{i}(W)}^{i}(\mathbb{C}(j), \mathbb{C}(0)) \cong\left\{\begin{array}{cl}
R_{j}^{\vee}, & (i, j)=\left(1, a_{1}\right),\left(1, a_{2}\right) \\
R_{0}^{\vee}, & (i, j)=\left(2, a_{1}+a_{2}\right) \\
0, & \text { otherwise. }
\end{array}\right.
$$

Moreover, the natural map

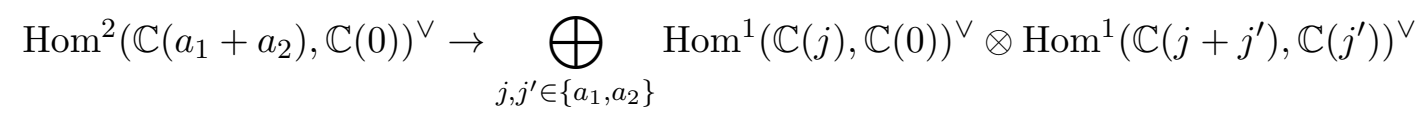

sends $1 \in R_{0}$ to $x_{1} \otimes x_{2}-x_{2} \otimes x_{1}$ under the isomorphism (34).

Proof. By the same argument as for Proposition 3.8, we have

$$
\operatorname{Hom}_{\mathrm{HMF}^{\mathrm{gr}}(W)}^{i}(\mathbb{C}(j), \mathbb{C}(0)) \cong \operatorname{Ext}_{\mathrm{gr}-R}^{i}(\mathbb{C}(j), \mathbb{C}(0)) .
$$

Then (34) is easily obtained by computing the right-hand side of (36) using the resolution

$$
\begin{aligned}
\cdots \rightarrow R\left(-a_{1}-d\right) \oplus R\left(-a_{2}-d\right) & \stackrel{h^{\prime}}{\rightarrow} R(-d) \oplus R\left(-a_{1}-a_{2}\right) \\
& \stackrel{h}{\rightarrow} R\left(-a_{1}\right) \oplus R\left(-a_{2}\right) \stackrel{\left(x_{1}, x_{2}\right)}{\rightarrow} R \rightarrow \mathbb{C}(0) \rightarrow 0 .
\end{aligned}
$$

Here $h$ and $h^{\prime}$ are given by matrices:

$$
h=\left(\begin{array}{cc}
W_{1} & -x_{2} \\
W_{2} & x_{1}
\end{array}\right), \quad h^{\prime}=\left(\begin{array}{cc}
x_{1} & x_{2} \\
-W_{2} & W_{1}
\end{array}\right) .
$$

Next, we consider the map (35). We write $W_{1}, W_{2}$ as

$$
W_{1}=x_{1} W_{11}+x_{2} W_{12}, \quad W_{2}=x_{1} W_{21}+x_{2} W_{21}
$$

for homogeneous elements $W_{k, l} \in A$. Let $x_{k}^{\vee} \in R_{a_{k}}^{\vee}$ be the dual basis of $x_{k} \in R_{a_{k}}$. We regard them as elements of the right-hand side of $(36)$ for $(i, j)=\left(1, a_{k}\right)$. Then $x_{1}^{\vee}$ is represented by the morphism of complexes

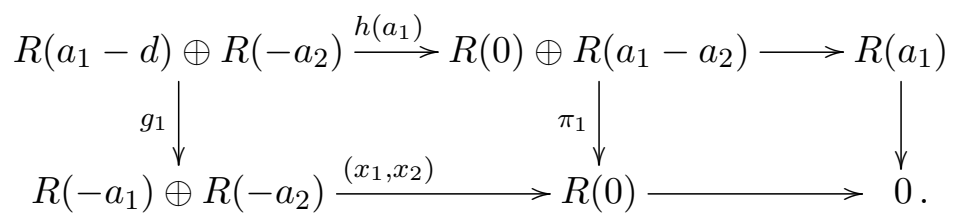

Similarly, $x_{2}^{\vee}$ is represented by

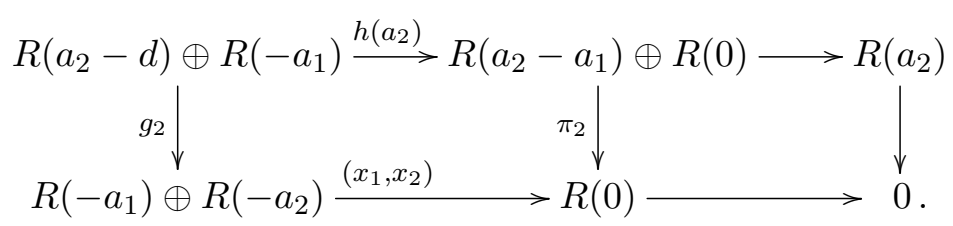

Here $\pi_{i}$ are projections onto the $i$ th factor, and the $g_{i}$ are given by matrices:

$$
g_{1}=\left(\begin{array}{cc}
W_{11} & 0 \\
W_{12} & -1
\end{array}\right), \quad g_{2}=\left(\begin{array}{ll}
W_{21} & 1 \\
W_{22} & 0
\end{array}\right) .
$$

The image of $x_{2}^{\vee} \otimes x_{1}^{\vee}$ by the dual of (35) is computed by composing these morphisms of complexes. By restricting the map $\pi_{2} \circ g_{1}\left(a_{2}\right)$ to the second component of $R\left(a_{1}+a_{2}-d\right) \oplus R(0)$, we see that $x_{2}^{\vee} \otimes x_{1}^{\vee}$ is mapped to $-1^{\vee}$, where $1^{\vee}$ is the dual basis of $1 \in R_{0}$. Similarly, $x_{1}^{\vee} \otimes x_{2}^{\vee}$ is mapped to $1^{\vee}$. By dualizing, we obtain the result. 


\section{YUKINOBU TODA}

Remark 3.16. By (34) and (36), an element $u \in R_{a_{i}}^{\vee}$ determines the extension

$$
0 \rightarrow \mathbb{C}(0) \rightarrow M_{u} \rightarrow \mathbb{C}\left(a_{i}\right) \rightarrow 0
$$

in gr- $R$. The graded $R$-module $M_{u}$ is isomorphic to $\mathbb{C}(0) \oplus \mathbb{C}\left(a_{i}\right)$ as a graded $\mathbb{C}$-vector space, and the action of $x_{i}$ is given by sending $1 \in \mathbb{C}(0)$ to $u\left(x_{i}\right) \in \mathbb{C}\left(a_{i}\right)$.

Next, we compute the Hom groups between $\mathbb{C}(j)$ and $\Psi\left(\mathcal{O}_{x}\right)$ for closed points $x \in X$. Let $M(x)$ be the graded $R$-module defined by (31). We have the following lemma.

Lemma 3.17. Suppose $0 \leqslant j<d-a_{1}-a_{2}$, and that $x \in X$ is represented by $\left(p_{1}, p_{2}\right) \in \mathbb{C}^{2}$. Then we have

$$
\operatorname{Hom}_{\mathrm{HMF}^{\mathrm{gr}}(W)}^{i}\left(\mathbb{C}(j), \Psi\left(\mathcal{O}_{x}\right)\right) \cong\left\{\begin{array}{cl}
\mathbb{C} u_{j}, & i=1, j \in\left[0, a_{2}\right) \\
\mathbb{C} v_{j}, & i=2, j \in\left[a_{1}, a_{1}+a_{2}\right) \\
0, & \text { otherwise. }
\end{array}\right.
$$

Here $u_{j}$ and $v_{j}$ are regarded as elements

$$
\begin{aligned}
& u_{j}=p_{1} e_{-j+a_{1}} \oplus p_{2} e_{-j+a_{2}} \in M(x)_{-j+a_{1}} \oplus M(x)_{-j+a_{2}} \\
& v_{j}=v e_{-j+d} \oplus e_{-j+a_{1}+a_{2}} \in M(x)_{-j+d} \oplus M(x)_{-j+a_{1}+a_{2}},
\end{aligned}
$$

where $v:=W_{2}\left(p_{1}, p_{2}\right) / p_{1}=-W_{1}\left(p_{1}, p_{2}\right) / p_{2}$. If $j \in\left[a_{1}, a_{1}+a_{2}\right)$, the natural map

$$
\operatorname{Hom}^{2}\left(\mathbb{C}(j), \Psi\left(\mathcal{O}_{x}\right)\right)^{\vee} \rightarrow \underset{j^{\prime} \in\left\{j-a_{1}, j-a_{2}\right\}}{\bigoplus} \operatorname{Hom}^{1}\left(\mathbb{C}(j), \mathbb{C}\left(j^{\prime}\right)\right)^{\vee} \otimes \operatorname{Hom}\left(\mathbb{C}\left(j^{\prime}\right), \Psi\left(\mathcal{O}_{x}\right)\right)^{\vee}
$$

sends the dual basis $v_{j}^{\vee}$ to $p_{2} x_{1} \otimes u_{j-a_{1}}^{\vee}-p_{1} x_{2} \otimes u_{j-a_{2}}^{\vee}$ under the isomorphisms (34), (39). (Here we set $u_{j-a_{1}}^{\vee}=0$ if $j-a_{1} \geqslant a_{1}$.)

Proof. Similarly to the proof of Lemma 3.15, we have the isomorphism

$$
\operatorname{Hom}_{\operatorname{HMF}^{g r}(W)}^{i}\left(\mathbb{C}(j), \Psi\left(\mathcal{O}_{x}\right)\right) \cong \operatorname{Ext}_{\text {gr- } R}^{i}(\mathbb{C}(j), M(x)) .
$$

Applying $\operatorname{Hom}_{\mathrm{gr}-R}(*, M(x)(-j))$ to the exact sequence (37), the right-hand side of (41) is computed by the $i$ th cohomology group of the following complex:

$$
\begin{aligned}
0 \rightarrow M(x)_{-j} & \stackrel{\left(p_{1}, p_{2}\right)}{\longrightarrow} M(x)_{-j+a_{1}} \oplus M(x)_{-j+a_{2}} \\
& \stackrel{{ }^{t} h\left(p_{1}, p_{2}\right)}{\longrightarrow} M(x)_{-j+d} \oplus M(x)_{-j+a_{1}+a_{2}} \\
& \stackrel{{ }^{t} h^{\prime}}{\stackrel{\left(p_{1}, p_{2}\right)}{\longrightarrow}} M(x)_{-j+d+a_{1}} \oplus M(x)_{-j+d+a_{2}} \rightarrow \cdots .
\end{aligned}
$$

Here $h\left(p_{1}, p_{2}\right), h^{\prime}\left(p_{1}, p_{2}\right)$ are the substitution of $\left(x_{1}, x_{2}\right)=\left(p_{1}, p_{2}\right)$ in the matrices (38). Then (39) easily follows by noting that all non-zero maps in the complex (42) are of rank one. The image of $v_{j}^{\vee}$ by the map (40) is computed similarly to (35), so we omit the details.

These computations enable us to describe $\mathcal{A}_{W}$ in terms of quiver representations with relations. Here we only discuss the case $a_{1}=a_{2}=1$. Recall that for a set of objects $\left(F_{N}, \cdots, F_{2}, F_{1}\right)$, the ext-quiver $\mathcal{Q}\left(F_{\bullet}\right)$ is defined as follows: the set of vertices is

$$
\{1,2, \cdots, N\}
$$

and the number of edges from $j$ to $j^{\prime}$ is the dimension of $\operatorname{Ext}^{1}\left(F_{j}, F_{j^{\prime}}\right)$, which we identify with a basis of $\operatorname{Ext}^{1}\left(F_{j}, F_{j^{\prime}}\right)^{\vee}$. The following lemma may be well known, but we include the proof later in Subsection 3.6, because of the lack of a reference. 


\section{GEPNER TYPE STABILITY CONDITIONS}

Lemma 3.18. Let $\mathcal{D}$ be a triangulated category with finite dimensional Hom spaces, generated by an ext-exceptional collection $\left(F_{N}, \cdots, F_{2}, F_{1}\right)$. Let $\mathcal{A}$ be the heart of a bounded t-structure on $\mathcal{D}$ given by the extension closure of all $F_{i}$ for $1 \leqslant i \leqslant N$. Suppose that there is a partition

$$
\{1, \cdots, N\}=P_{1} \sqcup \cdots \sqcup P_{l}, j^{\prime}>j \quad \text { if } \quad j \in P_{k}, j^{\prime} \in P_{k^{\prime}}, k^{\prime}>k .
$$

such that, by setting $\widehat{F}_{k}:=\oplus_{j \in P_{k}} F_{j}$, the following condition holds:

$$
\operatorname{Ext}^{i}\left(\widehat{F}_{k^{\prime}}, \widehat{F}_{k}\right)=0 \quad \text { unless }\left(i, k^{\prime}-k\right)=(1,1),(2,2) .
$$

Then $\mathcal{A}$ is equivalent to the abelian category of $\mathcal{Q}\left(F_{\bullet}\right)$-representations with relations generated by the images of the following natural maps for all $1 \leqslant k \leqslant l$ :

$$
\operatorname{Ext}^{2}\left(\widehat{F}_{k+2}, \widehat{F}_{k}\right)^{\vee} \rightarrow \operatorname{Ext}^{1}\left(\widehat{F}_{k+2}, \widehat{F}_{k+1}\right)^{\vee} \otimes \operatorname{Ext}^{1}\left(\widehat{F}_{k+1}, \widehat{F}_{k}\right)^{\vee}
$$

The following corollary directly follows from Lemma 3.15, Lemma 3.17 and Lemma 3.18.

Corollary 3.19. Suppose that $a_{1}=a_{2}=1$ and write

$$
X=\left\{p^{(i)}=\left(p_{1}^{(i)}, p_{2}^{(i)}\right) \in \mathbb{P}^{1}: 1 \leqslant i \leqslant d\right\} .
$$

Then $\mathcal{A}_{W}$ is equivalent to the category of representations of the quiver of the form

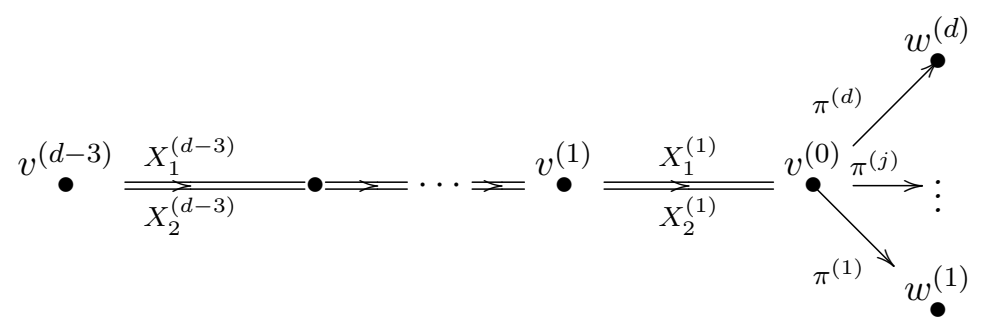

with relations given by

$$
X_{2}^{(i-1)} X_{1}^{(i)}=X_{1}^{(i-1)} X_{2}^{(i)}, \quad p_{2}^{(j)} \pi^{(j)} X_{1}^{(1)}=p_{1}^{(j)} \pi^{(j)} X_{2}^{(1)}
$$

for all $2 \leqslant i \leqslant d-3$ and $1 \leqslant j \leqslant d$. The vertex $v^{(i)}$ corresponds to $\mathbb{C}(i)$ and $w^{(j)}$ corresponds to $\Psi\left(\mathcal{O}_{p^{(j)}}\right)$.

By investigating the filtration (33), we are able to describe $\mathbb{C}(-\varepsilon)[-1]$ in terms of a representation of the quiver (45). The following corollary is a straightforward adaptation of Corollary 3.19, Remark 3.14 and Remark 3.16.

Corollary 3.20. In the situation of Corollary 3.19, the object $\mathbb{C}(-\varepsilon)[-1]$ in $\mathcal{A}_{W}$ is the representation of the quiver (45) given as follows:

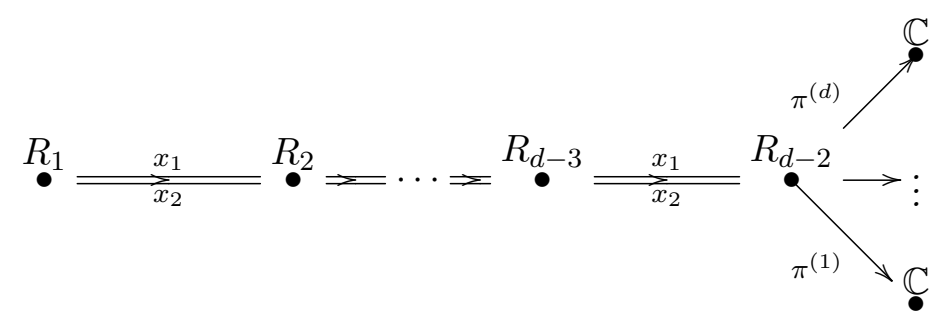

Here $\pi^{(j)}: R_{d-2} \rightarrow \mathbb{C}$ is the evaluation at $p^{(j)}=\left(p_{1}^{(j)}, p_{2}^{(j)}\right)$. 


\section{YuKINOBU TODA}

\subsection{Description of $\mathcal{A}_{W}$ via coherent systems}

In this subsection, we assume that $\varepsilon=-1$ and describe the heart $\mathcal{A}_{W}$ in terms of coherent systems on $X$. Let us recall the definition of coherent systems.

Definition 3.21. A coherent system on a Deligne-Mumford stack $X$ is data

$$
V \otimes \mathcal{O}_{X} \stackrel{s}{\rightarrow} F
$$

where $V$ is a finite dimensional $\mathbb{C}$-vector space, $F \in \operatorname{Coh}(X)$ and $s$ is a morphism in $\operatorname{Coh}(X)$.

The category of coherent systems on $X$ is denoted by $\operatorname{Syst}(X)$. The set of morphisms is given by the commutative diagrams

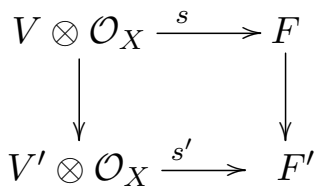

in $\operatorname{Coh}(X)$. Obviously, $\operatorname{Syst}(X)$ is an abelian category. We have the following proposition.

Proposition 3.22. Suppose that $\varepsilon=-1$. Then we have an equivalence of abelian categories

$$
\Theta: \operatorname{Syst}(X) \stackrel{\sim}{\rightarrow} \mathcal{A}_{W} .
$$

Proof. Let us take a coherent system $\left(V \otimes \mathcal{O}_{X} \stackrel{s}{\rightarrow} F\right)$ on $X$. By Lemma 3.12, the morphism $s$ is regarded as an element

$$
\bar{s} \in \operatorname{Hom}_{\mathrm{HMF}^{\mathrm{gr}}(W)}(V \otimes \mathbb{C}(0), \Psi(F)[1]) .
$$

The cone of $\bar{s}$ determines an object in $\mathcal{A}_{W}$, which fits into the exact sequence

$$
0 \rightarrow \Psi(F) \rightarrow \operatorname{Cone}(\bar{s}) \rightarrow V \otimes \mathbb{C}(0) \rightarrow 0
$$

in $\mathcal{A}_{W}$. The correspondence

$$
\Theta:(F, s) \mapsto \operatorname{Cone}(\bar{s})
$$

is a functor from $\operatorname{Syst}(X)$ to $\mathcal{A}_{W}$. Indeed, if we are given a commutative diagram (47), the induced morphism

$$
\operatorname{Cone}(\bar{s}) \rightarrow \operatorname{Cone}\left(\bar{s}^{\prime}\right)
$$

is uniquely determined by the vanishing $\operatorname{Hom}(\mathbb{C}(0), \Psi \operatorname{Coh}(X))=0$. This vanishing is proved using the same argument as for Proposition 3.8.

Conversely, let us take an object $E \in \mathcal{A}_{W}$. There is an exact sequence

$$
0 \rightarrow \Psi(F) \rightarrow E \rightarrow V \otimes \mathbb{C}(0) \rightarrow 0
$$

in $\mathcal{A}_{W}$ for a finite dimensional vector space $V$ and $F \in \operatorname{Coh}(X)$. By Lemma 3.12, the extension class $\xi$ of (48) is regarded as an element

$$
\xi^{\prime} \in \operatorname{Hom}\left(V \otimes \mathcal{O}_{X}, F\right) .
$$

The pair $\left(F, \xi^{\prime}\right)$ determines an object in $\operatorname{Syst}(X)$. The correspondence

$$
\Theta^{\prime}: E \mapsto\left(F, \xi^{\prime}\right)
$$

is a functor from $\mathcal{A}_{W}$ to $\operatorname{Syst}(X)$ since $\left\langle\mathbb{C}(0), \Psi D^{b} \operatorname{Coh}(X)\right\rangle$ is a semi-orthogonal decomposition of $\operatorname{HMF}^{\mathrm{gr}}(W)$.

Obviously, we have

$$
\Theta^{\prime} \circ \Theta \cong \operatorname{id}_{\operatorname{Syst}(X)}, \quad \Theta \circ \Theta^{\prime} \cong \operatorname{id}_{\mathcal{A}_{W}} ;
$$




\section{GEPNER TYPE STABILITY CONDITIONS}

hence $\Theta$ is an equivalence.

Combining this with Lemma 3.11 and Remark 3.14, we immediately obtain the following corollary.

Corollary 3.23. Suppose $\varepsilon=-1$. Then the object $\mathbb{C}(1)[-1] \in \mathcal{A}_{W}$ is given by

$$
\mathbb{C}(1)[-1] \cong \Theta\left(H^{0}\left(X, \mathcal{O}_{X}(1)\right) \otimes \mathcal{O}_{X} \stackrel{s}{\rightarrow} \mathcal{O}_{X}(1)\right) .
$$

Here $s$ is the canonical evaluation morphism.

\subsection{Proof of Lemma 3.18}

Finally, in this section, we give a proof of Lemma 3.18. The proof is straightforward, and probably well known. We recommend the readers to skip this subsection at the first reading.

Proof. We denote by $I$ the set of relations generated by the images of $(44)$. Let $\operatorname{Rep}\left(\mathcal{Q}\left(F_{\bullet}\right), I\right)$ be the category of $\mathcal{Q}\left(F_{\bullet}\right)$-representations with relation $I$. We divide the proof into three steps.

STEP 1.

We construct the functor $\Phi: \mathcal{A} \rightarrow \operatorname{Rep}\left(\mathcal{Q}\left(F_{\bullet}\right), I\right)$ in the following way: any object $E \in \mathcal{A}$ admits a filtration

$$
0=E_{0} \subset E_{1} \subset \cdots \subset E_{l}=E
$$

such that $E_{k} / E_{k-1}$ can be written as

$$
E_{k} / E_{k-1} \cong \bigoplus_{j \in P_{k}} F_{j} \otimes V_{j}
$$

for finite dimensional vector spaces $V_{j}$. By the exact sequence

$$
0 \rightarrow E_{k} / E_{k-1} \rightarrow E_{k+1} / E_{k-1} \rightarrow E_{k+1} / E_{k} \rightarrow 0
$$

we obtain linear maps

$$
\phi_{j^{\prime} j}: V_{j^{\prime}} \otimes \operatorname{Ext}^{1}\left(F_{j^{\prime}}, F_{j}\right)^{\vee} \rightarrow V_{j}
$$

for $j^{\prime} \in P_{k+1}, j \in P_{k}$, which defines the $\mathcal{Q}\left(F_{\bullet}\right)$-representation $\Phi(E)$. In order to show that the representation $\Phi(E)$ satisfies the relation $I$, consider the composition of extension classes of (49):

$$
\bigoplus_{j^{\prime \prime} \in P_{k+2}} F_{j^{\prime \prime}} \otimes V_{j^{\prime \prime}} \rightarrow \bigoplus_{j^{\prime} \in P_{k+1}} F_{j^{\prime}} \otimes V_{j^{\prime}}[1] \rightarrow \bigoplus_{j \in P_{k}} F_{j} \otimes V_{j}[2]
$$

This composition must vanish since it coincides with the composition

$$
E_{k+2} / E_{k+1} \rightarrow E_{k+1} / E_{k-1}[1] \rightarrow E_{k+1} / E_{k}[1] \rightarrow E_{k} / E_{k-1}[2]
$$

where the first morphism corresponds to the extension class of

$$
0 \rightarrow E_{k+1} / E_{k-1} \rightarrow E_{k+2} / E_{k-1} \rightarrow E_{k+2} / E_{k+1} \rightarrow 0 .
$$

By applying $\operatorname{Hom}\left(F_{j^{\prime \prime}}, *\right)$ for $j^{\prime \prime} \in P_{k+2}$ to $(51)$, taking the adjunction and the $\left(j^{\prime \prime}, j\right)$-component for $j \in P_{k}$, we see that the map

$$
V_{j^{\prime \prime}} \otimes \bigoplus_{j^{\prime} \in P_{k+1}}\left(\operatorname{Ext}^{1}\left(F_{j^{\prime \prime}}, F_{j^{\prime}}\right)^{\vee} \otimes \operatorname{Ext}^{1}\left(F_{j^{\prime}}, F_{j}\right)^{\vee}\right) \rightarrow V_{j}
$$




\section{YukinOBU TODA}

given by the sum of compositions

$$
\sum_{j^{\prime} \in P_{k+1}} \phi_{j^{\prime} j} \circ \phi_{j^{\prime \prime} j^{\prime}}
$$

is zero on $V_{j^{\prime \prime}} \otimes I_{j^{\prime \prime}, j}$, where $I_{j^{\prime \prime}, j}$ is the image of (44) restricted to the $\operatorname{Ext}^{2}\left(F_{j^{\prime \prime}} \cdot F_{j}\right)^{\vee}$-component. This implies that $\Phi(E)$ satisfies the relation $I$, hence it is an object in $\operatorname{Rep}\left(\mathcal{Q}\left(F_{\bullet}\right), I\right)$.

\section{STEP 2.}

The correspondence $E \mapsto \Phi(E)$ obviously determines a fully faithful functor from $\mathcal{A}$ to $\operatorname{Rep}\left(\mathcal{Q}\left(F_{\bullet}\right), I\right)$, since $\left(F_{N}, \cdots, F_{2}, F_{1}\right)$ is an ext-exceptional collection. It remains to show that $\Phi$ is essentially surjective. Let us take an object

$$
W \in \operatorname{Rep}\left(\mathcal{Q}\left(F_{\bullet}\right), I\right) .
$$

It consists of finite dimensional vector spaces $V_{j}$ for $1 \leqslant j \leqslant N$ and linear maps (50) whose composition (52) is zero on $V_{j^{\prime \prime}} \otimes I_{j^{\prime \prime}, j}$ for $\left(j^{\prime \prime}, j\right) \in P_{k+2} \times P_{k}$. We need to show the existence of and $E \in \mathcal{A}$ such that $\Phi(E) \cong W$.

By induction on $l$, we may assume that the assertion holds for $l-1$. We set full subcategories $\mathcal{A}_{k} \subset \mathcal{D}$ as follows:

$$
\mathcal{A}_{k}:=\left\langle F_{j}: j \in P_{k^{\prime}}, 1 \leqslant k^{\prime} \leqslant k\right\rangle_{\mathrm{ex}} .
$$

Let $\mathcal{Q}\left(F_{\bullet}^{\prime}\right)$ be the ext-quiver for $\mathcal{A}_{l-1}$ and define the relation $I^{\prime}$ by restricting $I$ to $\mathcal{Q}\left(F_{\bullet}^{\prime}\right)$. The category $\operatorname{Rep}\left(\mathcal{Q}\left(F_{\bullet}^{\prime}\right), I^{\prime}\right)$ can be naturally considered as a subcategory of $\operatorname{Rep}\left(\mathcal{Q}\left(F_{\bullet}\right), I\right)$, and there is an exact sequence

$$
0 \rightarrow W^{\prime} \rightarrow W \rightarrow W_{l} \rightarrow 0,
$$

where $W^{\prime} \in \operatorname{Rep}\left(\mathcal{Q}\left(F_{\bullet}^{\prime}\right), I^{\prime}\right)$ and $W_{l}$ can be written as

$$
W_{l} \cong \bigoplus_{j \in P_{l}} e_{j} \otimes V_{j}
$$

Here $e_{j}$ is the simple object in $\operatorname{Rep}\left(\mathcal{Q}\left(F_{\bullet}\right), I\right)$ corresponding to the vertex $j$.

By the induction hypothesis, there is an object $E^{\prime} \in \mathcal{A}_{l-1}$ such that $\Phi\left(E^{\prime}\right) \cong W^{\prime}$. By the exact sequence (53), it is enough to show that the map

$$
\alpha: \operatorname{Ext}_{\mathcal{A}}^{1}\left(F_{j}, E^{\prime}\right) \rightarrow \operatorname{Ext}_{\text {Rep }}^{1}\left(e_{j}, W^{\prime}\right)
$$

induced by $\Phi$ is an isomorphism for all $j \in P_{l}$. Here we have written $\operatorname{Rep}\left(\mathcal{Q}\left(F_{\bullet}\right), I\right)$ as $\operatorname{Rep}$ for the sake of simplicity.

\section{STEP 3.}

We show that the morphism (54) is an isomorphism. Let us consider the exact sequence

$$
0 \rightarrow E^{\prime \prime} \rightarrow E^{\prime} \rightarrow E_{l-1} \rightarrow 0
$$

in $\mathcal{A}$ with $E^{\prime \prime} \in \mathcal{A}_{l-2}$ and where $E_{l-1}$ is given by

$$
E_{l-1} \cong \bigoplus_{j^{\prime} \in P_{l-1}} F_{j^{\prime}} \otimes V_{j^{\prime}}
$$

By condition (43), for $j \in P_{l}$, we see that $\operatorname{Ext}_{\mathcal{A}}^{1}\left(F_{j}, E^{\prime \prime}\right)=0$ and there is a natural isomorphism

$$
\operatorname{Ext}_{\mathcal{D}}^{2}\left(F_{j}, E^{\prime \prime}\right) \cong \bigoplus_{j^{\prime \prime} \in P_{l-2}} \operatorname{Ext}_{\mathcal{D}}^{2}\left(F_{j}, F_{j^{\prime \prime}}\right) \otimes V_{j^{\prime \prime}} .
$$




\section{GePner type STABILITy CONDitions}

Therefore applying $\operatorname{Hom}\left(F_{j}, *\right)$ to $(55)$, we obtain the exact sequence

$$
\begin{aligned}
0 \rightarrow \operatorname{Ext}_{\mathcal{A}}^{1}\left(F_{j}, E^{\prime}\right) \rightarrow \bigoplus_{j^{\prime} \in P_{l-1}} & \operatorname{Ext}_{\mathcal{A}}^{1}\left(F_{j}, F_{j^{\prime}}\right) \otimes V_{j^{\prime}} \\
& \stackrel{\beta}{\rightarrow} \bigoplus_{j^{\prime \prime} \in P_{l-2}} \operatorname{Ext}_{\mathcal{D}}^{2}\left(F_{j}, F_{j^{\prime \prime}}\right) \otimes V_{j^{\prime \prime}} .
\end{aligned}
$$

On the other hand, there is an exact sequence in $\operatorname{Rep}\left(\mathcal{Q}\left(F_{\bullet}\right), I\right)$

$$
0 \rightarrow W^{\prime \prime} \rightarrow W^{\prime} \rightarrow \bigoplus_{j^{\prime} \in P_{l-1}} e_{j^{\prime}} \otimes V_{j^{\prime}} \rightarrow 0
$$

such that $W^{\prime \prime} \cong \Phi\left(E^{\prime \prime}\right)$. Since $\operatorname{Ext}_{\operatorname{Rep}}^{1}\left(e_{a}, e_{a^{\prime}}\right)=0$ unless $a \in P_{k}, a^{\prime} \in P_{k^{\prime}}$ with $k-k^{\prime}=1$, we have $\operatorname{Ext}_{\operatorname{Rep}}^{1}\left(e_{j}, W^{\prime \prime}\right)=0$ for any $j \in P_{l}$. Therefore we obtain the commutative diagram

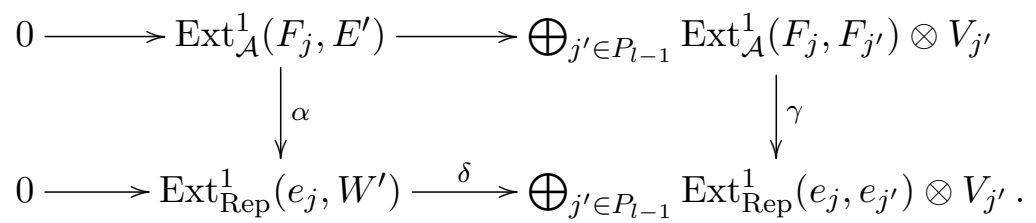

Here $\gamma$ is an isomorphism induced by $\Phi$. By this diagram, it follows that $\alpha$ is injective. On the other hand, the composition

$$
\beta \circ \gamma^{-1} \circ \delta: \operatorname{Ext}_{\mathrm{Rep}}^{1}\left(e_{j}, W^{\prime}\right) \rightarrow \bigoplus_{j^{\prime \prime} \in P_{l-2}} \operatorname{Ext}_{\mathcal{D}}^{2}\left(F_{j}, F_{j^{\prime \prime}}\right) \otimes V_{j^{\prime \prime}}
$$

vanishes, since any object given by an extension class in the left-hand side satisfies the relation $I$. Therefore $\alpha$ is surjective, hence is an isomorphism.

\section{Construction of Gepner type stability conditions}

In this section, we propose a general recipe for the construction of a desired Gepner type stability condition. We first compute the central charge $Z_{G}$ in terms of generators of $\mathcal{A}_{W}$, and try to construct $\sigma_{G}$ via a tilting of $\mathcal{A}_{W}$. In what follows we assume that the stack $X$ in (22) is a smooth projective variety, for example $X$ does not contain stacky points. As in the previous section, we denote by $\Psi:=\Psi_{1}$ the fully faithful functor from $D^{b} \operatorname{Coh}(X)$ to $\operatorname{HMF}^{\mathrm{gr}}(W)$ constructed by Orlov, which is an equivalence if $\varepsilon=0$.

\subsection{Computation of the central charge $(\varepsilon=0$ case)}

In this subsection, we explain how to compute $Z_{G}$ in the case $\varepsilon=0$. If $\varepsilon=0, X$ is a Calabi-Yau manifold of dimension $n-2$, and $\mathcal{A}_{W}$ is equivalent to $\operatorname{Coh}(X)$ via $\Psi$. Let us consider the group homomorphism given by

$$
Z_{G} \circ \Psi: K(X) \rightarrow \mathbb{C} .
$$

This group homomorphism can be described in terms of Chern characters on $K(X)$. Indeed, a fundamental theory on Hochschild homology groups implies that $\Psi$ induces an isomorphism 


\section{YuKINOBU TODA}

$\Psi_{*}: \mathrm{HH}_{0}(X) \cong \mathrm{HH}_{0}(W)$ such that the following diagram commutes (cf. [PV12, Section 1]):

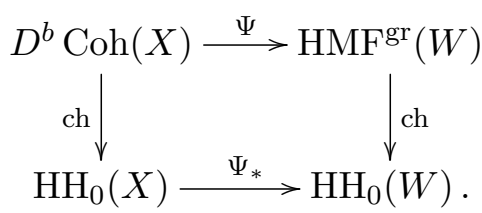

We also have the Hochschild-Kostant-Rosenberg isomorphism

$$
\mathrm{HH}_{0}(X) \stackrel{\cong}{\rightrightarrows} \mathrm{H} \Omega_{0}(X):=\bigoplus_{j=0}^{n-2} H^{j}\left(X, \Omega_{X}^{j}\right)
$$

whose composition with ch: $D^{b} \mathrm{Coh}(X) \rightarrow \mathrm{HH}_{0}(X)$ coincides with the classical Chern character map [Căl05, Theorem 4.5]. Since our central charge $Z_{G}$ factors through the Chern character map on $\mathrm{HMF}^{\mathrm{gr}}(W)$ (cf. Remark 2.16) and the Poincaré pairing on $\mathrm{H} \Omega_{0}(X)$ is perfect, the group homomorphism (57) can be written as

$$
E \mapsto \sum_{j=0}^{n-2} \int_{X} \alpha_{j} \cdot \operatorname{ch}_{j}(E)
$$

for some $\alpha_{j} \in H^{n-2-j, n-2-j}(X)$. Here by an abuse of notation, we also denote by $\operatorname{ch}(E) \in$ $\mathrm{H} \Omega_{0}(X)$ the classical Chern character of $E \in K(X)$, and by $\operatorname{ch}_{j}(E)$ its $H^{j, j}(X)$-component.

On the other hand, let us consider the autoequivalence $F$ of $D^{b} \operatorname{Coh}(X)$ defined by

$$
F:=\mathrm{ST}_{\mathcal{O}_{X}} \circ \otimes \mathcal{O}_{X}(1)
$$

By Proposition 3.2, the above autoequivalence corresponds to the grade shift functor $\tau$ on $\operatorname{HMF}^{g r}(W)$ via the equivalence $\Psi$. By the Riemann-Roch theorem, the autoequivalence $F$ acts on $\operatorname{ch}(E)$ for $E \in D^{b} \operatorname{Coh}(X)$ in the following way

$$
F_{*}: \operatorname{ch}(E) \mapsto e^{H} \operatorname{ch}(E)-\left(\int_{X} e^{H} \operatorname{ch}(E) \operatorname{td} X\right) \cdot 1 .
$$

Here $H$ is the first Chern class of $\mathcal{O}_{X}(1)$. This action naturally extends to a linear isomorphism on $\mathrm{H} \Omega_{0}(X)$, given by the composition of the matrices

$$
M:=\left(\begin{array}{cccc}
1-t_{n-2} & -t_{n-3} & \cdots & -t_{0} \\
0 & 1 & \cdots & 0 \\
\vdots & \vdots & \ddots & 0 \\
0 & 0 & \cdots & 1
\end{array}\right)\left(\begin{array}{cccc}
1 & 0 & \cdots & 0 \\
H & 1 & \cdots & 0 \\
\vdots & \vdots & \ddots & 0 \\
\frac{H^{n-2}}{(n-2) !} & \frac{H^{n-3}}{(n-3) !} & \cdots & 1
\end{array}\right) .
$$

Here $t_{j}$ is the $H^{j, j}(X)$-component of $\operatorname{td}_{X}$, and we regard an element in $\mathrm{H}_{0}(X)$ as a column vector. The Gepner type property of the central charge $Z_{G}$ can be translated into the following linear equation on $\alpha_{i}$ :

$$
\left(\alpha_{0}, \cdots, \alpha_{n-2}\right) \cdot M=e^{2 \pi \sqrt{-1} / d} \cdot\left(\alpha_{0}, \cdots, \alpha_{n-2}\right) .
$$

By Lemma 2.15, the solution space of (58) must be one dimensional, so it determines the group homomorphism (57) uniquely up to scalar multiplication.

In practice, it is more convenient to work with a smaller subspace in $\mathrm{H} \Omega_{0}(X)$. Let $\mathbb{C}\langle H\rangle$ be 


\section{GePner type STABILITy CONDitions}

the subspace in $\mathrm{H} \Omega_{0}(X)$ defined by

$$
\mathbb{C}\langle H\rangle:=\bigoplus_{j=0}^{n-2} \mathbb{C} H^{j}
$$

We have the following lemma.

Lemma 4.1. The solution space of (58) is contained in $\mathbb{C}\langle H\rangle$.

Proof. Let $\mathbb{C}\langle H\rangle^{\perp}$ be the orthogonal complement of $\mathbb{C}\langle H\rangle$ in $H \Omega_{0}(X)$ with respect to the Poincaré paring. We have the direct sum decomposition

$$
\mathrm{H} \Omega_{0}(X)=\mathbb{C}\langle H\rangle \oplus \mathbb{C}\langle H\rangle^{\perp}
$$

and $F_{*}$ preserves the direct summands. Since $\operatorname{td}_{X} \in \mathbb{C}\langle H\rangle, F_{*}$ acts on $\mathbb{C}\langle H\rangle^{\perp}$ via multiplication by $e^{H}$, which is unipotent. Hence all the eigenvectors of the action $F_{*}$ on $\mathbb{C}\langle H\rangle^{\perp}$ have eigenvalue 1 , which implies that the solution space of (58) is contained in $\mathbb{C}\langle H\rangle$.

By this lemma, it is enough to solve equation (58) for $\alpha_{j} \in \mathbb{C} H^{n-2-j}$. The ambiguity of the scalar multiplication is fixed by the following lemma.

Lemma 4.2. We have the equality

$$
\int_{X} \alpha_{0}=\prod_{j=1}^{n}\left(1-e^{-2 a_{j} \pi \sqrt{-1} / d}\right) .
$$

Proof. By Lemma 3.12, the object $\Psi^{L}(\mathbb{C}(0))$ is isomorphic to $\mathcal{O}_{X}[1]$. Since $\varepsilon=0$, the functor $\Psi$ is an equivalence, hence $\Psi^{L}=\Psi^{-1}$. It follows that $\Psi\left(\mathcal{O}_{X}\right)$ is isomorphic to $\mathbb{C}(0)[-1]$. Then equality (59) follows by applying homomorphism (57) to $\mathcal{O}_{X}$ and using the computation in Example 2.8.

Now, the $\alpha_{j} \in \mathbb{C} H^{n-2-j}$ are uniquely determined by equation (58) and the normalization (59). However, for our purpose, it is more convenient to consider a different normalization of $Z_{G} \circ \Psi$. Namely we write $Z_{G} \circ \Psi$ as a multiple of some non-zero complex number and a central charge on $\operatorname{HMF}^{\mathrm{gr}}(W)$ whose image of $\Psi\left(\mathcal{O}_{x}\right)$ is -1 . This is possible by the following lemma.

Lemma 4.3. For any $x \in X$, we have $Z_{G}\left(\Psi\left(\mathcal{O}_{x}\right)\right)=-C_{W}$, where $C_{W}$ is given by

$$
C_{W}:=-\left(1-e^{2 \pi \sqrt{-1} / d}\right)^{-1} \prod_{j=1}^{n}\left(1-e^{-2 a_{j} \pi \sqrt{-1} / d}\right)
$$

which satisfies

$$
C_{W} \in \mathbb{R}_{>0} e^{\sqrt{-1} \pi \theta_{W}}, \quad \theta_{W}=\frac{1}{2}(n-1)-\frac{1}{d}\left(\sum_{j=1}^{n} a_{j}+1\right) .
$$

Proof. Equality (60) follows from Example 2.8 and Lemma 3.10. Property (61) follows from

$$
1-e^{-2 \pi \sqrt{-1} \theta}=2 \sin \pi \theta \cdot e^{\left(\frac{1}{2}-\theta\right) \pi \sqrt{-1}} .
$$

We summarize the results in this subsection as follows. 


\section{YuKINOBU TODA}

Proposition 4.4. Suppose $\varepsilon=0$. Then for $E \in D^{b} \operatorname{Coh}(X)$, the central charge $Z_{G}(\Psi(E))$ can be written as

$$
Z_{G}(\Psi(E))=C_{W} \sum_{j=0}^{n-2} \int_{X} \alpha_{j}^{\dagger} \cdot \operatorname{ch}_{j}(E),
$$

where $\left(\alpha_{0}^{\dagger}, \cdots, \alpha_{n-2}^{\dagger}\right)$ satisfies $\alpha_{j}^{\dagger} \in \mathbb{C} H^{n-2-j}$; it is the unique solution of the linear equation

$$
\left(\alpha_{0}^{\dagger}, \cdots, \alpha_{n-2}^{\dagger}\right) \cdot M=e^{2 \pi \sqrt{-1} / d} \cdot\left(\alpha_{0}^{\dagger}, \cdots, \alpha_{n-2}^{\dagger}\right), \quad \alpha_{n-2}^{\dagger}=-1 .
$$

\subsection{Computation of the central charge $(\varepsilon<0$ case)}

The purpose of this subsection is to reduce the computation of $Z_{G}$ in the case $\varepsilon<0$ to that of the case $\varepsilon=0$. The strategy is to embed $X$ into a $n-2-\varepsilon$-dimensional Calabi-Yau manifold $\widehat{X}$ and relate $Z_{G}$ to the central charge on $\widehat{X}$. We set

$$
\widehat{A}:=\mathbb{C}\left[x_{1}, \cdots, x_{n}, x_{n+1}, \cdots, x_{n-\varepsilon}\right]
$$

and consider the element $\widehat{W} \in \widehat{A}$ defined by

$$
\widehat{W}:=W+x_{n+1}^{d}+\cdots+x_{n-\varepsilon}^{d} .
$$

Since we assume that $X$ does not contain stacky points, the stack

$$
\widehat{X}:=(\widehat{W}=0) \subset \mathbb{P}\left(a_{1}, \cdots, a_{n}, 1, \cdots, 1\right)
$$

also does not contain stacky points. The variety $\widehat{X}$ is a projective Calabi-Yau manifold of dimension $n-2-\varepsilon$ which contains $X$ as a zero locus $x_{n+1}=\cdots=x_{n-\varepsilon}=0$.

Let $\widehat{R}$ be the graded ring $\widehat{A} /(\widehat{W})$. There is a natural push-forward functor

$$
i_{*}: D_{\mathrm{sg}}^{\mathrm{gr}}(R) \rightarrow D_{\mathrm{sg}}^{\mathrm{gr}}(\widehat{R})
$$

by regarding a graded $R$-module as a graded $\widehat{R}$-module via the surjection $\widehat{R} \rightarrow R$ (cf. [Ued12]). Combining this with the equivalence (11) and the functor $\Psi=\Psi_{1}$ in (24), we obtain the diagram

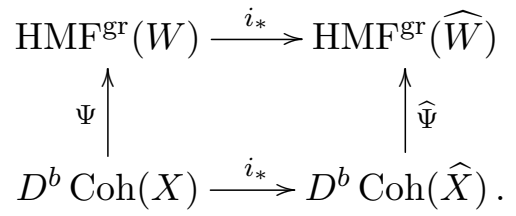

Here $i: X \rightarrow \widehat{X}$ is the inclusion and $\widehat{\Psi}$ is the equivalence obtained by applying the same construction as that of $\Psi$ to $\widehat{W}$ and $\widehat{X}$. We have the following lemma.

Lemma 4.5. The diagram (64) is commutative.

Proof. The result follows from the definitions of $\Psi, \widehat{\Psi}$ and the adjunction for $E \in D^{b} \operatorname{Coh}(X)$ :

$$
\bigoplus_{j \geqslant 1} \mathbf{R} \operatorname{Hom}_{\widehat{X}}\left(\mathcal{O}_{\widehat{X}}(j), i_{*} E\right) \cong \bigoplus_{j \geqslant 1} \mathbf{R} \operatorname{Hom}_{X}\left(\mathcal{O}_{X}(j), E\right)
$$

The top arrow $i_{*}$ of diagram (64) obviously commutes with grade shift functors on both sides. Also, by the functoriality of the Hochschild homologies, we have the push-forward functor

$$
i_{*}: \mathrm{HH}_{*}(W) \rightarrow \mathrm{HH}_{*}(\widehat{W})
$$




\section{GEPNER TYPE STABILITY CONDITIONS}

which preserves the one-dimensional eigenspaces in Lemma 2.15 on both sides. By Remark 2.16, the composition

$$
\widehat{Z}_{G} \circ i_{*}: K\left(\operatorname{HMF}^{\mathrm{gr}}(W)\right) \rightarrow K\left(\operatorname{HMF}^{\mathrm{gr}}(\widehat{W})\right) \rightarrow \mathbb{C}
$$

differs from $Z_{G}$ by a scalar constant, where $\widehat{Z}_{G}$ is the central charge (14) on $\operatorname{HMF}^{\mathrm{gr}}(\widehat{W})$ applied to $\widehat{W}$. Since $i_{*} \mathbb{C}(0)=\mathbb{C}(0)$, it follows that

$$
Z_{G}(P)=\left(1-e^{2 \pi \sqrt{-1} / d}\right)^{\varepsilon} \widehat{Z}_{G}\left(i_{*} P\right)
$$

for any $P \in \operatorname{HMF}^{\mathrm{gr}}(W)$, by comparing $Z_{G}(\mathbb{C}(0))$ and $\widehat{Z}_{G}(\mathbb{C}(0))$ as given in Example 2.8. In particular, using diagram (64), it follows that

$$
\begin{aligned}
Z_{G}\left(\Psi\left(\mathcal{O}_{x}\right)\right) & =\left(1-e^{2 \pi \sqrt{-1} / d}\right)^{-1} \prod_{j=1}^{n}\left(1-e^{2 a_{j} \pi \sqrt{-1} / d}\right) \\
& =:-C_{W},
\end{aligned}
$$

where $C_{W}$ coincides with the one defined in (60). As a summary, we have the following.

Proposition 4.6. Suppose $\varepsilon<0$. For $E \in D^{b} \operatorname{Coh}(X)$, the central charge $Z_{G}(\Psi(E))$ can be written as

$$
Z_{G}(\Psi(E))=C_{W} \sum_{j=0}^{n-2-\varepsilon} \int_{\widehat{X}} \widehat{\alpha}_{j}^{\dagger} \cdot \operatorname{ch}_{j}\left(i_{*} E\right),
$$

where $\left(\widehat{\alpha}_{0}^{\dagger}, \cdots, \widehat{\alpha}_{n-2-\varepsilon}^{\dagger}\right)$ satisfies

$$
\widehat{\alpha}_{j}^{\dagger} \in \mathbb{C} \widehat{H}^{n-2-\varepsilon-j}, \quad \widehat{H}:=c_{1}\left(\mathcal{O}_{\widehat{X}}(1)\right) ;
$$

it is the unique solution of equation (63) for $\widehat{X}$.

Note that $\widehat{\alpha}_{j}^{\dagger}$ is computed using the argument in the previous subsection, since $\widehat{X}$ is CalabiYau. Later, we will use the following data:

$$
\begin{aligned}
Z_{G}\left(\Psi\left(\mathcal{O}_{x}\right)\right) & =-C_{W} \\
& \in \mathbb{R}_{>0} e^{\sqrt{-1} \pi\left(\theta_{W}+1\right)} \\
Z_{G}(\mathbb{C}(j)) & =C_{W} e^{2 \pi j \sqrt{-1} / d}\left(1-e^{2 \pi \sqrt{-1} / d}\right) \\
& \in \mathbb{R}_{>0} e^{\sqrt{-1} \pi\left(\theta_{W}+\frac{1}{d}+\frac{2 j}{d}+\frac{3}{2}\right)} .
\end{aligned}
$$

Here $x \in X$ and $\theta_{W} \in \mathbb{Q}$ is defined by (61). The relation (66) is a consequence of the arguments above and the computation in Example 2.8.

\subsection{A recipe for constructing Gepner type stability conditions}

In this subsection, we explain how desired Gepner type stability conditions are constructed. We divide the construction into 3 steps: construction of a slope stability on $\mathcal{A}_{W}$, construction of $\sigma_{G}$ via a tilting of $\mathcal{A}_{W}$, and checking the Gepner type property of $\sigma_{G}$. In the next section, we will apply this recipe in the case $n-4 \leqslant \varepsilon \leqslant 0$.

STEP 1.

Our first step is to construct an analogue of a slope stability on $\mathcal{A}_{W}$. This is a map

$$
\mu: \mathcal{A}_{W} \rightarrow \mathbb{R} \cup\{ \pm \infty\}
$$




\section{YuKINOBU TODA}

satisfying the weak seesaw property: for any exact sequence $0 \rightarrow F \rightarrow E \rightarrow G \rightarrow 0$ in $\mathcal{A}_{W}$, we have either

$$
\begin{aligned}
& \mu(F) \leqslant \mu(E) \leqslant \mu(G) \text { or } \\
& \mu(F) \geqslant \mu(E) \geqslant \mu(G) .
\end{aligned}
$$

This slope function defines the $\mu$-stability in $\mathcal{A}_{W}$.

Definition 4.7. An object $E \in \mathcal{A}_{W}$ is $\mu$-stable or $\mu$-semi-stable if for any exact sequence $0 \rightarrow F \rightarrow E \rightarrow G \rightarrow 0$ in $\mathcal{A}_{W}$, we have the inequality

$$
\mu(F)<\mu(G) \text { or } \quad \mu(F) \leqslant \mu(G),
$$

respectively.

We require that $\mu$-stability satisfy the Harder-Narasimhan property; that is, for any $E \in \mathcal{A}_{W}$, there is a filtration

$$
0=E_{0} \subset E_{1} \subset \cdots \subset E_{N}=E
$$

in $\mathcal{A}_{W}$ such that each subquotient $F_{i}=E_{i} / E_{i-1}$ is $\mu$-semi-stable with $\mu\left(F_{i}\right)>\mu\left(F_{i+1}\right)$ for all $i$.

STEP 2.

Suppose that there is a slope function $\mu$ as above. We define a pair of full subcategories $\left(\mathcal{T}_{\mu}, \mathcal{F}_{\mu}\right)$ by setting

$$
\begin{aligned}
& \mathcal{T}_{\mu}:=\left\langle E \in \mathcal{A}_{W}: E \text { is } \mu \text {-semi-stable with } \mu(E)>0\right\rangle_{\mathrm{ex}} \\
& \mathcal{F}_{\mu}:=\left\langle E \in \mathcal{A}_{W}: E \text { is } \mu \text {-semi-stable with } \mu(E) \leqslant 0\right\rangle_{\mathrm{ex}} .
\end{aligned}
$$

The existence of Harder-Narasimhan filtrations in $\mu$-stability implies that $\left(\mathcal{T}_{\mu}, \mathcal{F}_{\mu}\right)$ is a torsion pair on $\mathcal{A}_{W}$ (cf. [HRS96]). We define $\mathcal{A}_{G}$ to be the associated tilting:

$$
\mathcal{A}_{G}:=\left\langle\mathcal{F}_{\mu}, \mathcal{T}_{\mu}[-1]\right\rangle_{\mathrm{ex}} \subset \operatorname{HMF}^{\mathrm{gr}}(W) .
$$

The category $\mathcal{A}_{G}$ is the heart of a bounded t-structure on $\operatorname{HMF}^{\mathrm{gr}}(W)$. We try to construct a desired stability condition from the heart $\mathcal{A}_{G}$, as follows.

Definition 4.8. We say that a triple

$$
\left(Z_{G}, \mathcal{A}_{G}, \theta\right), \quad \theta \in \mathbb{R}
$$

determines a stability condition if the following conditions hold:

- For any $0 \neq E \in \mathcal{A}_{G}$, we have

$$
Z_{G}(E) \in\left\{r e^{\sqrt{-1} \pi \phi}: r>0, \phi \in(\theta, \theta+1]\right\} .
$$

- Any object in $\mathcal{A}_{W}$ admits a Harder-Narasimhan filtration with respect to $Z_{G}$-stability.

Here $Z_{G}$-stability and its Harder-Narasimhan filtrations are defined in the same way as in the $\mu$-stability, by replacing $\mu$ by $\arg Z_{G}(*) \in(\theta, \theta+1$ ]. If the triple (70) determines a stability condition, we associate with it a pair

$$
\sigma_{G}=\left(Z_{G},\left\{\mathcal{P}_{G}(\phi)\right\}_{\phi \in \mathbb{R}}\right), \quad \mathcal{P}_{G}(\phi) \subset \operatorname{HMF}^{\mathrm{gr}}(W)
$$

in the following way: we define $\mathcal{P}_{G}(\phi)$ for $\phi \in(\theta, \theta+1]$ to be

$$
\mathcal{P}_{G}(\phi)=\left\{E \in \mathcal{A}_{W}: Z_{G} \text {-semi-stable with } Z_{G}(E) \in \mathbb{R}_{>0} e^{\sqrt{-1} \pi \phi}\right\} \cup\{0\}
$$




\section{Gepner type Stability CONDitions}

and other $\mathcal{P}_{G}(\phi)$ are determined by the rule

$$
\mathcal{P}_{G}(\phi+1)=\mathcal{P}_{G}(\phi)[1] .
$$

If $\theta=0$, this construction is nothing but the one given in [Bri07, Proposition 5.3], and the same argument applies to show that (72) is a stability condition. Below, for an interval $I \subset \mathbb{R}$, we set

$$
\mathcal{P}_{G}(I):=\left\langle\mathcal{P}_{G}(\phi): \phi \in I\right\rangle_{\mathrm{ex}} .
$$

Note that $\mathcal{P}_{G}((\theta, \theta+1])$ coincides with $\mathcal{A}_{G}$ by our construction. We require the local finiteness of our stability condition; that is, for any $\phi \in \mathbb{R}$, the quasi-abelian category $\mathcal{P}_{G}((\phi-\delta, \phi+\delta))$ is noetherian and artinian for $0<\delta \ll 1$ (cf. [Bri07, Definition 5.7]). It in particular implies that any object $E \in \mathcal{P}_{G}(\phi)$ admits a Jordan-Hölder filtration.

Remark 4.9. The local finiteness condition holds if the image of $Z_{G}$ is discrete. By Remark 2.10, this is always satisfied in the cases studied in the next section, so we do not need to consider the local finiteness.

\section{STEP 3.}

In this step, we assume that the triple (70) determines a stability condition $\sigma_{G}$. We expect that $\sigma_{G}$ is a Gepner type stability condition with respect to $(\tau, 2 / d)$. To show this, we consider the following stability condition:

$$
\tau_{*}^{-1} \sigma_{G}\left(\frac{2}{d}\right)=\left(Z_{G},\left\{\mathcal{P}_{G}^{\prime}(\phi)\right\}_{\phi \in \mathbb{R}}\right),
$$

where $\mathcal{P}_{G}^{\prime}(\phi)$ is given by

$$
\mathcal{P}_{G}^{\prime}(\phi)=\tau^{-1} \mathcal{P}_{G}\left(\phi+\frac{2}{d}\right) .
$$

It is enough to show that $(73)$ coincides with $\sigma_{G}$. This is equivalent to having $\mathcal{P}_{G}^{\prime}((\theta, \theta+1])=$ $\mathcal{P}_{G}((\theta, \theta+1])$, or, equivalently,

$$
\tau\left(\mathcal{A}_{G}\right)=\mathcal{P}_{G}\left(\left(\theta+\frac{2}{d}, \theta+\frac{2}{d}+1\right]\right)
$$

We show equality (74) by investigating the $\sigma_{G}$-stability of simple objects in $\mathcal{A}_{W}$. When $n=2$, we have the following lemma.

Lemma 4.10. Suppose $n=2$ and that the following inequality holds:

$$
\theta_{W}-\frac{1}{d}-\frac{2 \varepsilon}{d}-\frac{1}{2} \leqslant \theta<\theta_{W}+1 .
$$

If $\tau \Psi\left(\mathcal{O}_{x}\right), \mathbb{C}(1), \cdots, \mathbb{C}(-\varepsilon)$ are $\sigma_{G}$-semi-stable for all $x \in X$, then equality (74) holds.

Proof. The argument is similar to the one in [Bri08, Section 10]. Let $\phi_{x}$ be the phase of $\tau \Psi\left(\mathcal{O}_{x}\right)$ for $x \in X$ and let $\phi_{j}$ be the phase of $\mathbb{C}(j)$ for $1 \leqslant j \leqslant-\varepsilon$. Since $\tau \Psi\left(\mathcal{O}_{x}\right)$ and $\mathbb{C}(j)$ for $1 \leqslant j \leqslant-1-\varepsilon$ are objects in $\mathcal{A}_{W}$, and $\mathcal{A}_{G}$ is obtained as a tilting of $\mathcal{A}_{W}$, the phases $\phi_{x}, \phi_{j}$ are contained in $(\theta, \theta+2]$ for $1 \leqslant j \leqslant-1-\varepsilon$. On the other hand, condition (75) implies that

$$
\theta<\theta_{W}+1<\theta_{W}+1+\frac{2}{d}<\theta_{W}+\frac{1}{d}+\frac{3}{2}<\cdots<\theta_{W}+\frac{1}{d}+\frac{2(-1-\varepsilon)}{d}+\frac{3}{2} \leqslant \theta+2 .
$$

By comparing (76) with (66), we obtain

$$
\phi_{x}=\theta_{W}+1+\frac{2}{d}, \quad \phi_{j}=\theta_{W}+\frac{1}{d}+\frac{2 j}{d}+\frac{3}{2}
$$




\section{YuKINOBU TODA}

for $1 \leqslant j \leqslant-1-\varepsilon$. We show that (77) also holds for $j=-\varepsilon$. By Lemma 3.11, we have $\mathbb{C}(-\varepsilon)[-1] \in \mathcal{A}_{W}$. Therefore if $\mathbb{C}(-\varepsilon)$ is $\sigma_{G}$-stable, then we have either

$$
\begin{array}{lll}
\mathbb{C}(-\varepsilon) \in \mathcal{A}_{G}[1], & \phi_{-\varepsilon} \in(\theta+1, \theta+2] & \text { or } \\
\mathbb{C}(-\varepsilon) \in \mathcal{A}_{G}[2], & \phi_{-\varepsilon} \in(\theta+2, \theta+3] . &
\end{array}
$$

In the case of (78), equality (77) also holds for $j=-\varepsilon$ by the inequalities (76). In the case of (79), we need to exclude the case

$$
\phi_{-\varepsilon}=\phi_{-1-\varepsilon}+2+\frac{2}{d} .
$$

If this happens, then $\phi_{-1-\varepsilon}<\theta+1$, and Lemma 3.11 and (76) imply that

$$
Z_{G}(\mathbb{C}(-\varepsilon)[-1]) \in\left\{\mathbb{R}_{>0} e^{\sqrt{-1} \pi \phi}: \phi \in(\theta, \theta+1)\right\} .
$$

This contradicts the fact that $\phi_{-\varepsilon} \in(\theta+2, \theta+3]$, hence (77) also holds for $j=-\varepsilon$.

Note that $\tau\left(\mathcal{A}_{W}\right)$ is generated by $\tau \Psi\left(\mathcal{O}_{x}\right)$ for all $x \in X$ and $\mathbb{C}(j)$ for $1 \leqslant j \leqslant-\varepsilon$, whose phases are given by (77). By inequality (75), we have

$$
\theta+\frac{2}{d} \geqslant \theta_{W}+\frac{1}{d}-\frac{2 \varepsilon}{d}+\frac{3}{2}-j=\phi_{-\varepsilon}-j
$$

for $j \geqslant 2$. Noting that there is no non-trivial homomorphism from $\mathcal{P}(\phi)$ to $\mathcal{P}\left(\phi^{\prime}\right)$ if $\phi>\phi^{\prime}$, it follows that

$$
\operatorname{Hom}^{<-1}\left(\mathcal{P}_{G}\left(\left(\theta+\frac{2}{d}, \theta+\frac{2}{d}+1\right]\right), \tau(E)\right)=0
$$

for any $E \in \mathcal{A}_{W}$. Similarly, inequality (75) implies

$$
\phi_{x}=\theta_{W}+1+\frac{2}{d}>\theta+\frac{2}{d}+1-j
$$

for $j \geqslant 1$. It follows that

$$
\operatorname{Hom}^{<0}\left(\tau(E), \mathcal{P}_{G}\left(\left(\theta+\frac{2}{d}, \theta+\frac{2}{d}+1\right]\right)\right)=0
$$

for any $E \in \mathcal{A}_{W}$. The vanishings (80), (81) imply that the right-hand side of (74) is obtained as a tilting of $\tau\left(\mathcal{A}_{W}\right)$. Hence the result follows from Lemma 4.11 below.

We have used the following lemma.

Lemma 4.11. Let $\mathcal{D}$ be a triangulated category, let $\mathcal{A} \subset \mathcal{D}$ be the heart of a bounded $t$-structure on $\mathcal{D}$, and let $Z: K(\mathcal{D}) \rightarrow \mathbb{C}$ be a group homomorphism. Suppose that there are torsion pairs $\left(\mathcal{T}_{k}, \mathcal{F}_{k}\right), k=1,2$ on $\mathcal{A}$ such that, for $\mathcal{B}_{k}=\left\langle\mathcal{F}_{k}, \mathcal{T}_{k}[-1]\right\rangle_{\text {ex }}$ the associated tilting, both of the triples

$$
\left(Z, \mathcal{B}_{1}, \theta\right), \quad\left(Z, \mathcal{B}_{2}, \theta\right)
$$

determine stability conditions. Then $\mathcal{B}_{1}=\mathcal{B}_{2}$.

Proof. We may assume $\theta=0$. Let us take an object $E \in \mathcal{T}_{1}$. Since $\left(\mathcal{T}_{2}, \mathcal{F}_{2}\right)$ is a torsion pair, there is an exact sequence

$$
0 \rightarrow F \rightarrow E \rightarrow G \rightarrow 0
$$

in $\mathcal{A}$ such that $F \in \mathcal{T}_{2}$ and $G \in \mathcal{F}_{2}$. Also, since $\left(\mathcal{T}_{1}, \mathcal{F}_{1}\right)$ is a torsion pair, objects in $\mathcal{T}_{1}$ are closed under quotients, hence $G \in \mathcal{T}_{1}$. Suppose $G \neq 0$. Then since the triples (82) determine stability conditions for $\theta=0$, it follows that $\operatorname{Im} Z(G)=0$. Hence the condition $G \in \mathcal{F}_{2}$ implies 


\section{GePNER TyPE STABILITy CONDITIONS}

$\operatorname{Re} Z(G) \in \mathbb{R}_{<0}$ but the condition $G \in \mathcal{T}_{2}$ implies $\operatorname{Re} Z(G) \in \mathbb{R}_{>0}$, which is a contradiction. Therefore $G=0$, and $\mathcal{T}_{1} \subset \mathcal{T}_{2}$ follows. Similarly, $\mathcal{T}_{2} \subset \mathcal{T}_{1}$ also holds, hence $\mathcal{T}_{1}=\mathcal{T}_{2}$ holds. Because $\mathcal{F}_{k}$ is an orthogonal complement of $\mathcal{T}_{k}$ in $\mathcal{A}$, we have $\mathcal{F}_{1}=\mathcal{F}_{2}$, hence $\mathcal{B}_{1}=\mathcal{B}_{2}$ holds.

Next we discuss the case $n=3$. In this case, we need to add an extra check of $\sigma_{G}$-stability.

Lemma 4.12. Suppose $n=3$ and that the following inequality holds:

$$
\theta_{W}-\frac{1}{d}-\frac{2 \varepsilon}{d}-\frac{1}{2} \leqslant \theta \leqslant \theta_{W}
$$

Suppose furthermore that $\tau \Psi\left(\mathcal{O}_{x}\right)$ is $\sigma_{G}$-stable, $\mathbb{C}(1), \cdots, \mathbb{C}(-\varepsilon)$ are $\sigma_{G}$-semi-stable and the following holds for all $x \in X$ :

$$
\tau^{1-\varepsilon} \Psi\left(\mathcal{O}_{x}\right) \in \mathcal{P}_{G}\left(1+\theta_{W}+\frac{2(1-\varepsilon)}{d}\right)
$$

Then equality (74) holds.

Proof. By following the proof of Lemma 4.10, we have the vanishings (80), (81) for $E=\Psi\left(\mathcal{O}_{x}\right)$, $\mathbb{C}(0), \cdots, \mathbb{C}(-1-\varepsilon)$ with $x \in X$. Below we show these vanishing also holds for $E=\Psi(F)$ for any $F \in \operatorname{Coh}(X)$. Then the right-hand side of (74) is shown to be a tilting of $\tau\left(\mathcal{A}_{W}\right)$, hence Lemma 4.11 gives the result.

Let us take an object

$$
A \in \tau^{-1} \mathcal{P}_{G}\left(\left(\theta+\frac{2}{d}, \theta+\frac{2}{d}+1\right]\right)
$$

and let

$$
\Psi^{R}: \operatorname{HMF}^{\mathrm{gr}}(W) \rightarrow D^{b} \operatorname{Coh}(X)
$$

be the right adjoint functor of $\Psi$. We claim that $\Psi^{R}(A) \in D^{b} \operatorname{Coh}(X)$ satisfies

$$
\mathcal{H}^{j}\left(\Psi^{R}(A)\right)=0 \quad \text { for } j \neq 0,1 .
$$

This property will be proved in Sublemma 4.13 below, and we continue the proof assuming this fact. Let us take the distinguished triangle

$$
\Psi \Psi^{R}(A) \rightarrow A \rightarrow B
$$

in $\operatorname{HMF}^{\mathrm{gr}}(W)$, where $B$ satisfies

$$
B \in\langle\mathbb{C}(-1-\varepsilon), \cdots, \mathbb{C}(0)\rangle .
$$

For a coherent sheaf $F$ on $X$, we apply $\operatorname{Hom}(\Psi(F), *)$ to the distinguished triangle (87). Since $B$ is right orthogonal to $\Psi D^{b} \operatorname{Coh}(X)$, and $\Psi^{R}(A)$ satisfies condition (86), we have

$$
\begin{aligned}
\operatorname{Hom}^{<0}(\tau \Psi(F), \tau(A)) & \cong \operatorname{Hom}^{<0}\left(F, \Psi^{R}(A)\right) \\
& \cong 0,
\end{aligned}
$$

which proves the vanishing (81) for $E=\Psi(F)$.

Similarly, applying $\operatorname{Hom}(*, \Psi(F))$ to the triangle $(87)$, and noting that

$$
\begin{aligned}
\operatorname{Hom}^{<-1}\left(\Psi \Psi^{R}(A), \Psi(F)\right) & \cong \operatorname{Hom}^{<-1}\left(\Psi^{R}(A), F\right) \\
& \cong 0
\end{aligned}
$$

by the property (86), we see that the vanishing (80) for $E=\Psi(F)$ is equivalent to

$$
\operatorname{Hom}^{<-1}(B, \Psi(F)) \cong 0 \text {. }
$$




\section{YuKINOBU TODA}

To show (88), note that the vanishing (80) for $E=\mathbb{C}(j), 0 \leqslant j \leqslant-1-\varepsilon$ and the triangle (87) imply

$$
\operatorname{Hom}^{<-1}(B, \mathbb{C}(j)) \cong 0, \quad j=0, \cdots,-1-\varepsilon .
$$

Therefore, if we denote by $\mathcal{H}_{\mathcal{A}_{W}}^{i}(B) \in \mathcal{A}_{W}$ the $i$ th cohomology with respect to the t-structure on $\operatorname{HMF}^{\mathrm{gr}}(W)$ with heart $\mathcal{A}_{W}$, then we have $\mathcal{H}_{\mathcal{A}_{W}}^{i}(B)=0$ for $i>1$. Since $\Psi(F) \in \mathcal{A}_{W}$, this implies that (88) holds. Therefore the vanishing (80) for $E=\Psi(F)$ holds.

We have used the following sublemma.

Sublemma 4.13. Condition (86) holds.

Proof. We investigate the vanishing

$$
\operatorname{Hom}^{j}\left(\Psi^{R}(A), \mathcal{O}_{x}\right)=0
$$

for $x \in X$ and $j \in \mathbb{Z}$. By the Serre duality on $X$, adjunction and applying $\tau$, the vanishing (89) is equivalent to

$$
\operatorname{Hom}\left(\tau \Psi\left(\mathcal{O}_{x}\right)[j-1], \tau(A)\right)=0 .
$$

We first show that (90) holds for any $j<-1$ and $x \in X$. Applying the Serre functor $\mathcal{S}_{W}=\tau^{-\varepsilon}[1]$ on $\operatorname{HMF}^{\text {gr }}(W)$, the vanishing (90) is equivalent to

$$
\operatorname{Hom}\left(\tau(A)[-j], \tau^{1-\varepsilon} \Psi\left(\mathcal{O}_{x}\right)\right)=0 .
$$

On the other hand, by assumption (83), we have the inequality

$$
\theta+\frac{2}{d}-j \geqslant 1+\theta_{W}+\frac{2(1-\varepsilon)}{d}
$$

for $j<-1$. Therefore by our assumptions (84) and (85), the vanishing (91) holds for $j<-1$.

Next, we have the inequality

$$
\theta_{W}+1+\frac{2}{d}+j-1>\theta+\frac{2}{d}+1
$$

for $j \geqslant 2$ by (83), hence the vanishing (90) holds for $j \geqslant 2$. Moreover, the above inequality, hence the vanishing (90), also holds for $j=1$ unless $\theta=\theta_{W}$. Suppose $\theta=\theta_{W}$, and let $P$ be the $\sigma_{G}$-Harder-Narasimhan factor of $\tau(A)$ with the maximum phase. Note that $\tau \Psi\left(\mathcal{O}_{x}\right)$ is $\sigma_{G}$-stable with phase $\theta_{W}+1+2 / d$, which is bigger than or equal to the phase of $P$. Therefore the vanishing (90) for $j=1$ holds unless we have

$$
P \in \mathcal{P}_{G}\left(\theta_{W}+1+\frac{2}{d}\right)
$$

and $\tau \Psi\left(\mathcal{O}_{x}\right)$ is one of the Jordan-Hölder factors of $P$. It follows that, by taking the Jordan-Hölder filtration of $P$, there is a distinguished triangle

$$
\tau \Psi(Q) \rightarrow \tau(A) \rightarrow \tau\left(A^{\prime}\right)
$$

where $Q$ is a zero-dimensional coherent sheaf on $X$, and $A^{\prime}$ is an object in the right-hand side of (85), such that the vanishing (90) holds for $j \geqslant 1$ after replacing $A$ by $A^{\prime}$.

Applying $\Psi^{R} \circ \tau^{-1}$ to (92), we have the distinguished triangle

$$
Q \rightarrow \Psi^{R}(A) \rightarrow \Psi^{R}\left(A^{\prime}\right) .
$$

The argument above shows that, after replacing $A$ by $A^{\prime}$, the vanishing (89) holds unless $j=$ $-1,0$. It follows that $\Psi^{R}\left(A^{\prime}\right)$ is a two-term complex of vector bundles on $X$ whose cohomology 


\section{GEPNER TYPE STABILITY CONDITIONS}

is concentrated in degrees 0 and 1. Since $Q \in \operatorname{Coh}(X)$, we conclude that $\mathcal{H}^{j}\left(\Psi^{R}(A)\right)=0$ for $j \neq 0,1$.

By the results above, the problem is reduced to showing the $\sigma_{G}$-stability of some objects in $\operatorname{HMF}^{\text {gr }}(W)$. The following lemma is useful in checking the $\sigma_{G}$-stability of these objects. The proof is obvious, and we omit it.

LEMma 4.14. Let $\mathcal{D}$ be a triangulated category, $Z: K(\mathcal{D}) \rightarrow \mathbb{C}$ a group homomorphism and $\mathcal{A} \subset \mathcal{D}$ the heart of a bounded $t$-structure on $\mathcal{D}$. Suppose that the triple $(Z, \mathcal{A}, \theta=0)$ determines a stability condition on $\mathcal{D}$, and the following condition holds:

$$
c_{\min }:=\inf \{\operatorname{Im} Z(E)>0: E \in \mathcal{A}\}>0 .
$$

Then an object $E \in \mathcal{A}$ with $\operatorname{Im} Z(E)=c_{\text {min }}$ is $\sigma_{G}$-stable if and only if $\operatorname{Hom}(P, E)=0$ for any $P \in \mathcal{A}$ with $\operatorname{Im} Z(P)=0$.

\section{Proof of Theorem 1.3}

In this section, we apply the strategy in the previous section and prove Theorem 1.3. Below we use the same notation as in the previous section. In particular, the constants $C_{W} \in \mathbb{C}^{*}$ and $\theta_{W} \in \mathbb{Q}$ are defined as in Lemma 4.3, in both the $\varepsilon=0$ and the $\varepsilon<0$ case. The goal is to prove Conjecture 2.9 when

$$
n-4 \leqslant \varepsilon \leqslant 0
$$

and $X$ does not contain stacky points. Since we already discussed the case with $n=1$ and $n=2$, $\varepsilon=0$, the following five possibilities are left:

$$
(n, \varepsilon)=(3,0),(2,-1),(4,0),(3,-1),(2,-2) .
$$

We divide the proof into five subsections, where each subsection corresponds to one of these types. We repeat similar arguments in these subsections, so we recommend the readers to follow only one or two cases, for example $(n, \varepsilon)=(4,0)$ or $(3,-1)$, at the first reading of this paper.

\subsection{The case $n=3, \varepsilon=0$}

In this subsection, we study the case $n=3$ and $\varepsilon=0$. In this case, $X$ is a smooth elliptic curve, and the heart $\mathcal{A}_{W}$ is given by

$$
\mathcal{A}_{W}=\Psi \operatorname{Coh}(X) .
$$

Furthermore, possible data $\left(a_{1}, a_{2}, a_{3}, d, \int_{X} H\right)$ are classified into three types [Sai87, Table 2]

$$
\left(a_{1}, a_{2}, a_{3}, d, \int_{X} H\right)=\left\{\begin{array}{l}
(1,1,1,3,3) \\
(2,1,1,4,2) \\
(3,2,1,6,1) .
\end{array}\right.
$$

The central charge $Z_{G}$ can be described as follows.

Lemma 5.1. Suppose $n=3$ and $\varepsilon=0$. For any $E \in D^{b} \operatorname{Coh}(X)$, we have

$$
Z_{G}(\Psi(E))=C_{W}\left\{-d(E)+r(E)\left(\cos \frac{2 \pi}{d}-1\right)+r(E) \sin \frac{2 \pi}{d} \sqrt{-1}\right\},
$$

where $(r(E), d(E))=(\operatorname{rank}(E), \operatorname{deg}(E))$. 


\section{YukinOBU TODA}

Proof. Equation (63) becomes

$$
\left(\alpha_{0}^{\dagger},-1\right)\left(\begin{array}{cc}
1-e^{2 \pi \sqrt{-1} / d}-\int_{X} H & -1 \\
H & 1-e^{2 \pi \sqrt{-1} / d}
\end{array}\right)=0,
$$

giving $\int_{X} \alpha_{0}^{\dagger}=e^{2 \pi \sqrt{-1} / d}-1$.

We set the slope function $\mu$ in (67) to be the constant function $\mu=-1$, so that the resulting heart $\mathcal{A}_{G}$ in (69) coincides with $\mathcal{A}_{W}$. We have the following result.

Proposition 5.2. Suppose $n=3$ and $\varepsilon=0$. Then the triple

$$
\left(Z_{G}, \mathcal{A}_{W}, \theta=\theta_{W}\right)
$$

determines a Gepner type stability condition $\sigma_{G}$ on $\operatorname{HMF}^{\mathrm{gr}}(W)$ with respect to $(\tau, 2 / d)$.

Proof. Note that, since $X$ is an elliptic curve, the space of stability conditions on $D^{b} \operatorname{Coh}(X)$ is completely described in [Bri07, Section 9]. Since $\mathcal{A}_{W}=\Psi \operatorname{Coh}(X)$, and the central charge $Z_{G}$ is given by (94), it follows that the triple (95) satisfies condition (71). Then the same argument of [Bri07, Example 5.4] shows that the triple (95) determines a stability condition $\sigma_{G}$ on $\operatorname{HMF}^{\mathrm{gr}}(W)$. Now we are going to apply Lemma 4.12. Note that inequality (83) is satisfied in this case. For a point $x \in X$, we have

$$
\tau \Psi\left(\mathcal{O}_{x}\right) \cong \Psi\left(\mathcal{O}_{X}(-x)[1]\right)
$$

by Proposition 3.2. Since this object is $\sigma_{G}$-stable with phase $1+\theta_{W}+2 / d$, Lemma 4.12 implies that $\sigma_{G}$ is a Gepner type stability condition with respect to $(\tau, 2 / d)$.

5.2 The case $n=2, \varepsilon=-1$

In this subsection, we study the case $n=2, \varepsilon=-1$. In this case, $X$ is a finite number of smooth points, and the heart $\mathcal{A}_{W}$ is given by

$$
\mathcal{A}_{W}=\left\langle\mathbb{C}(0), \Psi\left(\mathcal{O}_{x}\right): x \in X\right\rangle_{\mathrm{ex}} .
$$

The following lemma immediately follows from (66).

Lemma 5.3. If we write the K-theory class of an object $E \in \mathcal{A}_{W}$ as

$$
[E]=v_{0}[\mathbb{C}(0)]+\sum_{x \in X} w_{x}\left[\Psi\left(\mathcal{O}_{x}\right)\right]
$$

and set $w:=\sum_{x \in X} w_{x}$, then $Z_{G}(E)$ is given by

$$
Z_{G}(E)=C_{W}\left\{-w+v_{0}\left(1-\cos \frac{2 \pi}{d}\right)-v_{0} \sin \frac{2 \pi}{d} \sqrt{-1}\right\} .
$$

In the notation used in Subsection 4.2, the polynomial $\widehat{W}$ is of type $\left(a_{1}, a_{2}, 1, d\right)$, which must belong to one of the classifications in (93). Furthermore, applying Lemma 3.17 and Lemma 3.18, we see that $\mathcal{A}_{W}$ is equivalent to the abelian category of representations of a certain quiver $\mathcal{Q}$. 


\section{GePNER TyPE STABILITy CONDITIONS}

By these arguments, the possible types of $\left(a_{1}, a_{2}, d, \sharp X, \mathcal{Q}\right)$ can be classified as follows:

$$
\left(a_{1}, a_{2}, d, \sharp X\right)=(1,1,3,3), \quad \mathcal{Q}=
$$

Here the left vertex of the quiver $\mathcal{Q}$ corresponds to $\mathbb{C}(0)$, and the right vertices correspond to $\Psi\left(\mathcal{O}_{x}\right)$ for $x \in X$. In all these cases, we set $\mu=-1$ so that the heart (69) coincides with $\mathcal{A}_{W}$. We have the following result.

Proposition 5.4. Suppose that $n=2$ and $\varepsilon=-1$. Then the triple

$$
\left(Z_{G}, \mathcal{A}_{W}, \theta\right), \quad \frac{5}{6}+\theta_{W} \leqslant \theta<1+\theta_{W}
$$

determines a Gepner type stability condition $\sigma_{G}$ on $\operatorname{HMF}^{\mathrm{gr}}(W)$ with respect to $(\tau, 2 / d)$.

Proof. By Lemma 5.3, it follows that the triple (96) satisfies condition (71). Since $\mathcal{A}_{W}$ is noetherian and artinian, the triple (96) satisfies the Harder-Narasimhan property (cf. [Bri07, Proposition 2.4]), hence it determines a stability condition $\sigma_{G}$ on $\operatorname{HMF}^{\mathrm{gr}}(W)$. In order to show the Gepner type property of $\sigma_{G}$, it is enough to check the assumption of Lemma 4.10. Since inequality (75) is satisfied, it remains to show the $\sigma_{G}$-stability of $\tau \Psi\left(\mathcal{O}_{x}\right)$ and $\mathbb{C}(1)$ for all $x \in X$, which we prove in the next lemma.

Lemma 5.5. The objects $\tau \Psi\left(\mathcal{O}_{x}\right)$ and $\mathbb{C}(1)$ are $\sigma_{G \text {-stable. }}$

Proof. The object $\tau \Psi\left(\mathcal{O}_{x}\right)$ fits into a unique non-trivial extension in $\mathcal{A}_{W}$ by Lemma 3.10 and Remark 3.13:

$$
0 \rightarrow \Psi\left(\mathcal{O}_{x}\right) \rightarrow \tau \Psi\left(\mathcal{O}_{x}\right) \rightarrow \mathbb{C}(0) \rightarrow 0
$$

Therefore, $\Psi\left(\mathcal{O}_{x}\right)$ is the only non-trivial subobject of $\tau \Psi\left(\mathcal{O}_{x}\right)$ in $\mathcal{A}_{W}$. Comparing the argument of $Z_{G}(*)$, we see that $\tau \Psi\left(\mathcal{O}_{x}\right)$ is $Z_{G \text {-stable. }}$

For $\mathbb{C}(1)$, note that the object $\mathbb{C}(1)[-1]$ is contained in $\mathcal{A}_{W}$ by Lemma 3.11 . Let $0 \neq F \in \mathcal{A}_{W}$ be a proper subobject of $\mathbb{C}(1)[-1]$ in $\mathcal{A}_{W}$. As in Corollary 3.20, the inclusion $F \subset \mathbb{C}(1)[-1]$ can 


\section{YuKINOBU TODA}

be represented by the following inclusion of quiver representations:

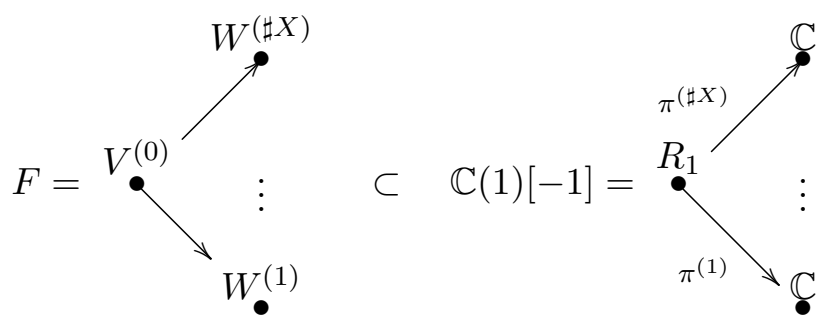

Here $R_{1}$ is the space of degree one elements in $R=\mathbb{C}\left[x_{1}, x_{2}\right]$, whose dimension is $\sharp X-1$. The morphisms $\pi^{(i)}: R_{1} \rightarrow \mathbb{C}$ are evaluations at the points in $X$, and $V^{(0)}, W^{(i)}$ are finite-dimensional vector spaces with $V^{(0)} \subset R_{1}$, $\operatorname{dim} W^{(i)} \leqslant 1$. Let $I$ be the subset of $i \in\{1, \cdots, \sharp X\}$ satisfying $W^{(i)}=0$. Then we have

$$
V^{(0)} \subset \bigcap_{i \in I} \operatorname{Ker}\left(\pi^{(i)}\right)
$$

hence $\operatorname{dim} V^{(0)} \leqslant \sharp X-1-|I|$. Since we have

$$
\begin{aligned}
Z_{G}(\mathbb{C}(1)[-1]) & =C_{W}\left\{-\sharp X+(\sharp X-1) \cdot\left(1-e^{2 \pi \sqrt{-1} / d}\right)\right\} \\
Z_{G}(F) & =C_{W}\left\{|I|-\sharp X+\operatorname{dim} V^{(0)} \cdot\left(1-e^{2 \pi \sqrt{-1} / d}\right)\right\}
\end{aligned}
$$

it follows that the argument of $Z_{G}(F)$ is less than that of $Z_{G}(\mathbb{C}(1)[-1])$ in $(\pi \theta, \pi \theta+\pi]$. Therefore $\mathbb{C}(1)[-1]$ is $\sigma_{G}$-stable.

5.3 The case $n=4, \varepsilon=0$

In this subsection, we study the case of $(n, \varepsilon)=(4,0)$. In this case, $X$ is a smooth projective $\mathrm{K} 3$ surface, and the heart $\mathcal{A}_{W}$ is given by

$$
\mathcal{A}_{W}=\Psi \operatorname{Coh}(X) .
$$

By Theorem 3.1, the triangulated category $\operatorname{HMF}^{\mathrm{gr}}(W)$ is equivalent to $D^{b} \mathrm{Coh}(X)$ via the equivalence $\Psi$. On the other hand, the spaces of stability conditions on K3 surfaces have been studied by Bridgeland [Bri08]. Combining the techniques in [Bri08] with the arguments in Subsection 4.3, we construct a desired Gepner type stability condition on $\operatorname{HMF}^{\text {gr }}(W)$.

Let us first describe the matrix $M$ which appears in equation (58). By writing $\int_{X} H^{2}=2 m$ for $m \in \mathbb{Z}_{\geqslant 1}$, the matrix $M$ is given by

$$
M=\left(\begin{array}{ccc}
-1-m & -H & -1 \\
H & 1 & 0 \\
m & H & 1
\end{array}\right) .
$$

By using this description, we give a classification of possible types.

Lemma 5.6. If $n=4$, the possible data $\left(a_{1}, a_{2}, a_{3}, a_{4}, d, \int_{X} H^{2}\right)$ can be classified into the two types:

$$
\left(a_{1}, a_{2}, a_{3}, a_{4}, d, \int_{X} H^{2}\right)=\left\{\begin{array}{l}
(1,1,1,1,4,4) \\
(3,1,1,1,6,2)
\end{array} .\right.
$$

Proof. By (97), we have

$$
\operatorname{det}(M-\lambda \cdot \mathrm{id})=-(\lambda+1)\left\{\lambda^{2}+(m-2) \lambda+1\right\} .
$$




\section{GePNER TyPE STABILITy CONDITIONS}

Since $\operatorname{det}(M-\lambda \cdot \mathrm{id})=0$ for $\lambda=e^{ \pm 2 \pi \sqrt{-1} / d}$, we have

$$
2 \cos (2 \pi / d)=m-2 .
$$

This is only possible for $(m, d)=(2,4),(1,6)$. In the latter case, the possibility $\left(a_{1}, a_{2}, a_{3}, a_{4}\right)=$ $(2,2,1,1)$ is excluded since the stack $X$ always contains a stacky point. Therefore we obtain the classification (98).

Remark 5.7. In the type $(1,1,1,1,4,4)$ case, $X$ is a quartic K3 surface. In the type $(3,1,1,1,6,2)$ case, $X$ is a double cover of $\mathbb{P}^{2}$.

In order to describe the central charge $Z_{G}$, it is convenient to use the twisted Mukai vector. For $B \in H^{2}(X, \mathbb{R})$, we set

$$
v^{B}(E):=e^{-B} \operatorname{ch}(E) \sqrt{\operatorname{td}_{X}} .
$$

We denote by $v_{i}^{B}(E)$ the $H^{i, i}(X)$-component of $v^{B}(E)$. The central charge $Z_{G}$ is computed in the following way.

Lemma 5.8. By setting $B=-H / 2$, the following holds for $E \in D^{b} \operatorname{Coh}(X)$ :

$$
Z_{G}(\Psi(E))=C_{W}\left(-v_{2}^{B}(E)+\frac{d}{8} v_{0}^{B}(E)+\frac{1}{2} \sqrt{\frac{d}{H^{2}}} H \cdot v_{1}^{B}(E) \sqrt{-1}\right) .
$$

Proof. By (97), equation (63) becomes

$$
\left(\alpha_{0}^{\dagger}, \alpha_{1}^{\dagger},-1\right)\left(\begin{array}{ccc}
-1-m-e^{2 \pi \sqrt{-1} / d} & -H & -1 \\
H & 1-e^{2 \pi \sqrt{-1} / d} & 0 \\
m & H & 1-e^{2 \pi \sqrt{-1} / d}
\end{array}\right)=0,
$$

which gives

$$
\int_{X} \alpha_{0}^{\dagger}=e^{2 \pi \sqrt{-1} / d}-1, \quad \alpha_{1}^{\dagger}=\frac{e^{2 \pi \sqrt{-1} / d}}{1-e^{2 \pi \sqrt{-1} / d}} H .
$$

Applying the classification in Lemma 5.6, a straightforward computation shows the result.

Remark 5.9. Equality (100) can be written as the integral

$$
Z_{G}(\Psi(E))=-C_{W} \int_{X} e^{-\sqrt{-1} \omega} v^{-H / 2}(E)
$$

where $\omega \in \mathbb{R}_{>0} H$ satisfies $\int_{X} \omega^{2}=d / 4$.

By setting $B=-H / 2$, we consider the slope function $\mu$ on $\mathcal{A}_{W}=\Psi \operatorname{Coh}(X)$ defined by

$$
\mu(\Psi(E))=\frac{v_{1}^{B}(E) \cdot H}{v_{0}^{B}(E)} .
$$

Here $\mu(\Psi(E))$ is defined to be $\infty$ if $E$ is a torsion sheaf. This $\mu$-stability coincides with the classical slope stability condition on $\operatorname{Coh}(X)$ via $\Psi$. As we discussed in Subsection 4.3, the slope function $\mu$ defines a torsion pair (68) on $\mathcal{A}_{W}$, and the associated tilting $\mathcal{A}_{G}$ is given by (69). We have the following result.

Proposition 5.10. Suppose that $n=4$ and $\varepsilon=0$. Then the triple

$$
\left(Z_{G}, \mathcal{A}_{G}, \theta=\theta_{W}-1\right)
$$

determines a Gepner type stability condition $\sigma_{G}$ on $\operatorname{HMF}^{\mathrm{gr}}(W)$ with respect to $(\tau, 2 / d)$. 


\section{YUKINOBU TODA}

Proof. Instead of the triple (102), we consider the triple

$$
\left(Z_{G}^{\dagger}:=Z_{G} / C_{W}, \mathcal{A}_{G}[1], \theta=0\right) .
$$

Obviously, the triple (102) determines a stability condition if and only if the triple (103) determines a stability condition. If the latter holds, then by Remark 5.9, the resulting stability condition is $\Psi_{*}$ of one of stability conditions on $D^{b} \operatorname{Coh}(X)$ constructed in [Bri08, Section 6]. Therefore, applying [Bri08, Lemma 6.2], it is enough to show the following: for any spherical torsion free sheaf $E$ on $X$, one has $Z_{G}^{\dagger}(\Psi(E)) \notin \mathbb{R}_{\leqslant 0}$. The proof of this fact requires some more arguments, so we leave the proof to Lemma 5.11 below. We continue the proof assuming this fact.

By the argument above and Lemma 5.11, the triple (102) determines a stability condition $\sigma_{G}$ on $\operatorname{HMF}^{\mathrm{gr}}(W)$. It remains to show that $\sigma_{G}$ is of Gepner type with respect to $(\tau, 2 / d)$. To show this, let us consider the objects $\tau \Psi\left(\mathcal{O}_{x}[-1]\right)$ for $x \in X$. By Proposition 3.2, we have

$$
\tau \Psi\left(\mathcal{O}_{x}[-1]\right) \cong \Psi\left(I_{x}\right) \in \mathcal{A}_{G}[1]
$$

where $I_{x} \subset \mathcal{O}_{X}$ is the ideal sheaf which defines $x$. In Lemma 5.12 below, we see that $\Psi\left(I_{x}\right)$ is $\sigma_{G}$-stable, hence it lies in $\mathcal{P}_{G}\left(\theta_{W}+2 / d\right)$. If we set $\sigma_{G}^{\prime}$ to be the stability condition defined as in (73), this implies that

$$
\Psi\left(\mathcal{O}_{x}[-1]\right) \in \tau^{-1} \mathcal{P}_{G}\left(\theta_{W}+\frac{2}{d}\right)=\mathcal{P}_{G}^{\prime}\left(\theta_{W}\right)
$$

for any $x \in X$, and that it is $\sigma_{G}^{\prime}$-stable. Since $X$ is a K3 surface, this implies that $\mathcal{P}_{G}^{\prime}\left(\left(\theta_{W}-1, \theta_{W}\right]\right)$ is obtained as a tilting of $\mathcal{A}_{W}=\Psi(\operatorname{Coh}(X))$, by the argument of [Bri08, Lemma 10.1]. Therefore $\mathcal{P}_{G}^{\prime}\left(\left(\theta_{W}-1, \theta_{W}\right]\right)$ coincides with $\mathcal{A}_{G}$ by Lemma 4.11 , which shows that $\sigma_{G}$ is of Gepner type with respect to $(\tau, 2 / d)$.

Lemma 5.11. For any spherical torsion free sheaf $E$ on $X$, one has $Z_{G}^{\dagger}(\Psi(E)) \notin \mathbb{R}_{\leqslant 0}$.

Proof. This is equivalent to saying that, for any spherical torsion free sheaf $E$ on $X$ with $v_{1}^{B}(E)$. $H=0$, one has

$$
-v_{2}^{B}(E)+\frac{d}{8} v_{0}^{B}(E)>0
$$

Since $E$ is a spherical sheaf, we have $v^{B}(E)^{2}=-2$, where the square is defined in the Mukai lattice of $X$ (cf. [Bri08, Lemma 5.1]). Combining this with $v_{1}^{B}(E) \cdot H=0$ and the Hodge index theorem, we have

$$
0 \geqslant v_{1}^{B}(E)^{2}=2 v_{0}^{B}(E) v_{2}^{B}(E)-2 .
$$

Noting that $v_{0}^{B}(E)>0$, we obtain $v_{2}^{B}(E) \leqslant 1 / v_{0}^{B}(E)$, hence

$$
-v_{2}^{B}(E)+\frac{d}{8} v_{0}^{B}(E) \geqslant \frac{1}{v_{0}^{B}(E)}\left\{\frac{d}{8} v_{0}^{B}(E)^{2}-1\right\} .
$$

The right-hand side of $(105)$ is positive if $v_{0}^{B}(E) \geqslant 2$. Therefore we may assume that $v_{0}^{B}(E)=1$; that is, $E$ is rank one. Since $E$ is a spherical sheaf, this implies that $E$ is a line bundle.

Let $l$ be the first Chern class of $E$. Since $v_{1}^{B}(E) \cdot H=0$, we have $l \cdot H=-H^{2} / 2$, and $v_{2}^{B}(E)$ can be written as

$$
v_{2}^{B}(E)=\frac{l^{2}}{2}-\frac{H^{2}}{8}+1
$$

Suppose that (104) does not hold for a line bundle $E$. Then, combining with the Hodge index 


\section{GEPNER TYPE STABILITY CONDITIONS}

theorem, we obtain

$$
\frac{H^{2}}{4}-2+\frac{d}{4} \leqslant l^{2} \leqslant \frac{H^{2}}{4} .
$$

Since we have only two cases, $\left(d, H^{2}\right)=(4,4),(6,2)$, and $l^{2}$ is an even integer, it follows that $l^{2}=0$. Also since $-l \cdot H$ is either 2 (when $X$ is a quartic K3 surface) or 1 (when $X$ is a double cover of $\mathbb{P}^{2}$ ), it follows that the linear system $|-l|$ defines an elliptic fibration $X \rightarrow \mathbb{P}^{1}$. In particular, its general fiber is a smooth elliptic curve $C \in|-l|$ in $X$. However, this gives a contradiction: when $X$ is a quartic K3 surface, we have the Castelnuovo inequality

$$
g(C) \leqslant \frac{1}{2}(H \cdot C-1)(H \cdot C-2)=0
$$

which contradicts $g(C)=1$. When $X$ is a double cover of $\mathbb{P}^{2}$, if $\pi: X \rightarrow \mathbb{P}^{2}$ is the double cover, then $H \cdot C=1$ implies that $\left.\pi\right|_{C}$ is an isomorphism between $C$ and a line in $\mathbb{P}^{2}$, which contradicts the fact that $C$ is an elliptic curve. Therefore (104) also holds for a line bundle $E$.

Lemma 5.12. The object $\Psi\left(I_{x}\right) \in \mathcal{A}_{G}[1]$ is $\sigma_{G}$-stable.

Proof. It is enough to show the $Z_{G}^{\dagger}$-stability of $\Psi\left(I_{x}\right)$ with respect to the triple (103). By Lemma 5.8, we have the following:

$$
\operatorname{Im} Z_{G}^{\dagger}\left(\mathcal{A}_{G}[1]\right) \subset\left\{\frac{1}{2} \sqrt{\frac{d}{H^{2}}} \times \mathbb{Z}_{\geqslant 0}\right\} .
$$

We first consider the case $\left(d, H^{2}\right)=(6,2)$. In this case, the imaginary part of $Z_{G}^{\dagger}\left(\Psi\left(I_{x}\right)\right)$ is $\sqrt{3} / 2$; that is, the smallest positive value of the right-hand side of (106). Therefore, by Lemma 4.14, the $Z_{G}^{\dagger}$-stability of $\Psi\left(I_{x}\right)$ follows from

$$
\operatorname{Hom}\left(P, \Psi\left(I_{x}\right)\right)=0
$$

for any $P \in \mathcal{A}_{G}[1]$ with $\operatorname{Im} Z_{G}^{\dagger}(P)=0$. This vanishing holds since $\Psi^{-1}(P)$ is given by an iterated extensions of zero dimensional sheaves and objects $U[1]$ for torsion free $\mu$-stable sheaves with $\mu(U)=0$.

Next we consider the case $\left(d, H^{2}\right)=(4,4)$. In this case, $Z_{G}^{\dagger}\left(\Psi\left(I_{x}\right)\right)=\sqrt{-1}$, whose imaginary part is the twice of the smallest positive value $1 / 2$ of the right-hand side of (106). Therefore, besides the vanishing (107), we need to show the following: if there is an exact sequence

$$
0 \rightarrow P_{1} \rightarrow \Psi\left(I_{x}\right) \rightarrow P_{2} \rightarrow 0
$$

in $\mathcal{A}_{G}[1]$ with $\operatorname{Im} Z_{G}^{\dagger}\left(P_{i}\right)=1 / 2$, then we have the inequality

$$
\arg Z_{G}^{\dagger}\left(P_{1}\right)<\arg Z_{G}^{\dagger}\left(\Psi\left(I_{x}\right)\right)=\frac{\pi}{2}
$$

in $(0, \pi]$. In order to show this, we first observe that $\Psi\left(\mathcal{O}_{X}\right) \in \mathcal{A}_{G}[1]$ is $Z_{G}^{\dagger}$-stable by [BMT14, Proposition 7.4.1]. Since $\Psi\left(I_{x}\right)$ is a subobject of $\Psi\left(\mathcal{O}_{X}\right)$ in $\mathcal{A}_{G}[1]$ and $Z_{G}^{\dagger}\left(\Psi\left(\mathcal{O}_{X}\right)\right)=-1+\sqrt{-1}$, we have

$$
\arg Z_{G}^{\dagger}\left(P_{1}\right)<\arg Z_{G}^{\dagger}\left(\Psi\left(\mathcal{O}_{X}\right)\right)=\frac{3}{4} \pi
$$

Since the image of $Z_{G}^{\dagger}$ is contained in $\frac{1}{2} \mathbb{Z}+\frac{\sqrt{-1}}{2} \mathbb{Z}$, and $\operatorname{Im} Z_{G}^{\dagger}\left(P_{1}\right)=1 / 2$, this inequality implies that $\arg Z_{G}^{\dagger}\left(P_{1}\right) \leqslant \pi / 2$. It remains to exclude the following case:

$$
Z_{G}^{\dagger}\left(P_{1}\right)=Z_{G}^{\dagger}\left(P_{2}\right)=\frac{\sqrt{-1}}{2} .
$$




\section{YUKINOBU TODA}

Indeed, we see that there is no object $P \in \mathcal{A}_{G}[1]$ such that $Z_{G}^{\dagger}(P)=\sqrt{-1} / 2$. Suppose that such an object $P$ exists, and let

$$
\operatorname{ch}\left(\Psi^{-1} P\right) \sqrt{\operatorname{td}_{X}}=(r, l, s) \in H^{0}(X) \oplus H^{2}(X) \oplus H^{4}(X)
$$

be the (untwisted) Mukai vector of $\Psi^{-1} P$. Then the condition $Z_{G}^{\dagger}(P)=\sqrt{-1} / 2$ is equivalent to

$$
l \cdot H+2 r=1, \quad l \cdot H+2 s=0 .
$$

This is a contradiction since both of $r$ and $s$ are integers.

5.4 The case $n=3, \varepsilon=-1$

In this subsection, we study the case $(n, \varepsilon)=(3,-1)$. This case seems to be the most interesting case studied in this paper, as a construction of $\sigma_{G}$ has to do with the study of coherent systems on the smooth projective curve $X$. Indeed, in Proposition 3.22, we constructed an equivalence $\Theta$ between the category of coherent systems on $X$ and the heart $\mathcal{A}_{W}$, given by

$$
\mathcal{A}_{W}=\langle\mathbb{C}(0), \Psi \operatorname{Coh}(X)\rangle_{\mathrm{ex}} .
$$

Below we leave out $\Theta$ and regard any coherent system $\left(\mathcal{O}_{X}^{\oplus R} \rightarrow F\right)$ on $X$ as an object in $\mathcal{A}_{W}$.

In the notation of Subsection 4.2 , the polynomial $\widehat{W}$ is of type $\left(a_{1}, a_{2}, a_{3}, 1, d\right)$, which must belong to one of the classifications (98). There are two possibilities:

$$
\left(a_{1}, a_{2}, a_{3}, d\right)= \begin{cases}(1,1,1,4), & X \text { is a genus } 3 \text { curve } \\ (3,1,1,6), & X \text { is a genus } 2 \text { curve. }\end{cases}
$$

The Calabi-Yau manifold $\widehat{X}$ is a K3 surface which is either a quartic surface or a double cover of $\mathbb{P}^{2}$, and it contains $X$ as an element in the linear system $X \in|\widehat{H}|$. The central charge $Z_{G}$ is described in terms of coherent systems as follows:

Lemma 5.13. For any coherent system $\left(\mathcal{O}_{X}^{\oplus R} \rightarrow F\right)$ on $X$, we have

$$
Z_{G}\left(\mathcal{O}_{X}^{\oplus R} \rightarrow F\right)=C_{W}\left\{-d(F)+R\left(1-\cos \frac{2 \pi}{d}\right)+\frac{\sqrt{\widehat{H}^{2} d}}{2}\left(r(F)-\frac{R}{2}\right) \sqrt{-1}\right\},
$$

where $(r(F), d(F))=(\operatorname{rank}(F), \operatorname{deg}(F))$.

Proof. The result follows from (65), (66), Lemma 5.8 and an easy computation.

By setting $Z_{G}^{\dagger}:=Z_{G} / C_{W}$, we define the slope function $\mu$ on $\mathcal{A}_{W}$ by

$$
\begin{aligned}
\mu\left(\mathcal{O}_{X}^{\oplus R} \rightarrow F\right) & :=\frac{-\operatorname{Im} Z_{G}^{\dagger}\left(\mathcal{O}_{X}^{\oplus R} \rightarrow F\right)}{R} \\
& =\frac{\sqrt{\hat{H}^{2} d}}{2} \cdot\left(\frac{1}{2}-\frac{r(F)}{R}\right) .
\end{aligned}
$$

We set $\mu(*)=-\infty$ if $R=0$.

Lemma 5.14. Any object in $\mathcal{A}_{W}$ admits a Harder-Narasimhan filtration with respect to $\mu$ stability.

Proof. Although $\mathcal{A}_{W}$ is a noetherian abelian category, we have to take a little care since the condition in [Tod10a, Proposition 2.12] is not satisfied in this case. Instead, we apply the argument used in [Tod09, Theorem 2.29]. Let $\mathcal{C} \subset \mathcal{A}_{W}$ be the subcategory consisting of objects $\left(\mathcal{O}_{X}^{\oplus R} \stackrel{s}{\rightarrow} F\right)$ 


\section{GEPNER TYPE STABILITY CONDITIONS}

such that $s$ is surjective. Note that the right orthogonal complement $\mathcal{C}^{\perp}$ consists of objects of the form $\left(0 \rightarrow F^{\prime}\right)$. Any object $\left(\mathcal{O}_{X}^{\oplus R} \stackrel{s}{\rightarrow} F\right) \in \mathcal{A}_{W}$ fits into the exact sequence

$$
0 \rightarrow\left(\mathcal{O}_{X}^{\oplus R} \stackrel{s}{\rightarrow} \operatorname{Im} s\right) \rightarrow\left(\mathcal{O}_{X}^{\oplus R} \stackrel{s}{\rightarrow} F\right) \rightarrow(0 \rightarrow \operatorname{Cok}(s)) \rightarrow 0,
$$

showing that $\left(\mathcal{C}, \mathcal{C}^{\perp}\right)$ is a torsion pair on $\mathcal{A}_{W}$. Furthermore, since $\mu(*)=-\infty$ on $\mathcal{C}^{\perp}$, we can easily see the following: an object $E \in \mathcal{C}$ is $\mu$-semi-stable if and only if for any exact sequence

$$
0 \rightarrow E_{1} \rightarrow E \rightarrow E_{2} \rightarrow 0
$$

in $\mathcal{C}$, we have $\mu\left(E_{1}\right) \leqslant \mu\left(E_{2}\right)$ (cf. [Tod09, Lemma 2.27]). Since $\mathcal{C}$ is a noetherian and artinian quasi-abelian category, an argument similar to [Tod09, Theorem 2.29] shows that any object in $\mathcal{C}$ admits a $\mu$-Harder-Narasimhan filtration. Combining this with (109), we conclude that any object in $\mathcal{A}_{W}$ admits a $\mu$-Harder-Narasimhan filtration.

Remark 5.15. By the proof of Lemma 5.14, we see that if an object $\left(\mathcal{O}_{X}^{\oplus R} \stackrel{s}{\rightarrow} F\right)$ is $\mu$-semi-stable, then either $s$ is surjective or $R=0$.

Before discussing the construction of $\sigma_{G}$, we review a Clifford type theorem for coherent systems established by Newstead-Lange [LN08]. For a smooth projective curve $C$ and $\alpha \in \mathbb{R}_{>0}$, recall that the $\alpha$-stability on $\operatorname{Syst}(C)$ is defined by the slope function

$$
\left(\mathcal{O}_{C}^{\oplus R} \rightarrow F\right) \mapsto \frac{d(F)+\alpha \cdot R}{r(F)} .
$$

The slope function is set to be $\infty$ if $r(F)=0$.

TheOrem 5.16. ([LN08, Theorem 2.1, Remark 2.3]) Let $C$ be a smooth projective curve of genus $g(C) \geqslant 2$, and let $\alpha \in \mathbb{R}_{>0}$. Then for any $\alpha$-stable coherent system $\left(\mathcal{O}_{C}^{\oplus R} \rightarrow F\right)$ with $0 \leqslant d(F)<2 g(C) \cdot r(F)$, we have

$$
R \leqslant \frac{d(F)}{2}+r(F)
$$

Moreover, if $C$ is non-hyperelliptic, then equality holds in (111) only if $\left(\mathcal{O}_{X}^{\oplus R} \rightarrow F\right)$ is isomorphic to either

$$
H^{0}\left(\mathcal{O}_{C}\right) \otimes \mathcal{O}_{C} \rightarrow \mathcal{O}_{C} \quad \text { or } H^{0}\left(\omega_{C}\right) \otimes \mathcal{O}_{C} \rightarrow \omega_{C}
$$

Using this result, we have the following lemma, which plays a crucial role in constructing a Gepner type stability condition on $\operatorname{HMF}^{\mathrm{gr}}(W)$.

Lemma 5.17. Let $\left(\mathcal{O}_{X}^{\oplus R} \stackrel{s}{\rightarrow} F\right)$ be a $\mu$-stable object in $\mathcal{A}_{W}$ such that $R=2 r(F)>0$. Then we have

$$
d(F)>R\left(1-\cos \frac{2 \pi}{d}\right) .
$$

Proof. By the $\mu$-stability, the morphism $s$ is surjective (cf. Remark 5.15) hence $d(F) \geqslant 0$. Also, the right-hand side of (113) is $R$ if $d=4$ and $R / 2$ if $d=6$. In both cases, it is smaller than $2 g(X) \cdot r(F)=g(X) \cdot R$, so we may assume $d(F)<2 g(X) \cdot r(F)$. Comparing the $\mu$-stability in (108) and the $\alpha$-stability in (110), we see that the $\mu$-stable object $\left(\mathcal{O}_{X}^{\oplus R} \rightarrow F\right)$ is $\alpha$-stable for $\alpha \gg 0$, since the set of quotient sheaves of $F$ with degrees bounded from above is bounded. Therefore, applying Theorem 5.16, we obtain the inequality $d(F) \geqslant R$. It remains to check that the equality $d(F)=R$ does not hold if $d=4$. In this case, $X$ is a quartic curve, so it is not hyperelliptic. Also, $H^{0}\left(\mathcal{O}_{C}\right)$ is one-dimensional, $H^{0}\left(\omega_{C}\right)$ is three dimensional, hence the objects in (112) do not satisfy our assumption $R=2 r(F)$. Therefore the case $d(F)=R$ is excluded. 


\section{YuKINOBU TODA}

As we discussed in Subsection 4.3, the slope function $\mu$ defines a torsion pair (68) on $\mathcal{A}_{W}$, and the associated tilting $\mathcal{A}_{G}$ is given by (69). We have the following result.

Proposition 5.18. Suppose $n=3$ and $\varepsilon=-1$. Then the triple

$$
\left(Z_{G}, \mathcal{A}_{G}, \theta=\theta_{W}\right)
$$

determines a Gepner type stability condition $\sigma_{G}$ on $\operatorname{HMF}^{\mathrm{gr}}(W)$ with respect to $(\tau, 2 / d)$.

Proof. Obviously, the triple (114) determines a stability condition if and only if the triple

$$
\left(Z_{G}^{\dagger}=Z_{G} / C_{W}, \mathcal{A}_{G}, \theta=0\right)
$$

determines a stability condition. By the construction of $\mathcal{A}_{G}$, any non-zero object $E \in \mathcal{A}_{G}$ satisfies $\operatorname{Im} Z_{G}^{\dagger}(E) \geqslant 0$. Moreover, Lemma 5.13 and Lemma 5.17 imply that if $\operatorname{Im} Z_{G}^{\dagger}(E)=0$, then $\operatorname{Re} Z_{G}^{\dagger}(E)<0$. Therefore the triple (115) satisfies condition (71). Also, by Lemma 5.13, the image of $Z_{G}^{\dagger}$ is discrete, which enables us to apply the same argument of [Bri08, Proposition 7.1] to prove the Harder-Narasimhan property of the triple (115). Therefore the triple (115), and therefore (114), determines a stability condition. Let

$$
\sigma_{G}=\left(Z_{G},\left\{\mathcal{P}_{G}(\phi)\right\}_{\phi \in \mathbb{R}}\right)
$$

be the stability condition on $\operatorname{HMF}^{\mathrm{gr}}(W)$ determined by the triple (114). We need to show that $\sigma_{G}$ is of Gepner type with respect to $(\tau, 2 / d)$. Note that, in our situation, the triple (114) satisfies the inequality in (83). Therefore, by Lemma 4.12, it is enough to check the $\sigma_{G}$-stability of $\tau \Psi\left(\mathcal{O}_{x}\right)$, $\mathbb{C}(1)$ and $\tau^{2} \Psi\left(\mathcal{O}_{x}\right)$. The stability of these objects is proved in Lemma 5.19, Lemma 5.21 and Lemma 5.23 below.

Below we check the $\sigma_{G}$-stability of the objects $\tau \Psi\left(\mathcal{O}_{x}\right), \mathbb{C}(1)$ and $\tau^{2} \Psi\left(\mathcal{O}_{x}\right)$. Let $\sigma_{G}^{\dagger}$ be the stability condition on $\mathrm{HMF}^{\text {gr }}(W)$ determined by the triple (115). It differs from $\sigma_{G}$ by an action of $\mathbb{C}$, so it is enough to check the $\sigma_{G}^{\dagger}$-stability of these objects.

Lemma 5.19. For any $x \in X$, the object $\tau \Psi\left(\mathcal{O}_{x}\right)$ is $\sigma_{G}^{\dagger}$-stable.

Proof. By Lemma 3.10, the object $\tau \Psi\left(\mathcal{O}_{x}\right)$ is an object in $\mathcal{A}_{W}$, given by the coherent system $\left(\mathcal{O}_{X} \rightarrow \mathcal{O}_{x}\right)$. It is $\mu$-stable with $\mu(*)>0$, hence $\tau \Psi\left(\mathcal{O}_{x}\right)[-1] \in \mathcal{A}_{G}$. On the other hand, we have

$$
\operatorname{Im} Z_{G}^{\dagger}\left(\mathcal{A}_{G}\right) \subset\left\{\frac{\sqrt{\widehat{H}^{2} d}}{4} \times \mathbb{Z}_{\geqslant 0}\right\} .
$$

The imaginary part of $Z_{G}^{\dagger}\left(\tau \Psi\left(\mathcal{O}_{x}\right)[-1]\right)$ is $\sqrt{\widehat{H}^{2} d} / 4$, which is the smallest positive number in the right-hand side of (116). By Lemma 4.14, it is enough to check that there is no non-zero morphism from any object $P \in \mathcal{A}_{G}$ with $\operatorname{Im} Z_{G}^{\dagger}(P)=0$ to $\Psi\left(\mathcal{O}_{x}\right)[-1]$. Since $\tau \Psi\left(\mathcal{O}_{x}\right)[-1] \in \mathcal{A}_{W}[-1]$, and $P \in \mathcal{A}_{W}$ by Sublemma 5.20 below, there is no non-zero morphism from $P$ to $\tau \Psi\left(\mathcal{O}_{x}\right)[-1]$.

We have used the following sublemma. The proof is obvious from the construction of $\mathcal{A}_{G}$, and we omit it.

Sublemma 5.20. A non-zero object $P \in \mathcal{A}_{G}$ satisfies $\operatorname{Im} Z_{G}^{\dagger}(P)=0$ if and only if $P \in \mathcal{A}_{W}$, and it is given by iterated extensions of $\mu$-stable coherent systems $\left(\mathcal{O}_{X}^{\oplus R} \rightarrow F\right)$ with $\mu\left(\mathcal{O}_{X}^{\oplus R} \rightarrow F\right)=0$, and coherent systems of the form $\left(0 \rightarrow \mathcal{O}_{y}\right)$ for $y \in X$.

Next we check the stability of $\mathbb{C}(1)$. 


\section{GePNER TyPE STABILITy CONDITIONS}

Lemma 5.21. The object $\mathbb{C}(1)$ is $\sigma_{G}^{\dagger}$-stable.

Proof. By Corollary 3.23, we have the isomorphism (again we leave out $\Theta$ )

$$
\mathbb{C}(1)[-1] \cong\left(\mathcal{O}_{X} \otimes R_{1} \stackrel{s}{\rightarrow} \mathcal{O}_{X}(1)\right)
$$

such that $s$ is the natural evaluation map. In particular, $H^{0}(s)$ is an isomorphism, and $s$ is surjective since $\mathcal{O}_{X}(1)$ is globally generated. Then we apply Sublemma 5.22 below to show that $\mathbb{C}(1)[-1]$ is $\mu$-stable. The slope $\mu(\mathbb{C}(1)[-1])$ equals $1 / 6$ if $d=4$ and 0 if $d=6$. In the former case, $\mathbb{C}(1)[-2] \in \mathcal{A}_{G}$, and the imaginary part of $Z_{G}^{\dagger}(\mathbb{C}(1)[-2])$ is 1 , which is the smallest positive value of the right-hand side of (116). Hence the $\sigma_{G}^{\dagger}$-stability of $\mathbb{C}(1)[-2]$ follows from the same argument as Lemma 5.19. In the latter case, $\mathbb{C}(1)[-1] \in \mathcal{A}_{G}$ and the imaginary part of $Z_{G}^{\dagger}(\mathbb{C}(1)[-1])$ is 0 . Hence the $\sigma_{G}^{\dagger}$-stability of $\mathbb{C}(1)[-1]$ follows from its $\mu$-stability and Sublemma 5.20.

Sublemma 5.22. An object $\left(\mathcal{O}_{X}^{\oplus R} \stackrel{s}{\rightarrow} \mathcal{L}\right) \in \mathcal{A}_{W}$ with $R>0$ and $\mathcal{L} \in \operatorname{Pic}(X)$ is $\mu$-stable if $s$ is surjective and $H^{0}(s)$ is injective.

Proof. Suppose that $s$ is surjective and $H^{0}(s)$ is injective. Let

$$
0 \rightarrow\left(\mathcal{O}_{X}^{\oplus R_{1}} \rightarrow \mathcal{L}_{1}\right) \rightarrow\left(\mathcal{O}_{X}^{\oplus R} \rightarrow \mathcal{L}\right) \rightarrow\left(\mathcal{O}_{X}^{\oplus R_{2}} \rightarrow \mathcal{L}_{2}\right) \rightarrow 0
$$

be an exact sequence of coherent systems. It is enough to show the inequality

$$
\mu\left(\mathcal{O}_{X}^{\oplus R} \rightarrow \mathcal{L}\right)<\mu\left(\mathcal{O}_{X}^{\oplus R_{2}} \rightarrow \mathcal{L}_{2}\right) .
$$

By our assumption, $\mathcal{L}_{1} \neq 0$ and $R_{2} \neq 0$, hence $\mathcal{L}_{2}$ is a zero-dimensional sheaf. Therefore the right-hand side of (117) equals to $1 / 2$, while the left-hand side of (117) is less than $1 / 2$. Hence (117) holds.

It remains to prove the following lemma.

Lemma 5.23. The object $\tau^{2} \Psi\left(\mathcal{O}_{x}\right)$ is an object in $\mathcal{P}_{G}\left(\theta_{W}+1+4 / d\right)$.

Proof. Applying $\tau$ to the exact sequence (32), we obtain the distinguished triangle

$$
\tau \Psi\left(\mathcal{O}_{x}\right) \rightarrow \tau^{2} \Psi\left(\mathcal{O}_{x}\right) \rightarrow \mathbb{C}(1)
$$

in $\operatorname{HMF}^{\mathrm{gr}}(W)$. Combining this with Lemma 3.10 and Corollary 3.23, we see that the object $\tau^{2} \Psi\left(\mathcal{O}_{x}\right)$ is obtained as a cone of the morphism of coherent systems:

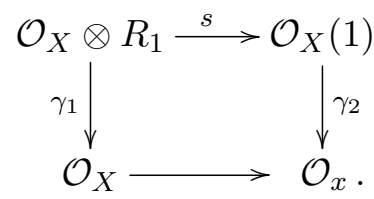

Here $s$ is the evaluation map and $\left(\gamma_{1}, \gamma_{2}\right)$ is the morphism of coherent systems. Since $\left(\gamma_{1}, \gamma_{2}\right)$ is non-zero, $\gamma_{1}$ and $\gamma_{2}$ are both non-zero, hence surjective. Therefore the object $\tau^{2} \Psi\left(\mathcal{O}_{x}\right)[-1]$ is given by the coherent system

$$
\left(\mathcal{O}_{X} \otimes R_{1, x} \stackrel{s_{x}}{\rightarrow} \mathcal{O}_{X}(1) \otimes I_{x}\right) .
$$

Here $R_{1, x}$ is the subspace of $R_{1}$ which vanishes at $x$, and $I_{x}$ is the ideal sheaf which defines $x$.

First, suppose $d=4$. In this case, $R_{1, x}$ is two-dimensional, and $s_{x}$ is surjective since any of two lines in $\mathbb{P}^{2}$ determined by two generic elements in $R_{1, x}$ intersect only at $x$ transversally Also, $H^{0}\left(s_{x}\right)$ is injective since $H^{0}(s)$ is an isomorphism. Therefore the coherent system (118) 


\section{YukINOBU TODA}

is $\mu$-stable by Sublemma 5.22. Since $\mu=0$ for the coherent system $(118), \tau^{2} \Psi\left(\mathcal{O}_{x}\right)[-1]$ is an element of $\mathcal{A}_{G}$ and is $\sigma_{G}$-stable. In particular, $\tau^{2} \Psi\left(\mathcal{O}_{x}\right)$ is an object in $\mathcal{P}_{G}\left(\theta_{W}+2\right)$.

Next, suppose $d=6$. In this case, $R_{1, x}$ is one-dimensional, and $s_{x}$ is not surjective at $x$. In particular, the coherent system (118) is not $\mu$-semi-stable. However, we can show the $Z_{G^{-}}^{\dagger}$ stability, hence the $\sigma_{G}^{\dagger}$-stability, of (118) in the following way: the sheaf $\mathcal{O}_{X}(1) \otimes I_{x}$ is isomorphic to $\mathcal{O}_{X}\left(x^{\prime}\right)$ for another point $x^{\prime} \in X$, and (118) is isomorphic to the coherent system

$$
\left(\mathcal{O}_{X} \stackrel{s^{\prime}}{\rightarrow} \mathcal{O}_{X}\left(x^{\prime}\right)\right)
$$

where $s^{\prime}$ is a natural inclusion. There is an exact sequence of coherent systems

$$
0 \rightarrow\left(\mathcal{O}_{X} \stackrel{\text { id }}{\rightarrow} \mathcal{O}_{X}\right) \rightarrow\left(\mathcal{O}_{X} \rightarrow \mathcal{O}_{X}\left(x^{\prime}\right)\right) \rightarrow\left(0 \rightarrow \mathcal{O}_{x^{\prime}}\right) \rightarrow 0
$$

Both of the objects $\left(\mathcal{O}_{X} \stackrel{\text { id }}{\rightarrow} \mathcal{O}_{X}\right)$ and $\left(0 \rightarrow \mathcal{O}_{x^{\prime}}\right)$ are $\mu$-stable with negative slopes, hence the object (118) is an object in $\mathcal{A}_{G}$. Also the imaginary part of $Z_{G}^{\dagger}\left(\mathcal{O}_{X} \rightarrow \mathcal{O}_{X}\left(x^{\prime}\right)\right)$ is $\sqrt{3} / 2$, which is the smallest positive value of the right-hand side of (116). Therefore by Lemma 4.14, it is enough to check that there is no non-zero morphism from $P \in \mathcal{A}_{G}$ with $\operatorname{Im} Z_{G}^{\dagger}(P)=0$ to the object $\left(\mathcal{O}_{X} \rightarrow \mathcal{O}_{X}\left(x^{\prime}\right)\right)$. This follows from Sublemma 5.20 , since $\left(\mathcal{O}_{X} \stackrel{\text { id }}{\rightarrow} \mathcal{O}_{X}\right)$ and $\left(0 \rightarrow \mathcal{O}_{x^{\prime}}\right)$ are both $\mu$-stable with negative slopes, and the exact sequence (119) does not split.

5.5 The case $n=2, \varepsilon=-2$

Finally, in this subsection, we study the case $(n, \varepsilon)=(2,-2)$. In this case, $X$ is a finite number of smooth points, represented by points $p^{(j)}=\left(p_{1}^{(j)}, p_{2}^{(j)}\right) \in \mathbb{C}^{2}$ for $1 \leqslant j \leqslant \sharp X$. The heart $\mathcal{A}_{W}$ is given by

$$
\mathcal{A}_{W}=\left\langle\mathbb{C}(1), \mathbb{C}(0), \Psi\left(\mathcal{O}_{x}\right): x \in X\right\rangle_{\mathrm{ex}} .
$$

By Lemma 3.17 and Lemma 3.18 , the heart $\mathcal{A}_{W}$ is equivalent to the abelian category of representations of a certain quiver $\mathcal{Q}$ with relations. As in the previous subsection, we have the following possibilities:

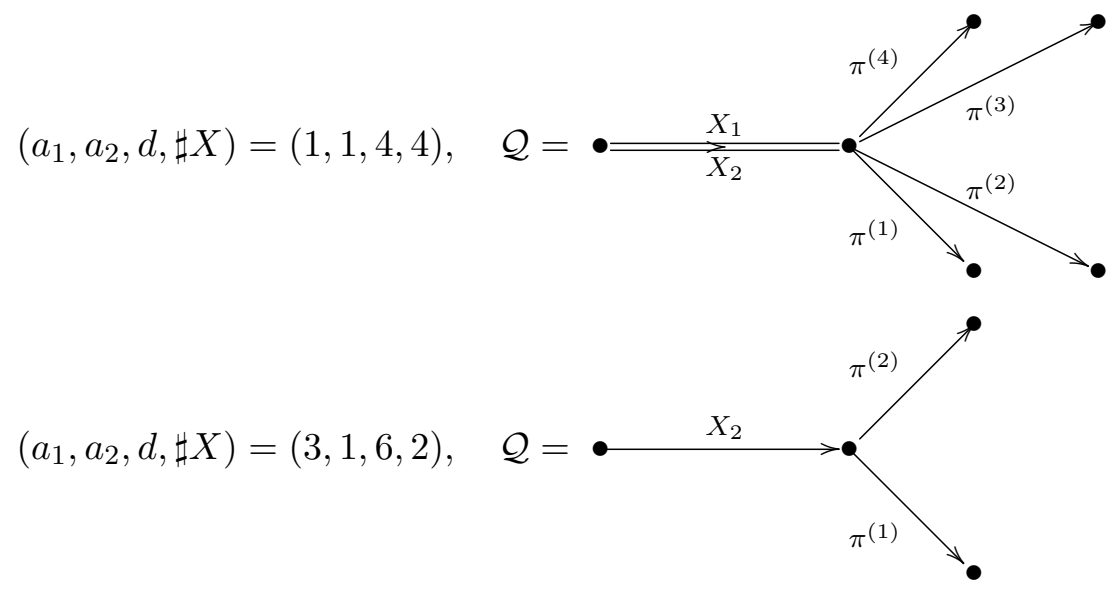

Also, by Corollary 3.19, in the $d=4$ case, we have the following relations:

$$
p_{2}^{(j)} \pi^{(j)} X_{1}=p_{1}^{(j)} \pi^{(j)} X_{2}, \quad 1 \leqslant j \leqslant 4 .
$$

There is no relation in the $d=6$ case. By (66) and the classification above, the central charge $Z_{G}$ is given as follows. 


\section{GePner TyPE STABILITy CONDITIONS}

Lemma 5.24. If we write the $K$-theory class of $E \in \mathcal{A}_{W}$ as

$$
[E]=v_{1}[\mathbb{C}(1)]+v_{0}[\mathbb{C}(0)]+\sum_{j=1}^{\sharp X} w_{j}\left[\Psi\left(\mathcal{O}_{p^{(j)}}\right)\right]
$$

for $v_{j}, w_{j} \in \mathbb{Z}_{\geqslant 0}$, then we have

$$
\begin{aligned}
Z_{G}(E)=C_{W} & \left\{-w+\left(1-\cos \frac{2 \pi}{d}\right) v_{0}+v_{1}\right. \\
+ & \left.\left\{-\sin \frac{2 \pi}{d} v_{0}+\left(\sin \frac{2 \pi}{d}-\sin \frac{4 \pi}{d}\right) v_{1}\right\} \sqrt{-1}\right\},
\end{aligned}
$$

where $w:=\sum_{j=1}^{\sharp X} w_{j}$.

By setting $Z_{G}^{\dagger}:=Z_{G} / C_{W}$, we define the slope function $\mu$ on $\mathcal{A}_{W}$ by

$$
\begin{aligned}
\mu(E) & :=\frac{\operatorname{Re} Z_{G}^{\dagger}(E)}{w} \\
& =\frac{1}{w}\left(v_{1}+\left(1-\cos \frac{2 \pi}{d}\right) v_{0}\right)-1 .
\end{aligned}
$$

We set $\mu(E)=\infty$ if $w=0$. This slope function defines the $\mu$-stability on $\mathcal{A}_{W}$. Since $\mathcal{A}_{W}$ is noetherian and artinian, any object in $\mathcal{A}_{W}$ admits a $\mu$-Harder-Narasimhan filtration (cf. [Tod10a, Proposition 2.12]). We have the following lemma.

Lemma 5.25. For any non-zero $\mu$-stable object $E \in \mathcal{A}_{W}$ with $\mu(E)=0$, we have $\operatorname{Im} Z_{G}^{\dagger}(E)<0$.

Proof. In the case $d=6$, we have $\operatorname{Im} Z_{G}^{\dagger}(E)=-\sqrt{3} v_{0} / 2$, which is non-positive. If $v_{0}=0$, the condition $\mu(E)=0$ implies $v_{1}=w \neq 0$. Therefore $E$ decomposes into direct sums, which contradicts the $\mu$-stability of $E$.

In the case $d=4$, the claim is equivalent to the claim that if $E$ is $\mu$-stable with $v_{0}+v_{1}=w \neq 0$, then $v_{1}<v_{0}$. Let us represent $E$ as a representation of $\mathcal{Q}$ :

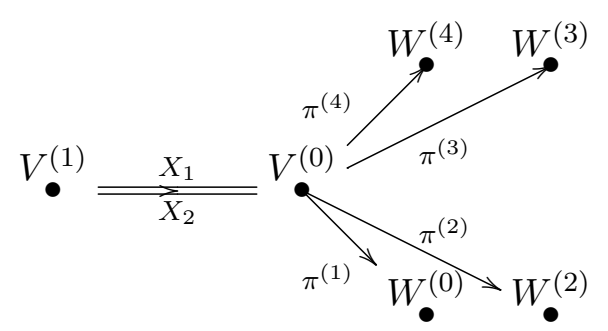

where the $V^{(i)}$ are $v_{i}$-dimensional and the $W^{(i)}$ are $w_{i}$-dimensional. Suppose that $v_{1} \geqslant v_{0}$. By the relation (120), for $1 \leqslant i \leqslant 4$, we have the linear maps

$$
p_{1}^{(i)} X_{2}-p_{2}^{(i)} X_{1}: V^{(1)} \rightarrow \operatorname{Ker}\left(\pi^{(i)}\right) .
$$

Because of the condition $w \neq 0$, there is an $1 \leqslant i \leqslant 4$ such that $w^{(i)} \neq 0$. Also, the $\mu$-stability of $E$ implies that $\pi^{(i)}$ is surjective. Therefore the assumption $v_{1} \geqslant v_{0} \operatorname{implies} \operatorname{dim} V^{(1)}>\operatorname{dim} \operatorname{Ker}\left(\pi^{(i)}\right)$, and there is a non-zero element $v \in V^{(1)}$ which is mapped to zero by (123). Let us consider the sub-quiver representation of (122) generated by $v$. By taking relation (120) into consideration, the corresponding subobject $F \subset E$ in $\mathcal{A}_{W}$ has K-theory class

$$
[\mathbb{C}(1)] \text { or }[\mathbb{C}(1)]+[\mathbb{C}(0)] \text { or }[\mathbb{C}(1)]+[\mathbb{C}(0)]+\left[\Psi\left(\mathcal{O}_{p^{(i)}}\right)\right]
$$




\section{YUKINOBU TODA}

Hence we have $\mu(F)>0$, which contradicts to the $\mu$-stability of $E$ with $\mu(E)=0$.

As before, the slope function $\mu$ defines a torsion pair (68) on $\mathcal{A}_{W}$, and the associated tilting $\mathcal{A}_{G}$ given by (69). We have the following result.

Proposition 5.26. Suppose $n=2$ and $\varepsilon=-2$. Then the triple

$$
\left(Z_{G}, \mathcal{A}_{G}, \theta=\theta_{W}+\frac{1}{2}\right)
$$

determines a Gepner type stability condition $\sigma_{G}$ on $\operatorname{HMF}^{\mathrm{gr}}(W)$ with respect to $(\tau, 2 / d)$.

Proof. The triple (124) determines a stability condition if and only if the triple

$$
\left(Z_{G}^{\dagger}=Z_{G} / C_{W}, \mathcal{A}_{G}, \theta=\frac{1}{2}\right)
$$

determines a stability condition. By Lemma 5.24, Lemma 5.25 and the construction of $\mathcal{A}_{G}$, the triple (125) satisfies condition (71). Also, since the image of $Z_{G}^{\dagger}$ is discrete, the same argument as that used for [Bri08, Proposition 7.1] shows the Harder-Narashiman property of (125). Therefore the triples (124), (125) determine stability conditions $\sigma_{G}, \sigma_{G}^{\dagger}$ respectively. Since they only differ by a $\mathbb{C}$-action, by Lemma 4.10 , it is enough to check the $\sigma_{G}^{\dagger}$-stability of $\tau \Psi\left(\mathcal{O}_{x}\right), \mathbb{C}(1)$ and $\mathbb{C}(2)$. These are checked in Lemma 5.27, Lemma 5.28 and Lemma 5.29 below.

Lemma 5.27. For any $x \in X$, the object $\tau \Psi\left(\mathcal{O}_{x}\right)$ is $\sigma_{G}^{\dagger}$-stable.

Proof. By Lemma 3.10, we have $\tau \Psi\left(\mathcal{O}_{x}\right) \in \mathcal{A}_{W}$. By the exact sequence (32), the object $\tau \Psi\left(\mathcal{O}_{x}\right)$ is $\mu$-stable with non-positive slope. Therefore $\tau \Psi\left(\mathcal{O}_{x}\right)$ is an object in $\mathcal{A}_{G}$. In the $d=4$ case, we have $Z_{G}^{\dagger}\left(\tau \Psi\left(\mathcal{O}_{x}\right)\right)=-\sqrt{-1}$. Since the image of $Z_{G}^{\dagger}$ is $\mathbb{Z}+\mathbb{Z} \sqrt{-1}$, this immediately implies that $\tau \Psi\left(\mathcal{O}_{x}\right)$ is $\sigma_{G}^{\dagger}$-stable. In the $d=6$ case, note that $\operatorname{Re} Z_{G}^{\dagger}(*)$ on $\mathcal{A}_{G}$ is contained in $\mathbb{Z}_{\leqslant 0} \times 1 / 2$. Since $\operatorname{Re} Z_{G}^{\dagger}\left(\tau \Psi\left(\mathcal{O}_{x}\right)\right)=-1 / 2$, by Lemma 4.14, it is enough to check that $\operatorname{Hom}\left(P, \tau \Psi\left(\mathcal{O}_{x}\right)\right)=0$ for any $P \in \mathcal{A}_{G}$ with $\operatorname{Re} Z_{G}^{\dagger}(P)=0$. By our construction of $\mathcal{A}_{G}$, we have $P \in \mathcal{A}_{W}$, and $P$ is $\mu$-semi-stable with $\mu(P)=0$. Since $\tau \Psi\left(\mathcal{O}_{x}\right)$ is $\mu$-stable with negative slope, we have $\operatorname{Hom}\left(P, \tau \Psi\left(\mathcal{O}_{x}\right)\right)=0$.

Lemma 5.28. The object $\mathbb{C}(1)$ is $\sigma_{G}^{\dagger}$-stable.

Proof. Since $\mathbb{C}(1)$ is $\mu$-stable with $\mu(\mathbb{C}(1))=\infty$, we have $\mathbb{C}(1)[-1] \in \mathcal{A}_{G}$. In the $d=4$ case, note that $\operatorname{Re} Z_{G}^{\dagger}(*)$ is contained in $\mathbb{Z}_{\leqslant 0}$. Since $\operatorname{Re} Z_{G}^{\dagger}(\mathbb{C}(1)[-1])=-1$, by Lemma 4.14 , it is enough to check that $\operatorname{Hom}(P, \mathbb{C}(1)[-1])=0$ for any $P \in \mathcal{A}_{G}$ with $\operatorname{Re} Z_{G}^{\dagger}(P)=0$, which follows since $P$ is an object in $\mathcal{A}_{W}$. In the $d=6$ case, let $E$ be a non-trivial quotient of $\mathbb{C}(1)[-1]$ in $\mathcal{A}_{G}$. Since $Z_{G}^{\dagger}(\mathbb{C}(1)[-1])=-1$, it is enough to check that $\operatorname{Im} Z_{G}^{\dagger}(E)<0$. To check this, note that we have $E \in \mathcal{A}_{W}$ since $\mathbb{C}(1)$ is a simple object in $\mathcal{A}_{W}$. Let us write the K-theory class of $E$ as in (121). Since $\operatorname{Im} Z_{G}^{\dagger}(E)=-\sqrt{3} v_{0} / 2$ is non-positive, it is enough to exclude the case $v_{0}=0$. If $v_{0}=0$, then $E$ is a direct sum of objects $\mathbb{C}(1)$ and $\Psi\left(\mathcal{O}_{x}\right)$ for $x \in X$. Then there is a non-zero morphism from $\mathbb{C}(1)[-1]$ to $\Psi\left(\mathcal{O}_{x}\right)$, which contradicts to Lemma 3.17 .

Lemma 5.29. The object $\mathbb{C}(2)$ is $\sigma_{G}^{\dagger}$-stable.

Proof. We first discuss the case $d=4$. In this case, by Corollary 3.20 , the object $\mathbb{C}(2)[-1]$ is an 


\section{GePNER TyPE STABILITy CONDITIONS}

object in $\mathcal{A}_{W}$ represented by the following quiver representation:

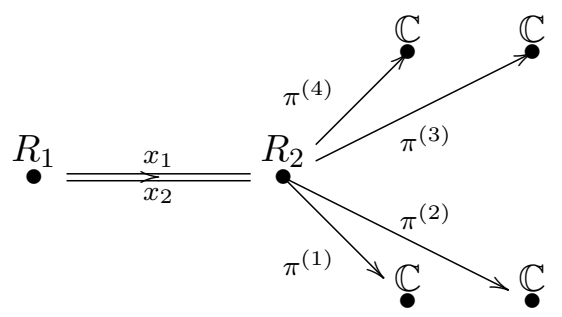

Here $R_{1}$ is two-dimensional and $R_{2}$ is three-dimensional. We first show that $\mathbb{C}(2)[-1]$ is $\mu$-stable. Let $E$ be a subobject of $\mathbb{C}(2)[-1]$ in $\mathcal{A}_{W}$ given by a representation (122), with $v^{(j)}:=\operatorname{dim} V^{(j)}$ and $w:=\sum_{j=1}^{4} \operatorname{dim} W^{(j)}$. Let $I$ be the subset of $i \in\{1,2,3,4\}$ such that $W^{(i)}=0$. Similarly to the proof of Lemma 5.5, we have the inequality $v^{(0)} \leqslant 3-\sharp I$. Also, we may assume $v^{(1)} \leqslant 1$, since otherwise $F$ coincides with $\mathbb{C}(2)[-1]$. Therefore we have the inequality

$$
v^{(0)}+v^{(1)} \leqslant 4-|I|=w,
$$

which implies that $\mu(E) \leqslant 0<\mu(\mathbb{C}(2)[-1])=1 / 4$. Therefore $\mathbb{C}(2)[-1]$ is $\mu$-stable with positive slope, hence we have $\mathbb{C}(2)[-2] \in \mathcal{A}_{G}$. Since $Z_{G}^{\dagger}(\mathbb{C}(2)[-2])=-1+\sqrt{-1}$ and the image of $Z_{G}^{\dagger}$ is $\mathbb{Z}+$ $\mathbb{Z} \sqrt{-1}$, by Lemma 4.14 , the $\sigma_{G}^{\dagger}$-stability of $\mathbb{C}(2)[-2]$ follows if we check that $\operatorname{Hom}(P, \mathbb{C}(2)[-2])=$ 0 for any $P \in \mathcal{A}_{G}$ with $\operatorname{Re} Z_{G}^{\dagger}(P)=0$. This follows since $P \in \mathcal{A}_{W}$ and $\mathbb{C}(2)[-2] \in \mathcal{A}_{W}[-1]$.

Next we discuss the case $d=6$. By Lemma 3.11, the object $\mathbb{C}(2)[-1] \in \mathcal{A}_{W}$ is represented by the following quiver representation

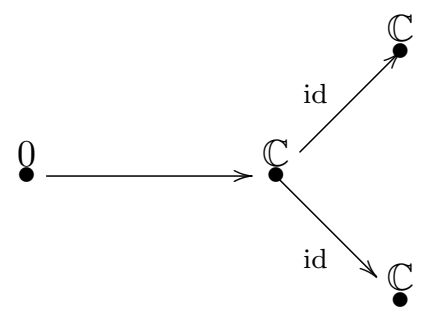

This object is obviously $\mu$-stable. Since $\mu(\mathbb{C}(2)[-1])=-3 / 4$, it follows that $\mathbb{C}(2)[-1] \in \mathcal{A}_{G}$. Also we have $Z_{G}^{\dagger}(\mathbb{C}(2)[-1])=-(1 / 2+3 \sqrt{-3} / 2)$, and $\operatorname{Re} Z_{G}^{\dagger}(*)$ is contained in $\mathbb{Z}_{\leqslant 0} \times 1 / 2$ on $\mathcal{A}_{G}$. Applying Lemma 4.14, the $\sigma_{G}^{\dagger}$-stability of $\mathbb{C}(2)[-1]$ follows if we check that $\operatorname{Hom}(P, \mathbb{C}(2)[-1])=0$ for any $P \in \mathcal{A}_{G}$ with $\operatorname{Re} Z_{G}^{\dagger}(P)=0$. Since such $P$ is a $\mu$-semi-stable object in $\mathcal{A}_{W}$ with $\mu(P)=0$, and $\mathbb{C}(2)[-1]$ is $\mu$-stable with negative slope, it follows that we have $\operatorname{Hom}(P, \mathbb{C}(2)[-1])=0$.

\section{ACKNOWLEDGEMENTS}

The author would like to thank Kentaro Hori, Kyoji Saito and Atsushi Takahashi for valuable discussions.

\section{REFERENCES}

BFK12 M. Ballard, D. Favero and L. Katzarkov, Orlov spectra: bounds and gaps, Invent. Math. 189 (2012), 359-430. http://dx.doi.org/10.1007/s00222-011-0367-y

BM11 A. Bayer and E. Macri, The space of stability conditions on the local projective plane, Duke Math. J. 160 (2011), 263-322. http://dx.doi.org/10.1215/00127094-1444249 


\section{YUKINOBU TODA}

BMT14 A. Bayer, E. Macri and Y. Toda, Bridgeland stability conditions on threefolds I: BogomolovGieseker type inequalities, J. Algebraic Geom. 23 (2014), 117-163. http://dx.doi.org/10. 1090/S1056-3911-2013-00617-7

Bri07 T. Bridgeland, Stability conditions on triangulated categories, Ann. of Math. 166 (2007), 317345. http://dx.doi.org/10.4007/annals.2007.166.317

Bri08_, Stability conditions on K3 surfaces, Duke Math. J. 141 (2008), 241-291. http://dx. doi.org/10.1215/S0012-7094-08-14122-5

Bri11_ Hall algebras and curve-counting invariants, J. Amer. Math. Soc. 24 (2011), 969-998. http://dx.doi.org/10.1090/S0894-0347-2011-00701-7

Căl05 A. Căldăraru, The Mukai pairing, II: The Hochschild-Kostant-Rosenberg isomorphism, Adv. Math. 194 (2005), 34-66. http://dx.doi:10.1016/j.aim.2004.05.012

CP10 J. Collins and A. Polishchuk, Gluing stability conditions, Adv. Theor. Math. Phys. 14 (2010), 563-607. http://dx.doi.org/10.4310/ATMP.2010.v14.n2.a6

CR10 A. Chiodo and Y. Ruan, Landau-Ginzburg/Calabi-Yau correspondence for quintic three-folds via symplectic transformations, Invent. Math. 182 (2010), 117-165. http://dx.doi.org/10. 1007/s00222-010-0260-0

DM07 F. Denef and G. Moore, Split states, Entropy Enigmas, Holes and Halos, arXiv:hep-th/0702146.

Dyc11 T. Dyckerhoff, Compact generators in categories of matrix factorizations, Duke Math. J. 159 (2011), 223-274. http://dx.doi.org/10.1215/00127094-1415869

FJR07 H. Fan, T. J. Jarvis, and Y. Ruan, The Witten equation and its virtual fundamental cycle, arXiv:0712.4025.

HRS96 D. Happel, I. Reiten and S. O. Smalø, Tilting in abelian categories and quasitilted algebras, Mem. Amer. Math. Soc. 120 (1996), no. 575.

JS12 D. Joyce and Y. Song, A theory of generalized Donaldson-Thomas invariants, Mem. Amer. Math. Soc. 217 (2012), no. 1020. http://dx.doi.org/10.1090/ S0065-9266-2011-00630-1

Knö87 H. Knörrer, Cohen-Macauley modules on hypersurface singularities, Invent. Math. 88 (1987), 153-164. http://dx.doi.org/10.1007/BF01405095

KS08 M. Kontsevich and Y. Soibelman, Stability structures, motivic Donaldson-Thomas invariants and cluster transformations, arXiv:0811.2435.

KST07 H. Kajiura, K. Saito and A. Takahashi, Matrix factorizations and representations of quivers II. Type ADE case, Adv. Math. 211 (2007), 327-362. http://dx.doi.org/10.1016/j.aim. 2006. 08.005

KST09 , Triangulated categories of matrix factorizations for regular systems of weights $\varepsilon=-1$, Adv. Math. 220 (2009), 1602-1654. http://dx.doi.org/10.1016/j.aim.2008.11.001

LN08 H. Lange and P. E. Newstead, Clifford's theorem for coherent systems, Arch. Math. 90 (2008), 209-216. http://dx.doi.org/10.1007/s00013-007-2534-3

Orl09 D. Orlov, Derived categories of coherent sheaves and triangulated categories of singularities, in Algebra, arithmetic, and geometry: in honor of Yu. I. Manin, 503-531, Progr. Math. 270, Birkhäuser Boston, Inc., Boston, MA, 2009. http://dx.doi.org/10.1007/ 978-0-8176-4747-6_16

OSV04 H. Ooguri, A. Strominger and C. Vafa, Black hole attractors and the topological string, Phys. Rev. D 70 (2004). http://dx.doi.org/10.1103/PhysRevD.70.106007

PV11 A. Polishchuk and A. Vaintrob, Matrix factorizations and Cohomological Field Theories, arXiv:1105.2903.

PV12 , Chern characters and Hirzebruch-Riemann-Roch formula for matrix factorizations, Duke Math. J. 161 (2012), 1863-1926. http://dx.doi.org/10.1215/00127094-1645540

Sai87 K. Saito, Regular system of weights and associated singularities, in Complex analytic singularities, 479-526, Adv. Stud. Pure Math. 8, North-Holland, Amsterdam, 1987. 


\section{GEPNER TYPE STABILITY CONDITIONS}

ST01 P. Seidel and R. P. Thomas, Braid group actions on derived categories of coherent sheaves, Duke Math. J. 108 (2001), 37-107. http://dx.doi.org/10.1215/S0012-7094-01-10812-0

Tak05 A. Takahashi, Matrix factorizations and representations of quivers I, arXiv:0506347.

Tho00 R. P. Thomas, A holomorphic Casson invariant for Calabi-Yau 3-folds and bundles on K3fibrations, J. Differential. Geom. 54 (2000), 367-438.

Tod08 , Moduli stacks and invariants of semistable objects on K3 surfaces, Adv. Math. 217 (2008), 2736-2781. http://dx.doi.org/10.1016/j.aim.2007.11.010

Tod09_, Limit stable objects on Calabi-Yau 3-folds, Duke Math. J. 149 (2009), 157-208. http: //dx.doi.org/10.1215/00127094-2009-038

Tod10a , Curve counting theories via stable objects I: DT/PT correspondence, J. Amer. Math. Soc. 23 (2010), 1119-1157. http://dx.doi.org/10.1090/S0894-0347-10-00670-3

Tod10b_ Generating functions of stable pair invariants via wall-crossings in derived categories, in New developments in algebraic geometry, integrable systems and mirror symmetry (RIMS, Kyoto, 2008), 389-434, Adv. Stud. Pure Math. 59, Math. Soc. Japan, Tokyo, 2010.

Tod12_, Stability conditions and curve counting invariants on Calabi-Yau 3-folds, Kyoto Journal of Mathematics 52 (2012), 1-50. http://dx.doi.org/10.1215/21562261-1503745

Tod13 Y. Toda, Gepner point and strong Bogomolov-Gieseker inequality for quintic 3-folds, to appear in Professor Kawamata's 60th birthday volume, arXiv:1305.0345.

Ued12 K. Ueda, Hyperplane sections and stable derived categories, arXiv:1207.1167.

Wal05 J. Walcher, Stability of Landau-Ginzburg branes, J. Math. Phys. 46 (2005). http://dx.doi. org/10.1063/1.2007590

Yukinobu Toda yukinobu.toda@ipmu.jp

Kavli Institute for the Physics and Mathematics of the Universe, University of Tokyo, 5-1-5 Kashiwanoha, Kashiwa, 277-8583, Japan 